SAND2005-XXXX

Printed XXXX 2005

\title{
Adhesive Joint and Composites Modeling in SIERRA
}

\author{
Daniel C. Hammerand, Robert S. Chambers, Arthur A. Brown, James W. Foulk, \\ Douglas B. Adolf and Yuki Ohashi \\ Sandia National Laboratories \\ P.O. Box 5800 \\ Albuquerque, NM 87185-0893
}

\begin{abstract}
Polymers and fiber-reinforced polymer matrix composites play an important role in many Defense Program applications. Recently an advanced nonlinear viscoelastic model for polymers has been developed and incorporated into ADAGIO, Sandia's SIERRA-based quasi-static analysis code. Standard linear elastic shell and continuum models for fiber-reinforced polymer-matrix composites have also been added to ADAGIO. This report details the use of these models for advanced adhesive joint and composites simulations carried out as part of an Advanced Simulation and Computing Advanced Deployment (ASC AD) project. More specifically, the thermo-mechanical response of an adhesive joint when loaded during repeated thermal cycling is simulated, the response of some composite rings under internal pressurization is calculated, and the performance of a composite container subjected to internal pressurization, thermal loading, and distributed mechanical loading is determined. Finally, general comparisons between the continuum and shell element approaches for modeling composites using ADAGIO are given.
\end{abstract}




\section{Contents}

1 Introduction $\quad 12$

2 Adhesively Bonded Joint 14

2.1 Problem Definition . . . . . . . . . . . . . . . . . . . . . 14

2.2 Thermo-mechanical Response . . . . . . . . . . . . . . . . . 16

3 Isotropic and Composite Circular Rings Under Internal Pressure 22

3.1 Composite Modeling Overview . . . . . . . . . . . . . . . 22

3.2 Ring A Problem Definition . . . . . . . . . . . . . . . . . . 24

3.3 Ring A Results . . . . . . . . . . . . . . . . . . . 25

3.4 Ring B Problem Definition . . . . . . . . . . . . . 28

3.5 Ring B Results . . . . . . . . . . . . . . . . . . . . . 31

4 Composite Containers $\quad 66$

4.1 Problem Definition . . . . . . . . . . . . . . . 66

4.2 Response of Composite Containers to Pressure, Thermal, and Mechanical Loadings . . . . . . . . . . . . . . . . . . . . . 69

5 Comparison of Shell and Continuum Element Approaches for Composite Modeling $\quad 89$

$\begin{array}{lll}6 & \text { Summary } & 93\end{array}$

$\begin{array}{lll}7 & \text { References } & 94\end{array}$

8 Appendix A: ADAGIO Input Deck for the Adhesive Joint Problem 95

9 Appendix B: ADAGIO Input Deck for [30/60/50/10] Ring B Modeled Using Continuum Elements 109

10 Appendix C: ADAGIO Input Deck for [30/60/50/10] Ring B Modeled Using Shell Elements 


\section{List of Figures}

2.1 Schematic of bonded cylinder geometry. . . . . . . . . . . . 15

2.2 View of finite element mesh at end seal. . . . . . . . . . . . . . 17

2.3 Maximum principal stresses $(\mathrm{Pa})$ in adhesive at $T=20{ }^{\circ} \mathrm{C}$ prior to sealing. 18

2.4 von Mises stresses $(\mathrm{Pa})$ in outer cylinder at $T=20{ }^{\circ} \mathrm{C}$ prior to sealing. $\quad 18$

2.5 von Mises element stresses (Pa) after application of pre-load. . . . . . 19

2.6 von Mises stresses (Pa) in seal at $T=70{ }^{\circ} \mathrm{C}$ under $30000 \mathrm{lb}$ load. . . . 20

2.7 von Mises stresses (Pa) in seal at $T=-55{ }^{\circ} \mathrm{C}$ under $5000 \mathrm{lb}$ load. . . . . 20

2.8 Maximum principal stresses in adhesive at $T=-55{ }^{\circ} \mathrm{C}$ under $5000 \mathrm{lb}$ load. 21

3.1 Ring A geometry. . . . . . . . . . . . . . . . . . . . . 24

3.2 Deformed [0/90/90/0] composite Ring A (100X mag. of displacements). 26

3.3 Deformed [30/30/30/30] composite Ring A (100X mag. of displacements). 27

3.4 Undeformed and deformed side views of [30/30/30/30] composite Ring A (100X mag. of displacements). . . . . . . . . . . . . . 28

3.5 Deformed [10/50/60/30] composite Ring A (100X mag. of displacements). 29

3.6 Deformed side view of $[10 / 50 / 60 / 30]$ composite Ring A (100X mag. of displacements). . . . . . . . . . . . . . . . . . . . 29

3.7 Deformed isotropic Ring B continuum model showing 1 element in circumferential direction. Deformations magnified by 1000. . . . . . . 36

3.8 Deformed isotropic Ring B shell model showing 1 element in circumferential direction. Deformations magnified by 1000. . . . . . . . . 37

3.9 Deformed [0/90/90/0] Ring B continuum model showing 1 element in circumferential direction. Deformations magnified by 1000 . . . . . 40

3.10 Deformed [0/90/90/0] Ring B shell model showing 1 element in circumferential direction. Deformations magnified by 1000 . . . . . . . . . 41

3.11 Deformed 0.1" high [30/45/45/30] Ring B continuum model showing 1 element in circumferential direction. Deformations magnified by 1000 .

3.12 Deformed 0.1" high [30/45/45/30] Ring B shell model showing 1 element in circumferential direction. Deformations magnified by 1000. . . . . . 45

3.13 Deformed 0.01" high [30/45/45/30] Ring B continuum model showing 1 element in circumferential direction. Deformations magnified by 1000.48 
3.14 Stress distribution for deformed 0.01" high [30/45/45/30] Ring B continuum model: (a) $\sigma_{X X}=\sigma_{r r}$; (b) $\sigma_{Y Y}=\sigma_{z z}$; (c) $\sigma_{Z Z}=\sigma_{\theta \theta}$. Deformations magnified by $1000 . \ldots \ldots \ldots \ldots$

3.15 Stress distribution for deformed 0.01" high [30/45/45/30] Ring B continuum model: (a) $\sigma_{X Y}=\sigma_{z r}$; (b) $\sigma_{Y Z}=-\sigma_{\theta z}$; (c) $\sigma_{Z X}=-\sigma_{r \theta}$. Deformations magnified by 1000. . . . . . . . . . . . 50

3.16 Deformed 0.01" high [30/45/45/30] Ring B shell model showing 1 element in circumferential direction. Deformations magnified by 1000 . .

3.17 Force resultants distribution for deformed 0.01" high [30/45/45/30] Ring B shell model: (a) $N_{11}=N_{\theta \theta}$; (b) $N_{22}=N_{z z}$; (c) $N_{12}=N_{\theta z}$. Deformations magnified by 1000. . . . . . . . . . . 52

3.18 Force resultants distribution for deformed 0.01" high [30/45/45/30] Ring B shell model: (a) $N_{23}=N_{z r}$; (b) $N_{31}=N_{r \theta}$. Deformations magnified by $1000 \ldots \ldots \ldots \ldots \ldots$

3.19 Force-couple resultants distribution for deformed 0.01" high [30/45/45/30] Ring B shell model: (a) $M_{11}=M_{\theta \theta}$; (b) $M_{22}=M_{z z}$; (c) $M_{12}=M_{\theta z}$. Deformations magnified by $1000 \ldots \ldots$. . . . . . . . . 54

3.20 Deformed [30/-30/30/-30] Ring B continuum model showing 1 element in circumferential direction. Deformations magnified by 1000 . . . . . 57

3.21 Deformed [30/-30/30/-30] Ring B shell model showing 1 element in circumferential direction. Deformations magnified by 1000 . . . . . 58

3.22 Inplane membrane strain distribution for deformed [30/-30/30/-30] Ring B shell model: (a) $e_{11}=e_{\theta \theta}$; (b) $e_{22}=e_{z z}$; (c) $2 e_{12}=2 e_{\theta z}$. Deformations magnified by $1000 \ldots \ldots \ldots \ldots \ldots \ldots$

3.23 Transverse shear strain distribution for deformed [30/-30/30/-30] Ring B shell model: (a) $2 e_{23}=2 e_{z r}$; (b) $2 e_{31}=2 e_{r}$. Deformations magnified by $1000 \ldots \ldots \ldots \ldots \ldots \ldots \ldots \ldots$

3.24 Bending strain distribution for deformed [30/-30/30/-30] Ring B shell model: (a) $\kappa_{11}=\kappa_{\theta \theta}$; (b) $\kappa_{22}=\kappa_{z z}$; (c) $2 \kappa_{12}=2 \kappa_{\theta z}$. Deformations magnified by $1000 \ldots \ldots \ldots \ldots \ldots \ldots$

3.25 Deformed [30/60/50/10] Ring B continuum model showing 1 element in circumferential direction. Deformations magnified by 1000 . . . . . 
3.26 Deformed [30/60/50/10] Ring B shell model showing 1 element in circumferential direction. Deformations magnified by 1000. . . . . . 65

4.1 Composite container geometry. . . . . . . . . . . . 67

4.2 Cross sectional view of composite container with [0/90/90/0] lay-up modeled using only continuum elements with 10X mag. of final displacements. ........................... 71

4.3 Cross sectional view of composite container with [0/90/90/0] lay-up modeled using only primarily shell elements with 10X mag. of final displacements. . . . . . . . . . . . . . . . . 72

4.4 Cross sectional view of composite container with [30/45/45/30] layup modeled using only continuum elements with 10X mag. of final displacements. . . . . . . . . . . . . . . . . 72

4.5 Cross sectional view of composite container with [30/-30/30/-30] layup modeled using only continuum elements with 10X mag. of final displacements. . . . . . . . . . . . . . . 73

4.6 von Mises stresses due to internal pressurization, thermal loading, and mechanical loading for the $[0 / 90 / 90 / 0]$ container modeled using only continuum elements: (a) layer 1 (outermost layer); (b) layer 2; (c) layer 3 ; (d) layer 4 (innermost layer). . . . . . . . . . . . . .

4.7 von Mises stresses due to internal pressurization, thermal loading, and mechanical loading for the [30/45/45/30] container modeled using only continuum elements: (a) layer 1 (outermost layer); (b) layer 2; (c) layer 3 ; (d) layer 4 (innermost layer). . . . . . . . . . . . . .

4.8 von Mises stresses due to internal pressurization, thermal loading, and mechanical loading for the [30/-30/30/-30] container modeled using only continuum elements: (a) layer 1 (outermost layer); (b) layer 2; (c) layer 3 ; (d) layer 4 (innermost layer). . . . . . . . . . . . . . 76

4.9 Radial stresses due to internal pressurization for the $[0 / 90 / 90 / 0]$ container modeled using only continuum elements: (a) layer 1 (outermost layer); (b) layer 2; (c) layer 3; (d) layer 4 (innermost layer). . . . . . 
4.10 Axial stresses due to internal pressurization for the $[0 / 90 / 90 / 0]$ container modeled using only continuum elements: (a) layer 1 (outermost layer); (b) layer 2; (c) layer 3; (d) layer 4 (innermost layer). . . . . . . 78

4.11 Circumferential stresses due to internal pressurization for the $[0 / 90 / 90 / 0]$ container modeled using only continuum elements: (a) layer 1 (outermost layer); (b) layer 2; (c) layer 3; (d) layer 4 (innermost layer). . . .

4.12 Radial-axial shear stresses due to internal pressurization for the [0/90/90/0] container modeled using only continuum elements: (a) layer 1 (outermost layer); (b) layer 2; (c) layer 3; (d) layer 4 (innermost layer). . . . 80

4.13 Negative of axial-circumferential shear stresses due to internal pressurization for the $[0 / 90 / 90 / 0]$ container modeled using only continuum elements: (a) layer 1 (outermost layer); (b) layer 2; (c) layer 3; (d) layer 4 (innermost layer). . . . . . . . . . . . . . . . . . . 81

4.14 Negative of circumferential-radial shear stresses due to internal pressurization for the $[0 / 90 / 90 / 0]$ container modeled using only continuum elements: (a) layer 1 (outermost layer); (b) layer 2; (c) layer 3; (d) layer 4 (innermost layer). . . . . . . . . . . . . . . . . . . 82

4.15 Radial stresses due to internal pressurization for the $[0 / 90 / 90 / 0]$ container modeled using primarily shell elements: (a) layer 1 (outermost layer); (b) layer 2; (c) layer 3; (d) layer 4 (innermost layer). . . . . . . 83

4.16 Axial stresses due to internal pressurization for the $[0 / 90 / 90 / 0]$ container modeled using primarily shell elements: (a) layer 1 (outermost layer); (b) layer 2; (c) layer 3; (d) layer 4 (innermost layer). . . . . . .

4.17 Circumferential stresses due to internal pressurization for the $[0 / 90 / 90 / 0]$ container modeled using primarily shell elements: (a) layer 1 (outermost layer); (b) layer 2 ; (c) layer 3 ; (d) layer 4 (innermost layer). . . . . . .

4.18 Radial-axial shear stresses due to internal pressurization for the [0/90/90/0] container modeled using primarily shell elements: (a) layer 1 (outermost layer); (b) layer 2; (c) layer 3; (d) layer 4 (innermost layer). . . . . . . 86 
4.19 Negative of axial-circumferential shear stresses due to internal pressurization for the $[0 / 90 / 90 / 0]$ container modeled using primarily shell elements: (a) layer 1 (outermost layer); (b) layer 2; (c) layer 3; (d) layer 4 (innermost layer). . . . . . . . . . . . . . . . 87

4.20 Negative of circumferential-radial shear stresses due to internal pressurization for the $[0 / 90 / 90 / 0]$ container modeled using primarily shell elements: (a) layer 1 (outermost layer); (b) layer 2; (c) layer 3; (d) layer 4 (innermost layer).

\section{List of Tables}

3.1 Radial displacement at middle of inside surface for isotropic Ring B. . 34

3.2 Stress $\sigma_{X X}=\sigma_{r r}$ at ring center for isotropic Ring B. . . . . . . . . . 34

3.3 Stress $\sigma_{Y Y}=\sigma_{z z}$ at ring center for isotropic Ring B. . . . . . . . . . . 34

3.4 Stress $\sigma_{Z Z}=\sigma_{\theta \theta}$ at ring center for isotropic Ring B. . . . . . . . . . . 34

3.5 Stress $\sigma_{X Y}=\sigma_{z r}$ at ring center for isotropic Ring B. . . . . . . . . 35

3.6 Stress $\sigma_{Y Z}=-\sigma_{\theta z}$ at ring center for isotropic Ring B. . . . . . . . 35

3.7 Stress $\sigma_{Z X}=-\sigma_{r \theta}$ at ring center for isotropic Ring B. . . . . . . . . 35

3.8 Computational cost for isotropic Ring B. . . . . . . . . . . . . . . 35

3.9 Radial displacement at middle of inside surface for [0/90/90/0] Ring B. 38

3.10 Stress $\sigma_{X X}=\sigma_{r r}$ at ring center for $[0 / 90 / 90 / 0]$ Ring B. . . . . . . . 38

3.11 Stress $\sigma_{Y Y}=\sigma_{z z}$ at ring center for $[0 / 90 / 90 / 0]$ Ring B. . . . . . . . . 38

3.12 Stress $\sigma_{Z Z}=\sigma_{\theta \theta}$ at ring center for $[0 / 90 / 90 / 0]$ Ring B. . . . . . . . 38

3.13 Stress $\sigma_{X Y}=\sigma_{z r}$ at ring center for $[0 / 90 / 90 / 0]$ Ring B. . . . . . . . 38

3.14 Stress $\sigma_{Y Z}=-\sigma_{\theta z}$ at ring center for $[0 / 90 / 90 / 0]$ Ring B. . . . . . . 39

3.15 Stress $\sigma_{Z X}=-\sigma_{r \theta}$ at ring center for [0/90/90/0] Ring B. . . . . . . . 39

3.16 Computational cost for $[0 / 90 / 90 / 0]$ Ring B. . . . . . . . . . . . 39

3.17 Radial displacement at middle of inside surface for 0.1" high [30/45/30/30]

Ring B. . . . . . . . . . . . . . . . . . . . 42

3.18 Stress $\sigma_{X X}=\sigma_{r r}$ at ring center for 0.1" high [30/45/30/30] Ring B. . 42

3.19 Stress $\sigma_{Y Y}=\sigma_{z z}$ at ring center for 0.1" high [30/45/30/30] Ring B. . 42

3.20 Stress $\sigma_{Z Z}=\sigma_{\theta \theta}$ at ring center for 0.1” high [30/45/30/30] Ring B. . . 42 
3.21 Stress $\sigma_{X Y}=\sigma_{z r}$ at ring center for 0.1" high [30/45/30/30] Ring B. . 42

3.22 Stress $\sigma_{Y Z}=-\sigma_{\theta z}$ at ring center for 0.1" high [30/45/30/30] Ring B. 43

3.23 Stress $\sigma_{Z X}=-\sigma_{r \theta}$ at ring center for 0.1" high [30/45/30/30] Ring B. 43

3.24 Computational cost for 0.1" high [30/45/30/30] Ring B. . . . . . . . . 43

3.25 Radial Displacement at middle of inside surface for 0.01" high [30/45/30/30]

Ring B. . . . . . . . . . . . . . . . . . 46

3.26 Stress $\sigma_{X X}=\sigma_{r r}$ (average over layer) for 0.01" high [30/45/30/30] Ring

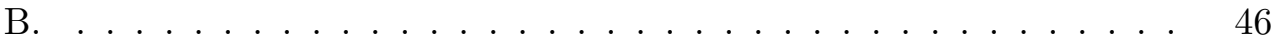

3.27 Stress $\sigma_{Y Y}=\sigma_{z z}$ (average over layer) for 0.01" high [30/45/30/30] Ring

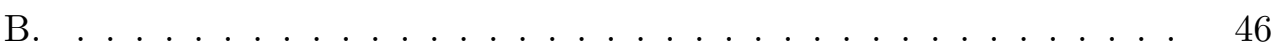

3.28 Stress $\sigma_{Z Z}=\sigma_{\theta \theta}$ (average over layer) for 0.01" high [30/45/30/30] Ring

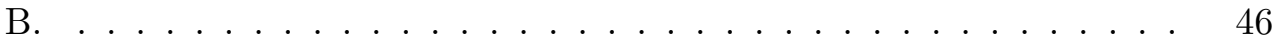

3.29 Stress $\sigma_{X Y}=\sigma_{z r}$ (average over layer) for 0.01" high [30/45/30/30] Ring

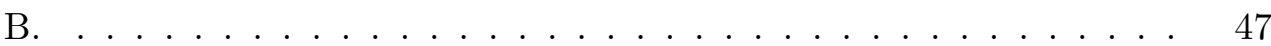

3.30 Stress $\sigma_{Y Z}=-\sigma_{\theta z}$ (average over layer) for 0.01" high [30/45/30/30]

Ring B. . . . . . . . . . . . . . . . . . 47

3.31 Stress $\sigma_{Z X}=-\sigma_{r \theta}$ (average over layer) for 0.01 " high [30/45/30/30]

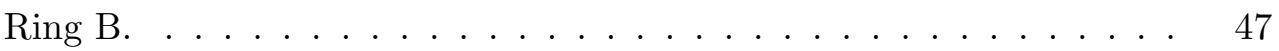

3.32 Computational cost for 0.01" high [30/45/30/30] Ring B. . . . . . . 47

3.33 Radial displacement at middle of inside surface for [30/-30/30/-30] Ring

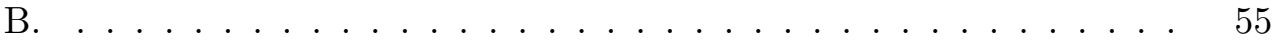

3.34 Stress $\sigma_{X X}=\sigma_{r r}$ at ring center for $[30 /-30 / 30 /-30]$ Ring B. . . . . . 55

3.35 Stress $\sigma_{Y Y}=\sigma_{z z}$ at ring center for [30/-30/30/-30] Ring B. . . . . . 55

3.36 Stress $\sigma_{Z Z}=\sigma_{\theta \theta}$ at ring center for [30/-30/30/-30] Ring B. . . . . . 55

3.37 Stress $\sigma_{X Y}=\sigma_{z r}$ at ring center for [30/-30/30/-30] Ring B. . . . . . . 55

3.38 Stress $\sigma_{Y Z}=-\sigma_{\theta z}$ at ring center for $[30 /-30 / 30 /-30]$ Ring B. . . . . . 56

3.39 Stress $\sigma_{Z X}=-\sigma_{r \theta}$ at ring center for [30/-30/30/-30] Ring B. . . . . . 56

3.40 Computational cost for $[30 /-30 / 30 /-30]$ Ring B . . . . . . . . . . . . 56

3.41 Radial Displacement at middle of inside surface for [30/60/50/10] Ring

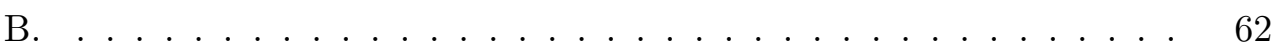

3.42 Stress $\sigma_{X X}=\sigma_{r r}$ at ring center for [30/60/50/10] Ring B. . . . . . . 62

3.43 Stress $\sigma_{Y Y}=\sigma_{z z}$ at ring center for $[30 / 60 / 50 / 10]$ Ring B. . . . . . . . 62 
3.44 Stress $\sigma_{Z Z}=\sigma_{\theta \theta}$ at ring center for [30/60/50/10] Ring B. . . . . . . . 62

3.45 Stress $\sigma_{X Y}=\sigma_{z r}$ at ring center for [30/60/50/10] Ring B. . . . . . . . 62

3.46 Stress $\sigma_{Y Z}=-\sigma_{\theta z}$ at ring center for [30/60/50/10] Ring B. . . . . . . 63

3.47 Stress $\sigma_{Z X}=-\sigma_{r \theta}$ at ring center for [30/60/50/10] Ring B. . . . . . . 63

3.48 Computational cost for $[30 / 60 / 50 / 10]$ Ring B. . . . . . . . . . . . 63

4.1 CPU usage for composite container analyses. . . . . . . . . . . 70 


\section{Introduction}

Polymers play an important role in many different Defense Program applications. They provide structural integrity, environmental damping, moisture and voltage isolation and are employed as encapsulants, adhesives and coatings in a variety of weapon components. Polymers are attractive for their low cost, ease of manufacturing, and the ability of their material properties to be tailored through the addition of fillers. However, their thermo-mechanical behavior can be extremely complex. Since polymers are viscoelastic materials, they undergo a glass transition that radically alters the perceived physical properties of the material (varying from the rubbery state above $\mathrm{Tg}$ to the glassy state below). Stress and volume relaxation rates are quite sensitive to temperature, and glassy materials can actually yield under loading. The richness of the material behavior makes it important to understand how these materials react to their operational environment. This understanding can aid in the design process, helping to avoid cohesive cracking within the polymer or delamination at key material interfaces.

For this ASC AD project, we are specifically interested in being able to analyze two kinds of material behavior:

1. the thermo-mechanical response of an adhesive joint when loaded during repeated thermal cycling, and

2. the performance of an anisotropic elastic (glassy) structure subjected to internal pressurization, thermal loading, and distributed mechanical loading

Item one calls for a nonlinear viscoelastic model of the adhesive to predict the stresses and strains generated during thermal cycling and loading through glass transition. The polymer constitutive model was developed in past years through collaboration between the Engineering and Materials Sciences Centers and has been implemented in the ADAGIO finite element code. The problem we will be analyzing consists of two concentric metal cylinders bonded together with an adhesive layer. The outer cylinder is shorter and serves as a stiffener that is sealed at both ends to the longer inner cylinder (case). The combined structure is then axially loaded and thermally cycled. Of particular interest is the design and loading of the end seal. This problem will 
be among the first major applications to benefit from nonlinear viscoelastic modeling. Moreover, it will afford critical opportunities to evaluate computational performance in a large problem setting while also introducing the DP customer to the benefits of being able to make higher fidelity predictions.

The second item involves new material models and associated code enhancements to accommodate orthotropic/anisotropic elastic materials with thermal strains. For many years, Sandia has had to resort to commercial software (ABAQUS) in order to perform such analyses. Recent and ongoing ADAGIO code development has provided both orthotropic hex elements and anisotropic shell elements complete with thermal strains giving us new in-house capabilities to fulfill this need. A general capability for initializing material orientations has been incorporated into ADAGIO as well. The problem chosen for study is a composite structural container under pressure, thermal, and mechanical loadings. Preliminary results from a purely continuum element approach and a combined shell/continuum element approach will be given. However, to better understand the differences between shell and continuum modeling approaches, comparisons will be made first for rings under internal pressure. The importance of using true cyclic periodic boundary conditions in place of axisymmetric cyclic boundary conditions for composite structures also will be demonstrated. Moreover, general observations will be made about the differences between shell and continuum approaches for modeling composite structures. 


\section{Adhesively Bonded Joint}

The nonlinear viscoelastic (NLVE) analysis of adhesives lends itself to staged calculations where the epoxy first undergoes solidification (curing) at elevated temperatures followed by a thermal cool-down and any subsequent structural modifications and loading. Although a theory for NLVE curing exists, the requisite material characterization and validation are not yet complete and the SIERRA infrastructure is still under construction (e.g., material model library and material interface wrapper for incompressibility). Hence, chemistry (e.g., cure shrinkage effects) is neglected in all subsequent computations.

\subsection{Problem Definition}

The proposed problem consists of two concentric cylinders that are adhesively bonded together and sealed at the ends. A schematic of the geometry is shown in Figure 1. During manufacturing, the outer cylinder is glued to the inner cylinder at an elevated temperature. The joined cylinders then are cooled to room temperature where the end of the adhesive joint is sealed metallically. Following this, the inner cylinder is axially loaded by 18000 lbs $(80067 \mathrm{~N})$ producing a nominal end stress of about 8127 psi $(56 \mathrm{MPa})$. The entire assembly is then thermally cycled between $70{ }^{\circ} \mathrm{C}$ and $55^{\circ} \mathrm{C}$ at $3{ }^{\circ} \mathrm{C} / \mathrm{min}$. During the temperature ramps the axial loading is linearly increased to $30000 \mathrm{lbs}$ at the upper temperature and decreased to $5000 \mathrm{lbs}$ at $55^{\circ} \mathrm{C}$. At $70{ }^{\circ} \mathrm{C}$, the temperature is held constant for 2 hours.

For viscoelastic materials, the analysis must begin from a well-defined reference (equilibrium) state. In a curing epoxy, this naturally would correspond to the elevated cure temperature where the solidification reaction occurs as the material transitions from a liquid to a solid. However, since polymerization is being neglected, the analysis was initiated from the reference temperature (Tref $=85{ }^{\circ} \mathrm{C}$ ) defined for the fully cured material as Tref $=\mathrm{Tg}+10{ }^{\circ} \mathrm{C}$. Although this was somewhat higher than the actual curing temperature of $60{ }^{\circ} \mathrm{C}$, it produces a rubbery thermal shrinkage in the direction of what would have been realized by cure shrinkage during polymerization. The temperature was decreased at $1{ }^{\circ} \mathrm{C} / \mathrm{min}$ from $85{ }^{\circ} \mathrm{C}$ to $60{ }^{\circ} \mathrm{C}$ (approximate cure temperature) and then cooled at $5{ }^{\circ} \mathrm{C} / \mathrm{min}$ to $20{ }^{\circ} \mathrm{C}$ for the end sealing operation. 


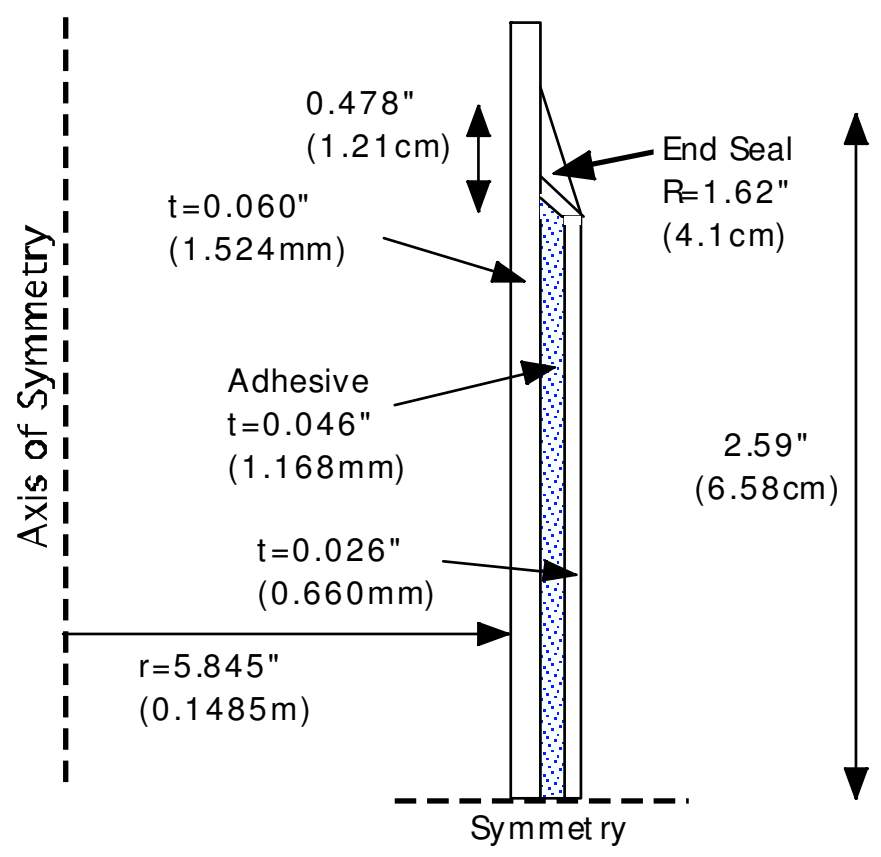

Figure 2.1: Schematic of bonded cylinder geometry.

Notice that during the adhesive bonding and initial cool-down stage, the end seal is not actually present. Geometrically, it is undesirable to birth finite elements onto a deformed geometry. Hence, for modeling purposes only, the end seal was included in the initial mesh, but it was assigned a low modulus. This allowed the material to deform freely under low load during the initial cooling and then structurally enter the problem with a stiffer modulus in the next stage of the analysis after the seal is formally applied at room temperature.

The analysis was performed with SIERRA. Since this is a quasi-static response, ADAGIO was the code of choice. However, to execute the staged analyses allowing the material properties of the metallic seal to be initially low and then increased partway through the analysis, the TEMPO code had to be employed linking two separate ADAGIO analyses. The annotated material model input deck is included in Appendix A. Note that fixed contact was used to tie the corners of the mesh at the top end under the metallic seal. Since the actual geometry of the outer cylinder tapers into the inner cylinder at the seal end, a fixed contact condition was assumed at this interface realizing that adhesive was likely to fill the void and bond the two surfaces 
together.

The problem was meshed as a 90-degree sector of the cylinders with symmetry boundary conditions applied along the bottom end of the mesh perpendicular to the axis as shown in Figure 2.1. The total model consisted of 68490 elements and 81328 nodes. Although the mesh size is small by comparison to many models, it must be remembered that the computational expense involved in nonlinear viscoelastic analyses is much greater than elasticity or plasticity. Moreover, the solution required 460 time steps to track the staged solution history. This is more than sufficient to shake out the workings of SIERRA code and demonstrate capability to a customer. A detailed view of the finite element mesh in the vicinity of the end seal is shown in Figure 2.2. Here all three materials are visible. The inner and outer cylinders are made of 6061T6 aluminum and the metallic seal is made from an 1100 aluminum alloy. The aluminum cylinders and seal are assumed to have the same properties: Young's modulus=69 GPa, Poisson's ratio $=0.33$, and $\mathrm{CTE}=23.4 \mathrm{ppm} / \mathrm{C}$. A definition of the nonlinear viscoelastic material properties for the adhesive is provided through the ADAGIO input deck in APPENDIX A. Because of its length, it is not reproduced here. The analysis was performed on "Rogue", a 407-node Linux cluster. Rogue is divided into two parts. Jobs run in the "Serial" queue are performed using $2.4 \mathrm{GHz}$ Xeon compute nodes, whereas jobs performed in the "Scico" queue are run using $3.06 \mathrm{GHz}$ Xeon compute nodes. Note that parallel jobs can be run in either queue. Using 16 processors in the Scico queue, the execution time was just over 10 hours.

\subsection{Thermo-mechanical Response}

In the first stage of the analysis, residual stresses are generated as the adhesively bonded cylinders are cooled to $20{ }^{\circ} \mathrm{C}$. These stresses arise from the mismatch between the thermal strains in the aluminum and adhesive. Because the adhesive originally is at a temperature higher than its glass transition temperature $(\mathrm{Tg})$, it first exhibits a rubbery response and then becomes glassy below Tg. Figure 2.3 contains a color plot of the maximum principal stresses in the adhesive at $\mathrm{T}=20^{\circ} \mathrm{C}$ after cooling just prior to adding the metallic seal. The adhesive shear stresses are an order of magnitude lower. Figure 2.4 illustrates the corresponding Von Mises stress in the outer aluminum cylinder. The stresses in the inner cylinder are less than half the magnitude of those 


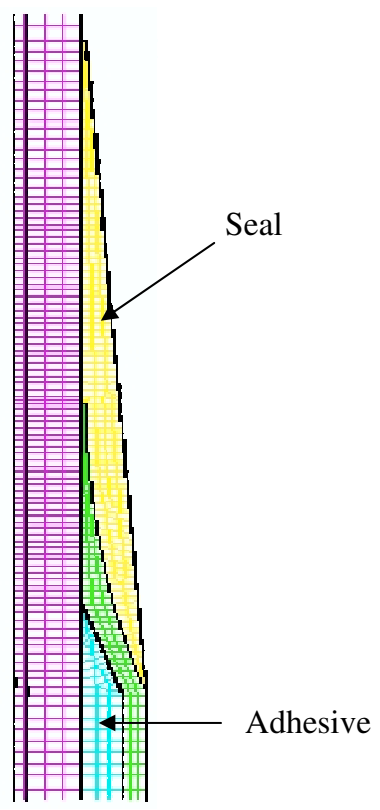

Figure 2.2: View of finite element mesh at end seal.

in Figure 2.4.

No attempt was made to analyze any manufacturing stresses induced by the end sealing operation at room temperature. Rather the Young's modulus of the metallic seal was increased from the fictitiously low value of $1 \mathrm{MPa}$ to $69 \mathrm{GPa}$ effectively giving birth to the joint for the remainder of the analyses. This provided a realistic stiffness to resist subsequent loading. Note also there is the implicit assumption that the interface between the inner and outer cylinders (underneath the metallic seal) is adhesively bonded together. This is achieved by a fixed contact.

Once the end seal is in place the inner cylinder is axial pre-loaded in tension under 18000 lbs $(80067 \mathrm{~N})$. A plot of the Von Mises element stresses is shown in Figure 2.5 just after application of the pre-load. The magnitude of the maximum stress in the inner cylinder, outer cylinder, and metallic seal are $61 \mathrm{MPa}(8.85 \mathrm{ksi}), 69 \mathrm{MPa}$ (10 ksi), and $51 \mathrm{MPa}(7.4 \mathrm{ksi})$, respectively. The highest stress in the metallic seal during the entire thermo- mechanical history was recorded at the $70{ }^{\circ} \mathrm{C}$ temperature where the axial load was increased to 30000 lbs. A plot of these Von Mises stresses is provided in Figure 2.6. Here the maximum stress is seen to reach $85 \mathrm{MPa}$ and is located along the thin top end of the seal. The stress in the area of the cylinder overlap 


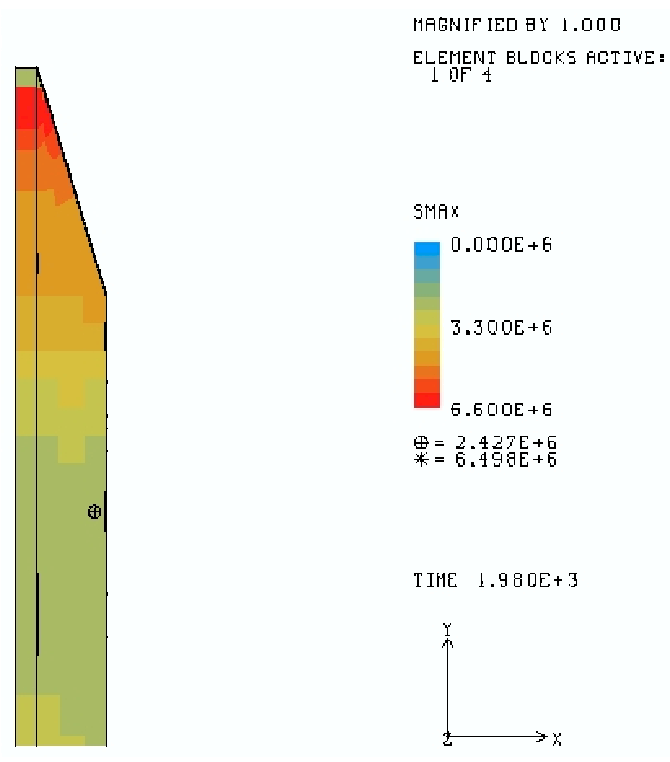

Figure 2.3: Maximum principal stresses $(\mathrm{Pa})$ in adhesive at $T=20{ }^{\circ} \mathrm{C}$ prior to sealing.

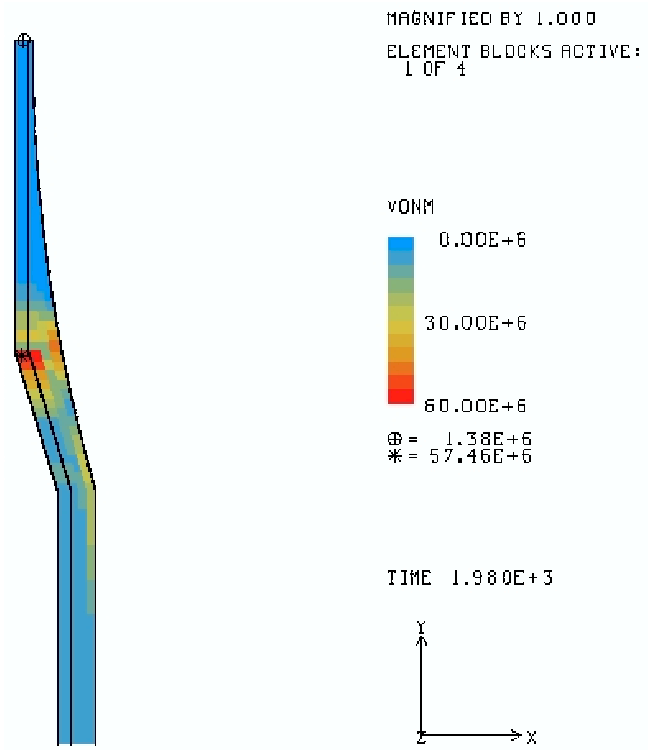

Figure 2.4: von Mises stresses $(\mathrm{Pa})$ in outer cylinder at $T=20{ }^{\circ} \mathrm{C}$ prior to sealing. 


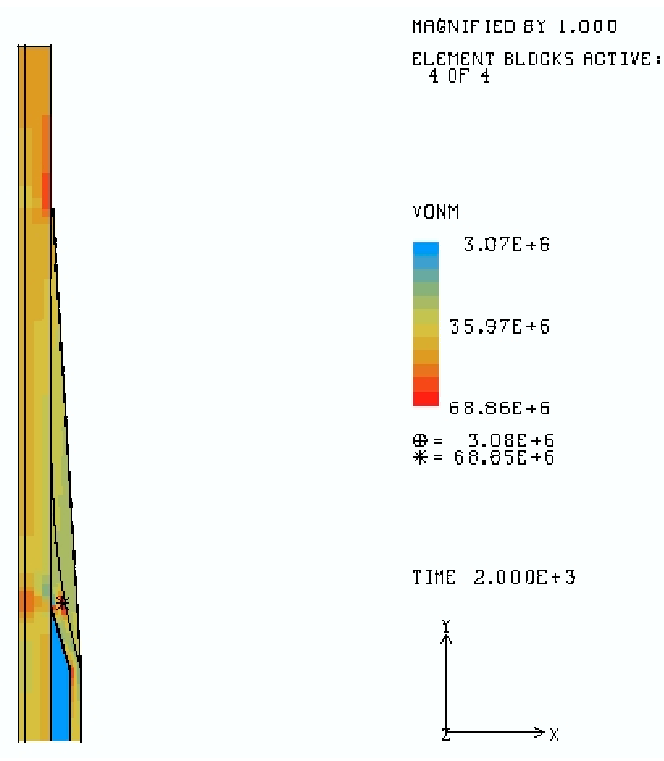

Figure 2.5: von Mises element stresses (Pa) after application of pre-load.

is between 45 and $55 \mathrm{MPa}$. The seal stresses at the lower temperature $\left(\mathrm{T}=-55{ }^{\circ} \mathrm{C}\right)$ are actually reduced somewhat due to the fact that the axial pre-load is only $5000 \mathrm{lbs}$. These stresses are plotted in Figure 2.7. However, the location of the maximum stress does shift towards the opposite end of the seal. The maximum principal stresses in the adhesive are greatest at the lower temperature due to the increased mismatch in thermal strains under the geometric constraint between cylinders. These stresses are plotted in Figure 2.8. 


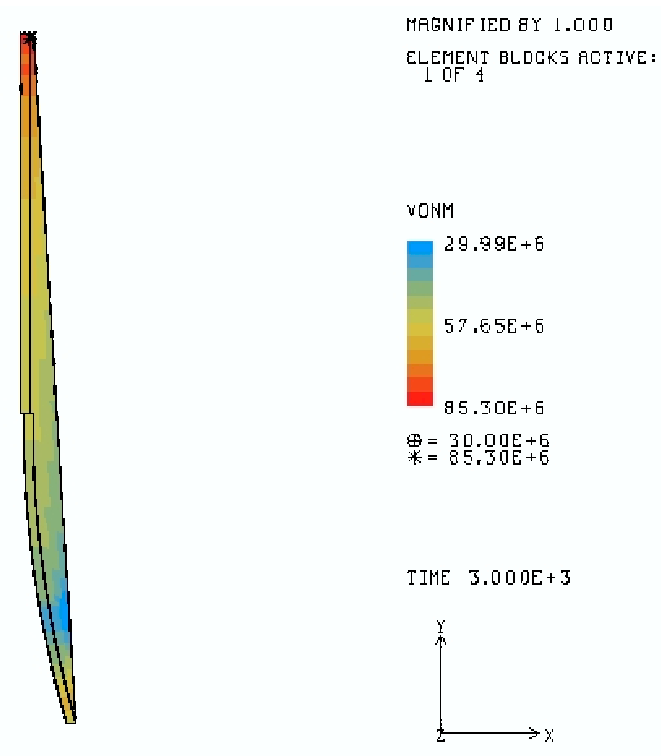

Figure 2.6: von Mises stresses $(\mathrm{Pa})$ in seal at $T=70{ }^{\circ} \mathrm{C}$ under $30000 \mathrm{lb}$ load.

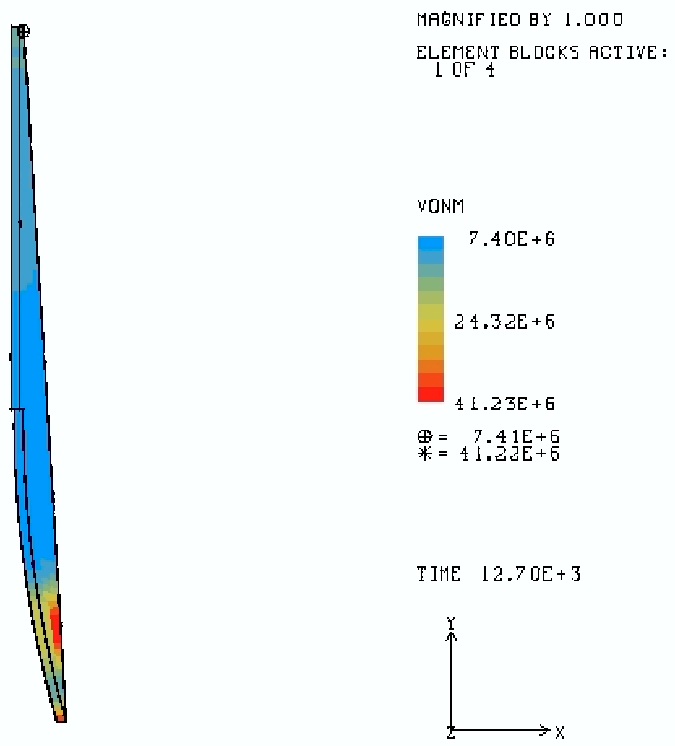

Figure 2.7: von Mises stresses $(\mathrm{Pa})$ in seal at $T=-55{ }^{\circ} \mathrm{C}$ under $5000 \mathrm{lb}$ load. 


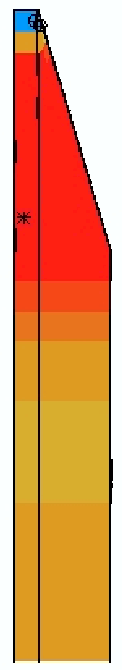

MAGNIF IED QY 1.000

ELEMENT BLDGKS AGTIVE:

SMAX

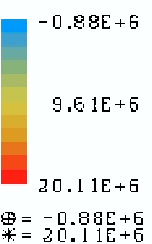

T IME $\quad 12.70 \mathrm{DE}+3$

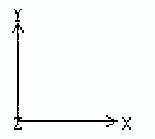

Figure 2.8: Maximum principal stresses in adhesive at $T=-55^{\circ} \mathrm{C}$ under $5000 \mathrm{lb}$ load. 


\section{Isotropic and Composite Circular Rings Under Internal Pressure}

\subsection{Composite Modeling Overview}

Laminated fiber-reinforced composite structures can be classified as plate or shells in almost all cases. Recall that plates refer to flat structures which are thin in their thickness direction compared to their inplane dimensions, whereas shells refer to similar structures which are curved. Due to this small thickness, certain kinematic assumptions can usually be applied in analyzing such structures. If a plate or shell is thin enough, the usual Kirchhoff bending constraints of normals to the midplane remaining straight and normal to the midplane under the application of load can be applied. That is, if thin enough, the plate or shell will not exhibit any transverse shear response. However, if a structure is sufficiently thick, this condition needs to be relaxed to allow the transverse shear response of the structure to be captured. In such cases, Mindlin theory is typically applied. In a majority of cases, using a plate/shell theory in modeling a composite structure is usually sufficient. However, using such a theory is not a requirement. That is, such composites can be analyzed using an appropriate continuum approach. Such a continuum approach does not involve enforcing any kinematic conditions likely to result for a given structure a priori beyond the usual selection of a suitable strain measure.

Regardless of whether a shell or continuum approach is used, it is usually necessary to use a numerical method to determine the structural response of a composite structure for most cases beyond a simple few for which analytical results can be determined. In structural mechanics, the favored approach is, of course, finite element analysis. The pertinent questions are then when can shell elements be used accurately and efficiently? and when will it be necessary to use continuum elements? ADAGIO and PRESTO already have the necessary constitutive laws for models using either approach. ${ }^{1}$ The continuum material law that can be used for an individual layer is given by the inverse of Eq. (1.1) in Reference 1, whereas the laminate material model that can be used for an entire lay-up is given by Eqs. (1.27) and (1.28) in Reference 1.

When analyzing composite structures, much of the intuition acquired from analysis of isotropic structures cannot be used. For instance, in the case of an isotropic 
structure possessing axisymmetric geometry under axisymmetric loads, no circumferential displacements will occur. Rather, in such a case, the structure can only expand/contract along the radial and axial directions. Hence, such a situation can be modeled by considering only a small sector (pie cut) and applying boundary conditions on the circumferential edges specifying that no circumferential displacements occur. However, in the case of a laminated composite structure possessing axisymmetric geometry under axisymmetric loads, circumferential displacements may or may not occur depending on the laminate stacking sequence. Thus, when a small sector is modeled for a composite structure, it is not correct to simply apply a condition of no circumferential displacements along the sector circumferential edges. Rather, true cyclic periodic boundary conditions must be used such that the sector modeled deforms in a way that the periodicity is preserved in the response. That is, the response is still axisymmetric in that it is independent of the angular position when expressed using an appropriate cylindrical coordinate system, even though it may involve circumferential displacements. For example, if a $10^{\circ}$ sector is modeled, the deformations must be such that taking 36 of these deformed $10^{\circ}$ sectors, the ends would still fit together to form a complete $360^{\circ}$ ring with no gaps.

In order to demonstrate these concepts clearly, various isotropic and composite rings under internal pressure will be examined. Two different ring geometries will be considered. The first ring geometry to be presented corresponds to a smaller radius, larger thickness, and a longer axial dimension than that for the second ring geometry which will be examined. Each ring is analyzed for various layer properties and stacking sequences. One goal of this ASC AD project has been to understand the differences between modeling thin structures using continuum and shell approaches in terms of the types of behavior that can be captured and in terms of computational cost. Hence, the rings will be analyzed using both continuum and shell element approaches. The linear elastic orthotropic shell and continuum models in ADAGIO/PRESTO have been verified with numerous orthotropic regression tests. Nevertheless, each modeling approach has its advantages and disadvantages/limitations. These differences will be explored presently. 


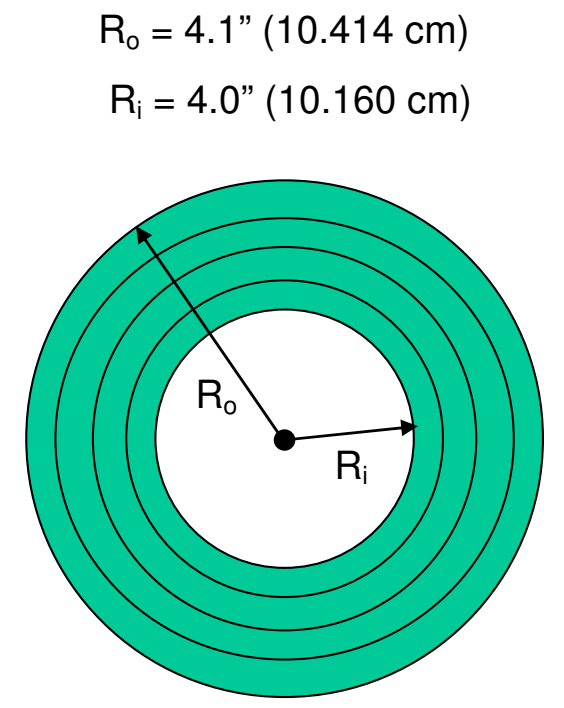

Figure 3.1: Ring A geometry.

\subsection{Ring A Problem Definition}

The "Ring A" geometry is as shown in Figure 3.1. Four layers each having a thickness of $0.025 "(0.0635 \mathrm{~cm})$ are used with an inner ring radius of 4.0" $(10.16 \mathrm{~cm})$. The ring has an axial length of $0.25 "(0.0635 \mathrm{~cm})$. Each lamina has the following orthotropic properties:

$$
\begin{aligned}
E_{11} & =64.03 \mathrm{GPa} \\
E_{22} & =55.50 \mathrm{GPa} \\
E_{33} & =9.78 \mathrm{GPa} \\
\nu_{12} & =0.081 \\
\nu_{23} & =0.303 \\
\nu_{31} & =0.0462805 \\
G_{12} & =G_{23}=G_{31}=4.45 \mathrm{GPa}
\end{aligned}
$$

Three composite laminate stacking sequences are considered: a symmetric cross-ply stacking sequence of [0/90/90/0], a uniform stacking sequence of [30/30/30/30], and a non-symmetric stacking sequence of $[10 / 50 / 60 / 30]$. Here the first layer in each stacking sequence corresponds to the outermost ring. A fiber angle of $0^{\circ}$ corresponds to the circumferential direction, while a fiber angle of $90^{\circ}$ corresponds to the axial direction. 
Other fiber angles are established by the appropriate rotation in the plane defined by the $0^{\circ}$ and $90^{\circ}$ fiber directions.

In order to demonstrate the validity of the cyclic periodic boundary conditions in ADAGIO, only a $10^{\circ}$ sector of each ring is modeled. The cyclic periodic boundary conditions applied on each circumferential edge allow for circumferential displacements. However, as previously noted, the circumferential displacements of the two circumferential edges must be compatible such that 36 of these $10^{\circ}$ sectors could be fit together to create a complete ring without any gaps in the deformed configuration. The composite rings for this ring geometry are modeled only using a continuum approach with solid brick elements.

\subsection{Ring A Results}

Each ring is subjected to an internal pressure of 100 psi. For the [0/90/90/0] cross-ply, the deformed shape is as shown in Figure 3.2. Such a symmetric cross-ply stacking sequence does not lead to any coupling between shear and normal behaviors or membrane and bending behaviors. Furthermore for this stacking sequence, the cross-ply stacking sequence is aligned with the cylindrical geometry such that the fibers are aligned in the circumferential and axial directions only. Hence, the deformations involve only radial and axial displacements. In such a case as this, explicitly constraining the circumferential displacements to be zero would not affect the solution.

The deformed mesh for the [30/30/30/30] composite ring is shown in Figures 3.3 and 3.4. Here, although there is no coupling between membrane and bending behaviors because the lay-up is symmetric about the middle surface, the shear and normal behaviors are coupled when viewed using the cylindrical coordinate system aligned naturally with the cylindrical ring geometry. That is, the membrane and bending normal stresses are coupled to the membrane and bending shear strains, respectively. Likewise, the membrane and bending shear stresses are coupled to the membrane and bending normal strains. It is precisely these couplings which lead to the shear behavior which is evident in the deformed geometry. Here is a case where if no circumferential displacements were applied to the circumferential edges of the $10^{\circ}$ wedge, the solution that would result would not correspond to the solution determined by modeling the entire ring. That is, applying no circumferential displacements to the $10^{\circ}$ wedge would 

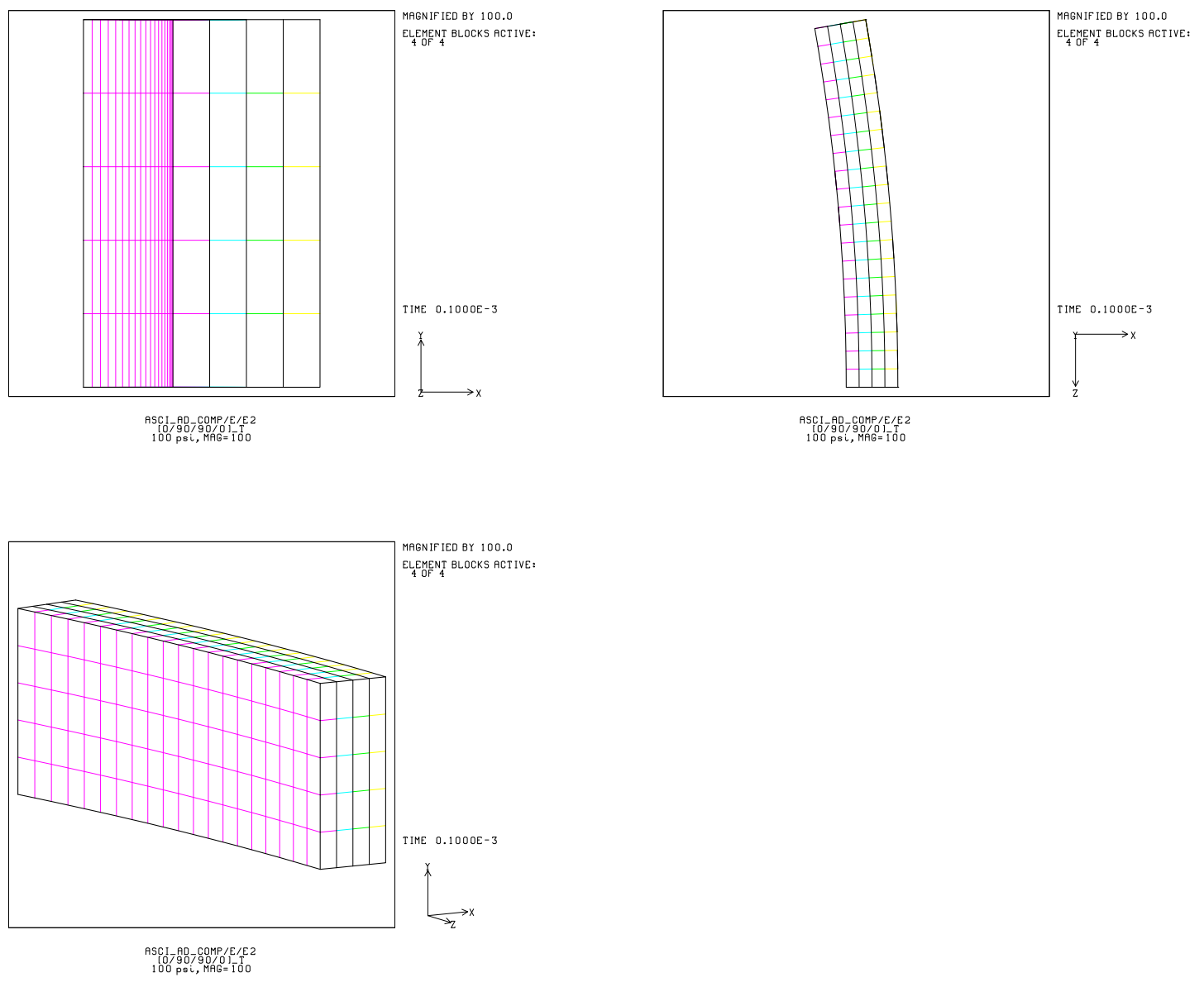

Figure 3.2: Deformed [0/90/90/0] composite Ring A (100X mag. of displacements). 

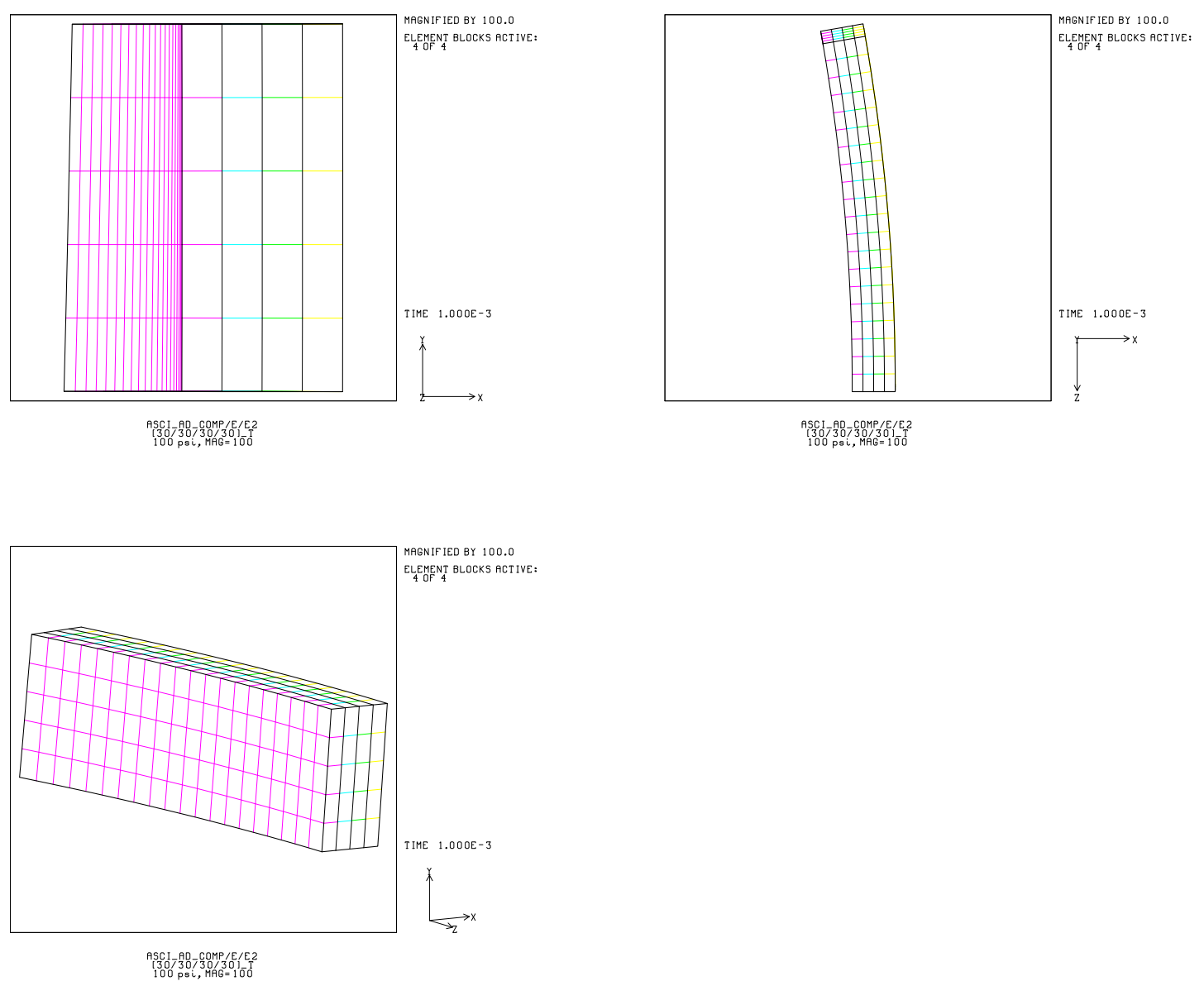

Figure 3.3: Deformed [30/30/30/30] composite Ring A (100X mag. of displacements).

overconstrain the solution.

Finally, the deformed mesh for the $[10 / 50 / 60 / 30]$ stacking sequence is shown in Figures 3.5 and 3.6. This stacking sequence involves coupling both between normal and shear behaviors and membrane and bending behaviors. The coupling between membrane and bending behaviors arises from the non-symmetric lay-up and links the membrane stresses to the bending strains as well as connecting the bending stresses to the membrane strains. Clearly, the deformed shape in this case is the most complex owing to its stacking sequence which has all possible couplings present. 

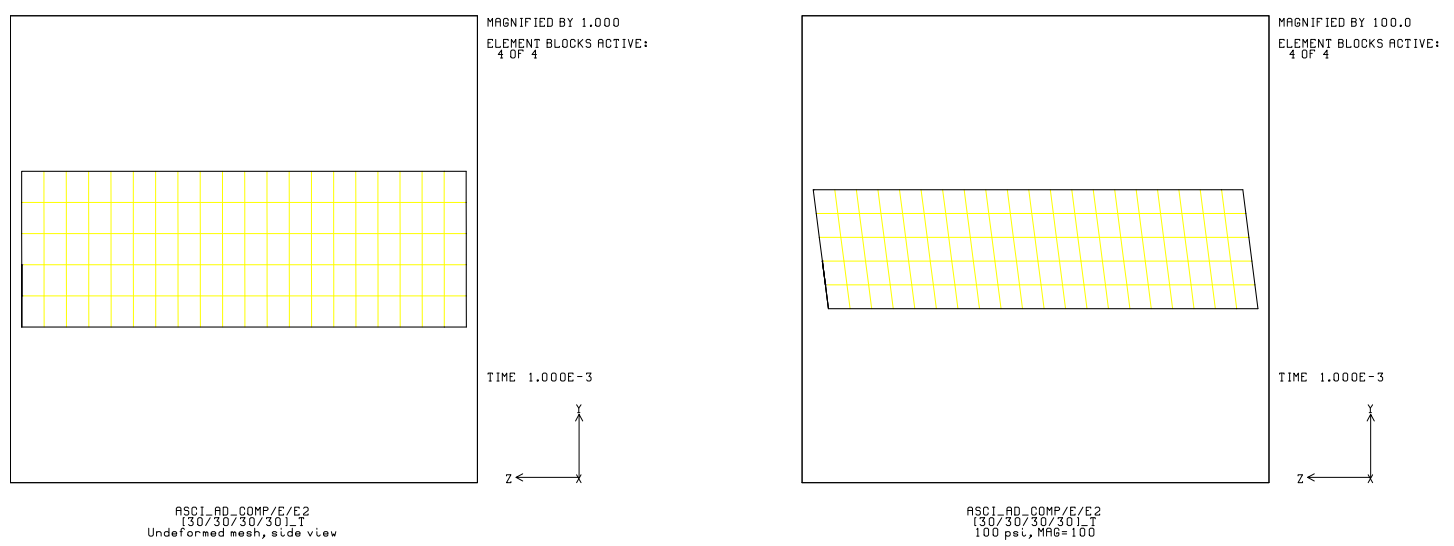

Figure 3.4: Undeformed and deformed side views of [30/30/30/30] composite Ring A (100X mag. of displacements).

\subsection{Ring B Problem Definition}

Like the Ring A problems just presented, the "Ring B" problems involve a 4-layer composite ring under internal pressure. However, Ring B has a larger internal radius of 7.908" and a smaller total thickness of 0.01". Stacking sequences of [0/90/90/0], [30/45/45/30], [30/-30/30/-30], and [30/60/50/10] are analyzed. In each case, the stacking sequence is given with the first angle corresponding to the outer layer with the remaining angles for the remaining layers working inward. The base material properties for each layer when viewed from a local coordinate system aligned with the 

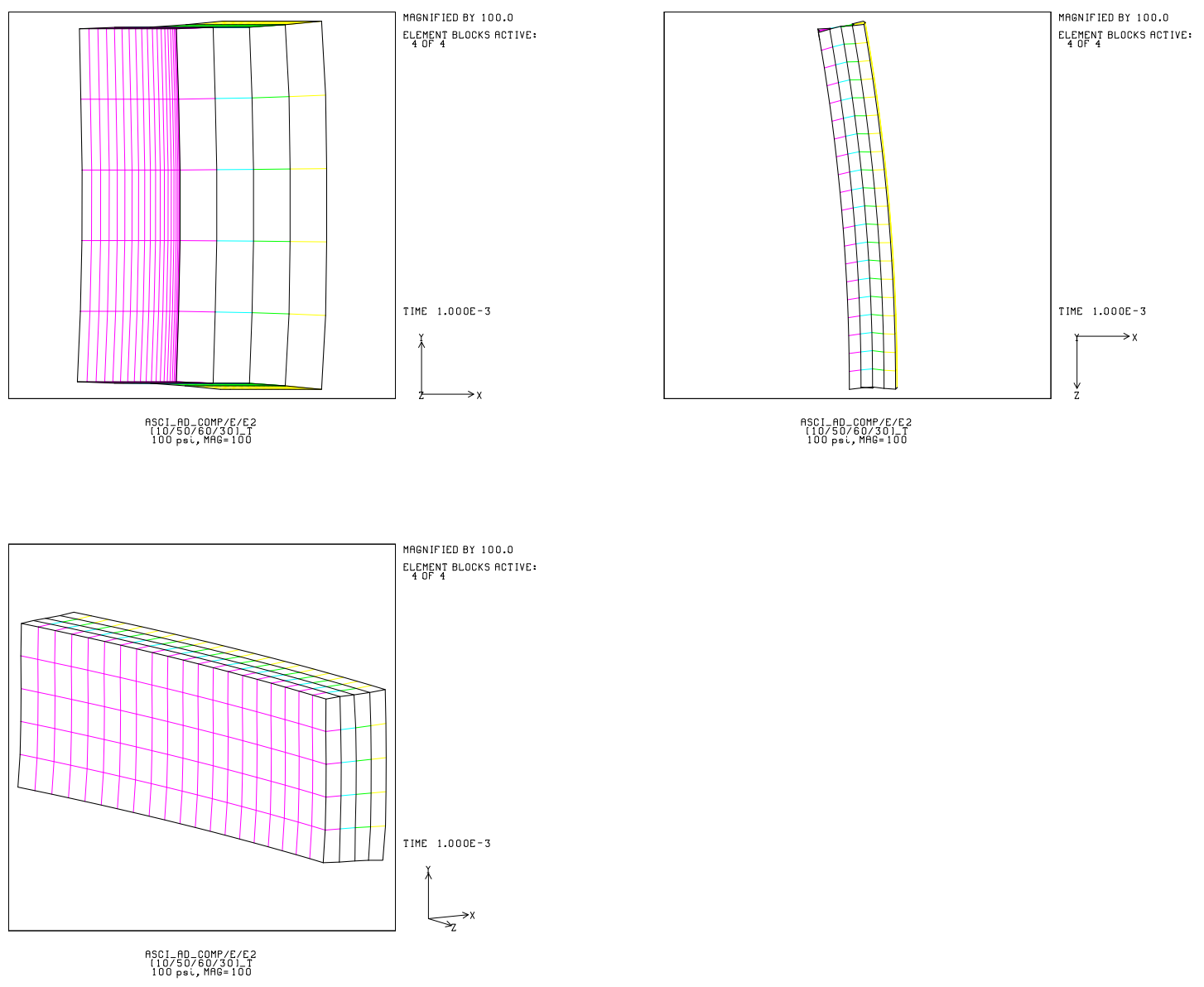

Figure 3.5: Deformed [10/50/60/30] composite Ring A (100X mag. of displacements).

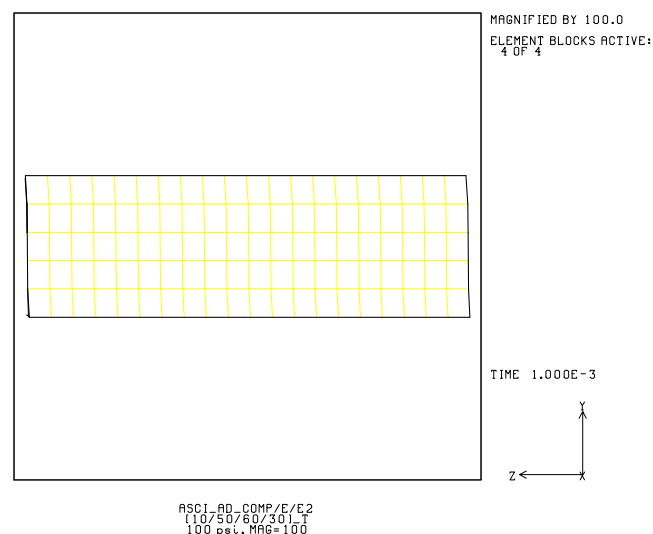

Figure 3.6: Deformed side view of $[10 / 50 / 60 / 30]$ composite Ring A (100X mag. of displacements). 
principal material directions are

$$
\begin{aligned}
E_{11} & =9.28691 \mathrm{Msi} \\
E_{22} & =8.04972 \mathrm{Msi} \\
E_{33} & =1.41849 \mathrm{Msi} \\
\nu_{12} & =0.081 \\
\nu_{23} & =0.303 \\
\nu_{31} & =0.0462805 \\
G_{12} & =0.645428 \mathrm{Msi} \\
G_{23} & =0.645428 \mathrm{Msi} \\
G_{31} & =0.645428 \mathrm{Msi} \\
\rho & =0.000140426 \frac{\mathrm{lb} \cdot \mathrm{s}^{2}}{\mathrm{in}^{4}}
\end{aligned}
$$

In addition to studying actual composite rings, an isotropic ring with the following material properties is also studied:

$$
\begin{aligned}
E & =9.28691 \mathrm{Msi} \\
\nu & =0.303 \\
\rho & =0.000140426 \frac{\mathrm{lb} \cdot \mathrm{s}^{2}}{\mathrm{in}^{4}}
\end{aligned}
$$

For each stacking sequence including the isotropic ring, the ring has a height of 0.1 ". In a further demonstration example, a [30/45/45/30] ring with a height of 0.01 ” is presented as a special case where the shell theory kinematic assumptions do not lead to high accuracy for some of the stress results. In all cases, the applied pressure magnitude is $1 \mathrm{psi}$ and the ring is free on all other edges. The ADAGIO input decks for the continuum and shell models corresponding to the [30/60/50/10] stacking sequence case are given in Appendices B and C, respectively.

In the case of an isotropic ring, simple results for the circumferential stress and expansion can be determined. Straightforward equilibrium calculations lead to the following equation for the hoop stress:

$$
\sigma_{\theta \theta}=\frac{p R}{t}
$$

where $\theta$ is the circumferential coordinate of the natural $r-\theta-z$ cylindrical coordinate 
system, $p$ is the applied pressure, $R$ is the nominal ring radius, and $t$ is the ring thickness. Because the only nonzero stress is the circumferential stress, the corresponding circumferential strain can then be determined as

$$
\epsilon_{\theta \theta}=\frac{\sigma_{\theta \theta}}{E}
$$

Finally, the radial deflection required to produce this circumferential strain is

$$
v_{r}=R_{\text {new }}-R_{\text {old }} \approx \epsilon_{\theta \theta} R_{\text {old }}
$$

For the chosen geometry and isotropic material properties, the following results are obtained:

$$
\begin{aligned}
\sigma_{\theta \theta} & =790.8 \mathrm{psi} \\
\epsilon_{\theta \theta} & =0.0000851521 \\
v_{r} & =0.000673 \mathrm{in} .
\end{aligned}
$$

\subsection{Ring B Results}

In the following pages, deformation and stress results are presented for all of the chosen rings. Also shown are the deformed geometries for all cases, and plots of strain, stress, or force and force-couple resultants for a few select cases. For all of the rings that are $0.1 "$ tall, a $0.5^{\circ}$ sector is modeled with periodic boundary conditions. For the additional $[30 / 45 / 45 / 30]$ ring which is $0.01 "$ tall, a $0.0625^{\circ}$ sector is modeled, again with periodic boundary conditions. For the plots of the deformed meshes, only the first column of elements is shown for convenience. Note that which entries are present in the laminate stiffness matrices that describe the laminate material response depend on the specific stacking sequence. A full range of stacking sequences have been chosen

all the way from an isotropic case to a random lay-up of [30/60/50/10] which has nonzero values for all entries in $[A],[B]$, and $[D]$.

Results for the various rings are presented in numerous tables and figures in this section. However, only an overview of the many results will be discussed subsequently.

For the isotropic case, the continuum and shell element results match very well. Moreover, the results from each for the radial displacement and circumferential stress match the mechanics of materials solution given by Eqs. (3.21)-(3.23). Similarly, excellent agreement between the two approaches results for the $[0 / 90 / 90 / 0]$ symmetric 
cross-ply stacking sequence. Note that the $[0 / 90 / 90 / 0]$ stacking sequence results in the same set of nonzero entries in $[A],\left[A_{t s}\right],[B]$, and $[D]$ as the isotropic case, but with, of course, different values. For both of these simple cases, the shell results required both fewer CG iterations and CPU time in ADAGIO.

For the $[30 / 45 / 45 / 30]$ symmetric stacking sequence, the laminate has coupling between normal and shear membrane stresses and strains (nonzero $A_{16}$ and $A_{26}$ ), in addition to coupling between normal and shear bending stresses and strains (nonzero $D_{16}$ and $\left.D_{26}\right)$. For this case, the agreement between the two approaches is reasonable for all results for the 0.1 " high ring. Note in Figure 3.11 which shows the deformed continuum model for this ring height, edge effects are quite apparent. However, the corresponding deformed shell model shown in Figure 3.12 does not exhibit such displacement edge effects. Such edge effects should become even more dominant for a smaller height ring. This is, in fact, what is observed for the [30/45/45/30] ring which is only 0.01 " in height. For this smaller ring height, reasonable agreement between the continuum and shell approaches result for the radial displacement and circumferential stress, but both the axial stress and the circumferential shear stress results differ significantly. In this particular case, care must be taken to achieve accurate results as demonstrated by the distribution of stresses shown in Figures 3.14 and 3.15. The corresponding force and force-couple resultants for the shell model are shown in Figures 3.17-3.19. Obviously, since only a single element is used in the thickness direction under the assumptions of Mindlin shell theory, it is not possible for the shell model to capture the actual stress variation in the radial direction, or the curving of lines which are originally purely radial in direction.

The antisymmetric angle-ply stacking sequence of $[30 /-30 / 30 /-30]$ does not have coupling between normal and shear membrane stresses and strains or coupling between normal and shear bending stresses and strains. However, it does have coupling between the shear bending strains and the normal membrane stresses, the normal bending strains and the shear membrane stresses, the shear membrane strains and the normal bending stresses, and the normal membrane strains and the shear bending stresses (nonzero $B_{16}$ and $B_{26}$ ). For this particular case, the agreement between the two approaches for the radial deflection appears to be reasonable. On the other hand, most of the stress results are off. For instance, the circumferential stress results are 
very different in nature with the continuum model giving circumferential stresses which are similar in each layer, but the shell model giving values that differ between the inner and outer layers. Moreover, the shell model predicts nonzero axial stresses which sum to zero over the four layers, whereas the continuum model predicts all layers to have zero axial stress. Finally, although the circumferential shear stress results have the same sign for each layer in the two approaches, the shell model predicts a big variation in the value of this stress between the inner and outer layers, whereas the continuum model does not. The variation in layer stresses coming from the shell model is seen to arise from its prediction of a significant bending shear strain as shown in Figure 3.24. In this and most other cases, presumably the continuum model results are to be trusted more than the shell results, because the continuum model is based on less restrictive kinematic assumptions than the shell model. Note that for this particular stacking sequence, the shell and continuum models required comparable computational effort.

Finally, the somewhat contrived [30/60/50/10] lay-up has all terms present in $[A],[B]$, and $[D]$ leading to all possible couplings between membrane and bending stresses and strains. However, the off-diagonal terms in $\left[A_{t s}\right]$ are still zero, as the two transverse shear moduli are taken to be equal. For this particular case, reasonable agreement exists between the shell and continuum results for all quantities examined. Note in Figures 3.25 and 3.26 the relatively significant bending that results for this stacking sequence which has a full membrane/bending coupling matrix $[B]$. In this particular case, the shell model required significantly more computational effort than the continuum model. 
Table 3.1: Radial displacement at middle of inside surface for isotropic Ring B.

\begin{tabular}{ll} 
Case & $\begin{array}{l}V_{r} \\
\text { (in.) }\end{array}$ \\
\hline \hline Mech. of Mat. & 0.000673 \\
Cont. FEM & 0.000674161 \\
Shell FEM & 0.000673471 \\
\hline \hline
\end{tabular}

Table 3.2: Stress $\sigma_{X X}=\sigma_{r r}$ at ring center for isotropic Ring B.

\begin{tabular}{lllll} 
Case & $\begin{array}{l}\text { L1 } \\
(\mathrm{psi})\end{array}$ & $\begin{array}{l}\text { L2 } \\
(\mathrm{psi})\end{array}$ & $\begin{array}{l}\text { L3 } \\
(\mathrm{psi})\end{array}$ & $\begin{array}{l}\text { L4 } \\
(\mathrm{psi})\end{array}$ \\
\hline \hline Mech. of Mat. & 0.0 & 0.0 & 0.0 & 0.0 \\
Cont. FEM & $-1.285492 \mathrm{E}-01$ & $-3.789259 \mathrm{E}-01$ & $-6.307772 \mathrm{E}-01$ & $-8.768584 \mathrm{E}-01$ \\
Shell FEM & $-1.322894 \mathrm{E}-10$ & $-1.322894 \mathrm{E}-10$ & $-1.322894 \mathrm{E}-10$ & $-1.322894 \mathrm{E}-10$ \\
\hline \hline
\end{tabular}

Table 3.3: Stress $\sigma_{Y Y}=\sigma_{z z}$ at ring center for isotropic Ring B.

\begin{tabular}{lllll} 
Case & $\begin{array}{l}\text { L1 } \\
(\mathrm{psi})\end{array}$ & $\begin{array}{l}\text { L2 } \\
(\mathrm{psi})\end{array}$ & $\begin{array}{l}\text { L3 } \\
(\mathrm{psi})\end{array}$ & $\begin{array}{l}\text { L4 } \\
(\mathrm{psi})\end{array}$ \\
\hline \hline Mech. of Mat. & 0.0 & 0.0 & 0.0 & 0.0 \\
Cont. FEM & $9.381270 \mathrm{E}-03$ & $4.009083 \mathrm{E}-03$ & $-7.445849 \mathrm{E}-03$ & $-1.784666 \mathrm{E}-02$ \\
Shell FEM & $-2.792358 \mathrm{E}-02$ & $-1.004028 \mathrm{E}-02$ & $7.873535 \mathrm{E}-03$ & $2.575684 \mathrm{E}-02$ \\
\hline \hline
\end{tabular}

Table 3.4: Stress $\sigma_{Z Z}=\sigma_{\theta \theta}$ at ring center for isotropic Ring B.

\begin{tabular}{llllll} 
Case & $\begin{array}{l}\text { L1 } \\
(\mathrm{psi})\end{array}$ & $\begin{array}{l}\text { L2 } \\
(\mathrm{psi})\end{array}$ & $\begin{array}{l}\text { L3 } \\
\text { (psi) }\end{array}$ & $\begin{array}{l}\text { L4 } \\
(\mathrm{psi})\end{array}$ & $\begin{array}{l}\text { AVG } \\
\text { (psi) }\end{array}$ \\
\hline \hline Mech. of Mat. & 790.8000 & 790.8000 & 790.8000 & 790.8000 & 790.8000 \\
Cont. FEM & 790.5351 & 790.7678 & 790.9818 & 791.1935 & 790.8696 \\
Shell FEM & 790.7740 & 790.8325 & 790.8911 & 790.9496 & 790.8618 \\
\hline \hline
\end{tabular}


Table 3.5: Stress $\sigma_{X Y}=\sigma_{z r}$ at ring center for isotropic Ring B.

\begin{tabular}{lllll} 
Case & $\begin{array}{l}\text { L1 } \\
(\mathrm{psi})\end{array}$ & $\begin{array}{l}\text { L2 } \\
(\mathrm{psi})\end{array}$ & $\begin{array}{l}\text { L3 } \\
(\mathrm{psi})\end{array}$ & $\begin{array}{l}\text { L4 } \\
(\mathrm{psi})\end{array}$ \\
\hline \hline Mech. of Mat. & 0.0 & 0.0 & 0.0 & 0.0 \\
Cont. FEM & $8.828444 \mathrm{E}-07$ & $3.358880 \mathrm{E}-06$ & $2.407217 \mathrm{E}-06$ & $1.432775 \mathrm{E}-06$ \\
Shell FEM & $-4.016570 \mathrm{E}-05$ & $-4.016575 \mathrm{E}-05$ & $-4.016580 \mathrm{E}-05$ & $-4.016585 \mathrm{E}-05$ \\
\hline \hline
\end{tabular}

Table 3.6: Stress $\sigma_{Y Z}=-\sigma_{\theta z}$ at ring center for isotropic Ring B.

\begin{tabular}{lllll} 
Case & $\begin{array}{l}\text { L1 } \\
(\mathrm{psi})\end{array}$ & $\begin{array}{l}\text { L2 } \\
(\mathrm{psi})\end{array}$ & $\begin{array}{l}\text { L3 } \\
(\mathrm{psi})\end{array}$ & $\begin{array}{l}\mathrm{L} 4 \\
(\mathrm{psi})\end{array}$ \\
\hline \hline Mech. of Mat. & 0.0 & 0.0 & 0.0 & 0.0 \\
Cont. FEM & $-6.401723 \mathrm{E}-05$ & $-6.235721 \mathrm{E}-05$ & $-6.138064 \mathrm{E}-05$ & $-6.052429 \mathrm{E}-05$ \\
Shell FEM & $7.139375 \mathrm{E}-06$ & $2.592917 \mathrm{E}-06$ & $-1.953540 \mathrm{E}-06$ & $-6.499996 \mathrm{E}-06$ \\
\hline \hline
\end{tabular}

Table 3.7: Stress $\sigma_{Z X}=-\sigma_{r \theta}$ at ring center for isotropic Ring B.

\begin{tabular}{lllll} 
Case & $\begin{array}{l}\text { L1 } \\
(\mathrm{psi})\end{array}$ & $\begin{array}{l}\text { L2 } \\
(\mathrm{psi})\end{array}$ & $\begin{array}{l}\text { L3 } \\
(\mathrm{psi})\end{array}$ & $\begin{array}{l}\text { L4 } \\
(\mathrm{psi})\end{array}$ \\
\hline \hline Mech. of Mat. & 0.0 & 0.0 & 0.0 & 0.0 \\
Cont. FEM & $3.224001 \mathrm{E}-03$ & $-8.411866 \mathrm{E}-03$ & $-2.593525 \mathrm{E}-03$ & $1.459236 \mathrm{E}-02$ \\
Shell FEM & $3.944047 \mathrm{E}-03$ & $3.944046 \mathrm{E}-03$ & $3.944045 \mathrm{E}-03$ & $3.944044 \mathrm{E}-03$ \\
\hline \hline
\end{tabular}

Table 3.8: Computational cost for isotropic Ring B.

\begin{tabular}{lllllll} 
Case & Model & Elements & Nodes & DOF w/o BC & Iterations & $\begin{array}{c}\text { CPU } \\
\text { (sec) }\end{array}$ \\
\hline \hline Cont. FEM & 0.5 deg & 800 & 1155 & 3465 & 518 & 5.779 \\
Shell FEM & $0.5 \mathrm{deg}$ & 200 & 231 & 1386 & 230 & 2.290 \\
\hline \hline
\end{tabular}



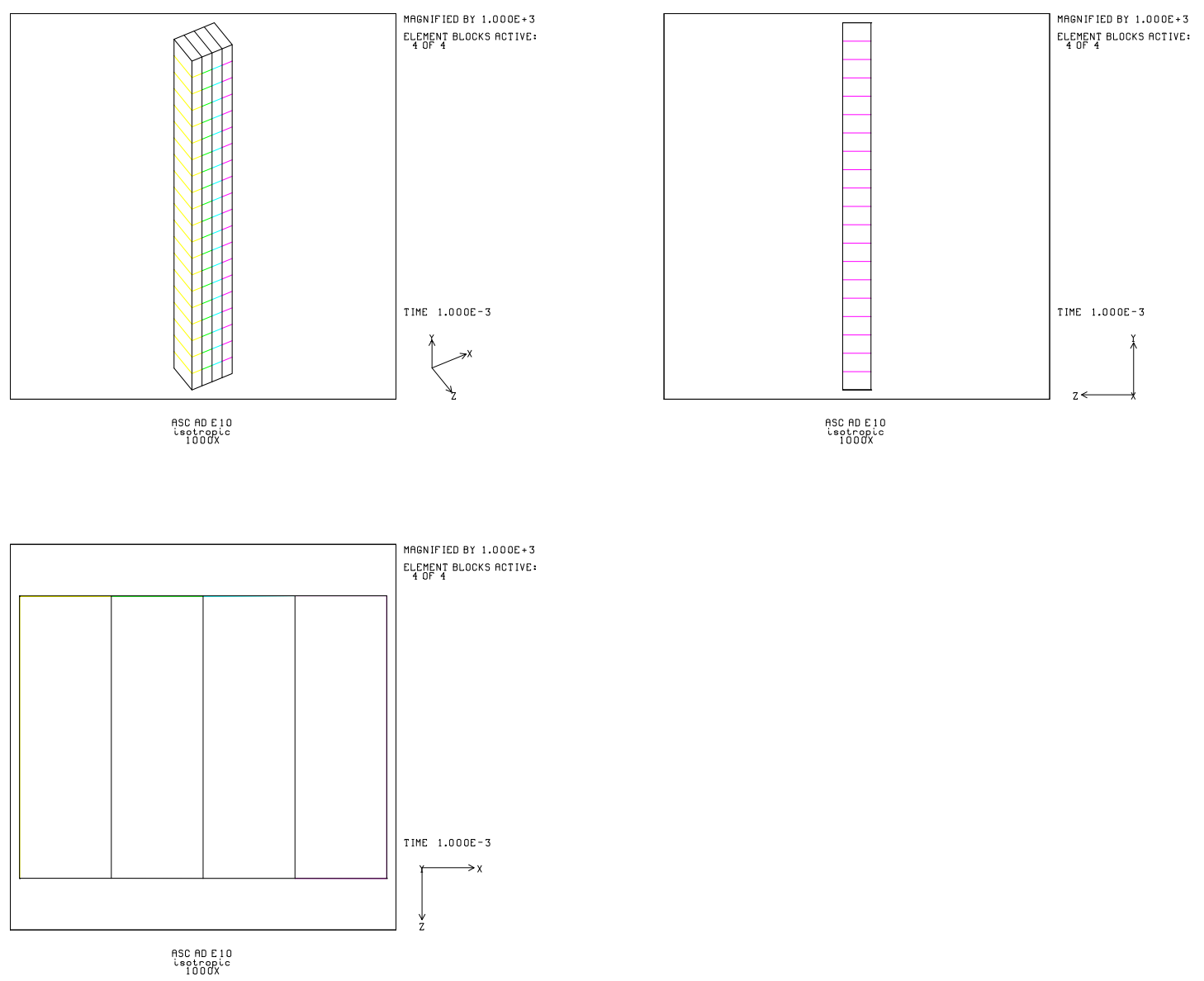

Figure 3.7: Deformed isotropic Ring B continuum model showing 1 element in circumferential direction. Deformations magnified by 1000 . 

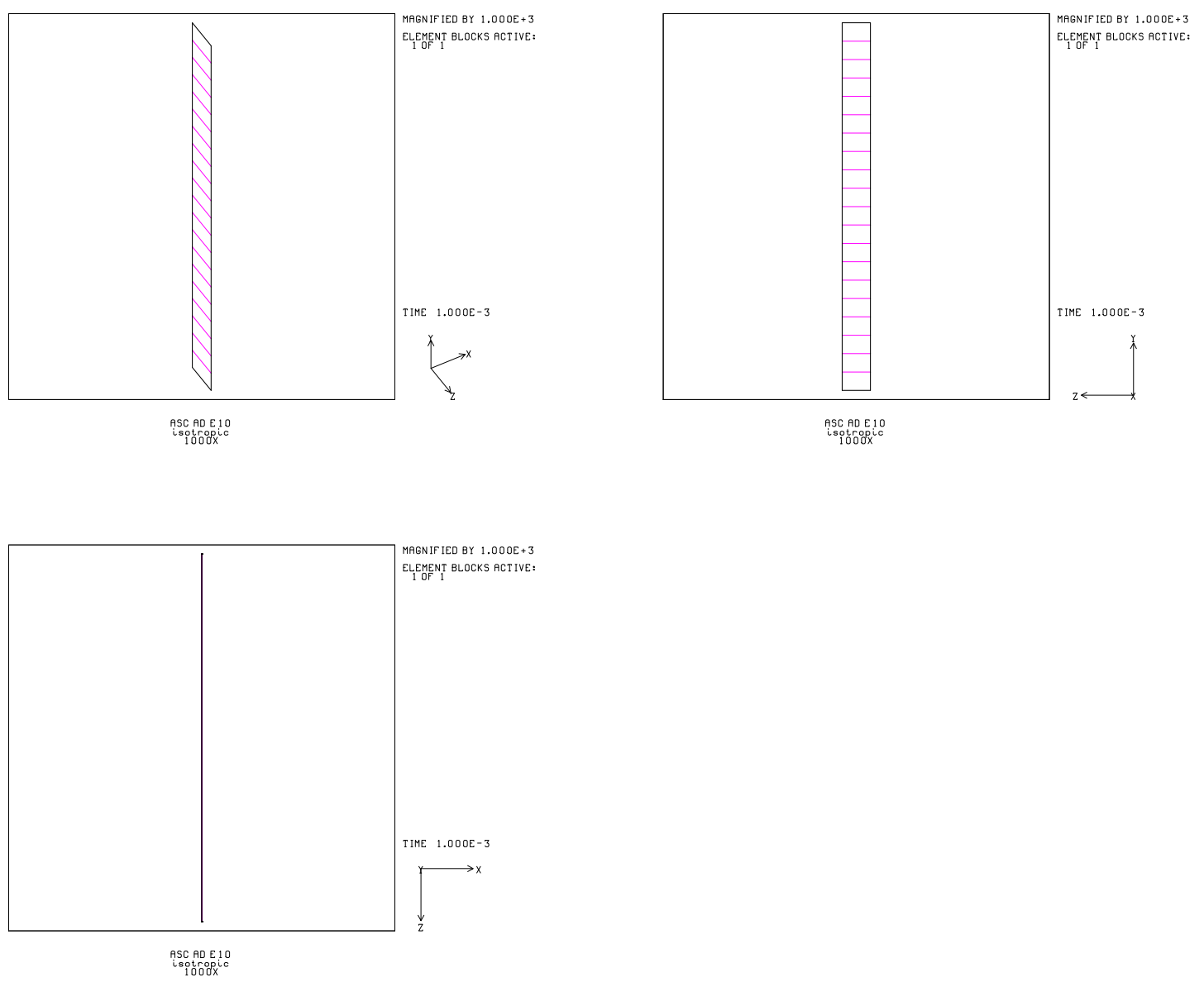

Figure 3.8: Deformed isotropic Ring B shell model showing 1 element in circumferential direction. Deformations magnified by 1000 . 
Table 3.9: Radial displacement at middle of inside surface for [0/90/90/0] Ring B.

\begin{tabular}{ll} 
Case & $\begin{array}{l}V_{r} \\
\text { (in.) }\end{array}$ \\
\hline \hline Cont. FEM & 0.00072226 \\
Shell FEM & 0.000721516 \\
\hline \hline
\end{tabular}

Table 3.10: Stress $\sigma_{X X}=\sigma_{r r}$ at ring center for [0/90/90/0] Ring B.

\begin{tabular}{lllll} 
Case & $\begin{array}{l}\text { L1 } \\
(\mathrm{psi})\end{array}$ & $\begin{array}{l}\text { L2 } \\
(\mathrm{psi})\end{array}$ & $\begin{array}{l}\text { L3 } \\
(\mathrm{psi})\end{array}$ & $\begin{array}{l}\text { L4 } \\
(\mathrm{psi})\end{array}$ \\
\hline \hline Cont. FEM & $-1.340192 \mathrm{E}-01$ & $-3.844722 \mathrm{E}-01$ & $-6.194120 \mathrm{E}-01$ & $-8.685544 \mathrm{E}-01$ \\
Shell FEM & $-1.033863 \mathrm{E}-10$ & $-1.034079 \mathrm{E}-10$ & $-1.034079 \mathrm{E}-10$ & $-1.033864 \mathrm{E}-10$ \\
\hline \hline
\end{tabular}

Table 3.11: Stress $\sigma_{Y Y}=\sigma_{z z}$ at ring center for [0/90/90/0] Ring B.

\begin{tabular}{lllll} 
Case & $\begin{array}{l}\text { L1 } \\
(\mathrm{psi})\end{array}$ & $\begin{array}{l}\text { L2 } \\
(\mathrm{psi})\end{array}$ & $\begin{array}{l}\text { L3 } \\
(\mathrm{psi})\end{array}$ & \multicolumn{1}{l}{$\begin{array}{l}\mathrm{L} 4 \\
(\mathrm{psi})\end{array}$} \\
\hline \hline Cont. FEM & $4.265626 \mathrm{E}+00$ & $-4.262483 \mathrm{E}+00$ & $-4.255891 \mathrm{E}+00$ & $4.250383 \mathrm{E}+00$ \\
Shell FEM & $4.275406 \mathrm{E}+00$ & $-4.267586 \mathrm{E}+00$ & $-4.271603 \mathrm{E}+00$ & $4.263306 \mathrm{E}+00$ \\
\hline \hline
\end{tabular}

Table 3.12: Stress $\sigma_{Z Z}=\sigma_{\theta \theta}$ at ring center for [0/90/90/0] Ring B.

\begin{tabular}{llllll} 
Case & $\begin{array}{l}\text { L1 } \\
(\mathrm{psi})\end{array}$ & $\begin{array}{l}\text { L2 } \\
(\mathrm{psi})\end{array}$ & $\begin{array}{l}\text { L3 } \\
(\mathrm{psi})\end{array}$ & $\begin{array}{l}\text { L4 } \\
(\mathrm{psi})\end{array}$ & $\begin{array}{l}\text { AVG } \\
(\mathrm{psi})\end{array}$ \\
\hline \hline Cont. FEM & 847.2699 & 734.0089 & 734.2285 & 848.0016 & 790.8772 \\
Shell FEM & 847.7199 & 734.1360 & 734.0841 & 847.5402 & 790.8701 \\
\hline \hline
\end{tabular}

Table 3.13: Stress $\sigma_{X Y}=\sigma_{z r}$ at ring center for [0/90/90/0] Ring B.

\begin{tabular}{lllll} 
Case & $\begin{array}{l}\text { L1 } \\
(\mathrm{psi})\end{array}$ & $\begin{array}{l}\text { L2 } \\
(\mathrm{psi})\end{array}$ & $\begin{array}{l}\text { L3 } \\
(\mathrm{psi})\end{array}$ & $\begin{array}{l}\text { L4 } \\
(\mathrm{psi})\end{array}$ \\
\hline \hline Cont. FEM & $-1.181300 \mathrm{E}-05$ & $-1.513988 \mathrm{E}-05$ & $8.299375 \mathrm{E}-06$ & $8.985447 \mathrm{E}-06$ \\
Shell FEM & $-1.835007 \mathrm{E}-05$ & $-1.834918 \mathrm{E}-05$ & $-1.834918 \mathrm{E}-05$ & $-1.835007 \mathrm{E}-05$ \\
\hline \hline
\end{tabular}


Table 3.14: Stress $\sigma_{Y Z}=-\sigma_{\theta z}$ at ring center for $[0 / 90 / 90 / 0]$ Ring B.

\begin{tabular}{lllll} 
Case & $\begin{array}{l}\text { L1 } \\
(\mathrm{psi})\end{array}$ & $\begin{array}{l}\text { L2 } \\
(\mathrm{psi})\end{array}$ & $\begin{array}{l}\text { L3 } \\
(\mathrm{psi})\end{array}$ & $\begin{array}{l}\text { L4 } \\
(\mathrm{psi})\end{array}$ \\
\hline \hline Cont. FEM & $-2.408314 \mathrm{E}-05$ & $7.427691 \mathrm{E}-06$ & $6.961428 \mathrm{E}-06$ & $-2.420312 \mathrm{E}-05$ \\
Shell FEM & $4.575826 \mathrm{E}-06$ & $1.429134 \mathrm{E}-06$ & $-1.717616 \mathrm{E}-06$ & $-4.864422 \mathrm{E}-06$ \\
\hline \hline
\end{tabular}

Table 3.15: Stress $\sigma_{Z X}=-\sigma_{r \theta}$ at ring center for [0/90/90/0] Ring B.

\begin{tabular}{lllll} 
Case & $\begin{array}{l}\text { L1 } \\
(\mathrm{psi})\end{array}$ & $\begin{array}{l}\text { L2 } \\
(\mathrm{psi})\end{array}$ & $\begin{array}{l}\text { L3 } \\
(\mathrm{psi})\end{array}$ & $\begin{array}{l}\text { L4 } \\
(\mathrm{psi})\end{array}$ \\
\hline \hline Cont. FEM & $7.271318 \mathrm{E}-04$ & $-5.612549 \mathrm{E}-03$ & $-2.052617 \mathrm{E}-03$ & $1.376524 \mathrm{E}-02$ \\
Shell FEM & $3.746277 \mathrm{E}-03$ & $3.747842 \mathrm{E}-03$ & $3.747843 \mathrm{E}-03$ & $3.746279 \mathrm{E}-03$ \\
\hline \hline
\end{tabular}

Table 3.16: Computational cost for [0/90/90/0] Ring B.

\begin{tabular}{lllllll} 
Case & Model & Elements & Nodes & DOF w/o BC & Iterations & $\begin{array}{c}\text { CPU } \\
\text { (sec) }\end{array}$ \\
\hline \hline Cont. FEM & $0.5 \mathrm{deg}$ & 800 & 1155 & 3465 & 643 & 8.859 \\
Shell FEM & $0.5 \mathrm{deg}$ & 200 & 231 & 1386 & 369 & 3.180 \\
\hline \hline
\end{tabular}



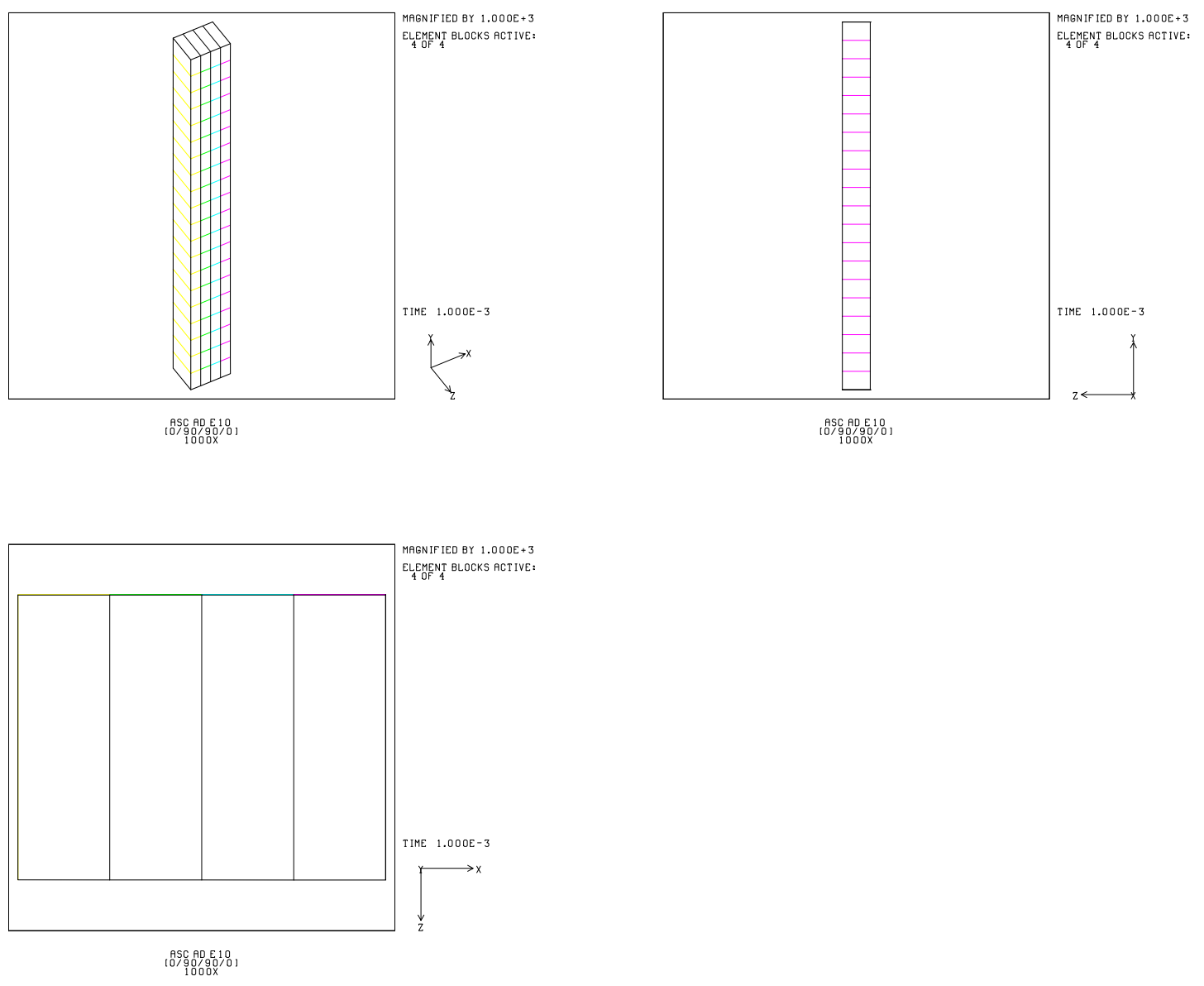

Figure 3.9: Deformed [0/90/90/0] Ring B continuum model showing 1 element in circumferential direction. Deformations magnified by 1000. 

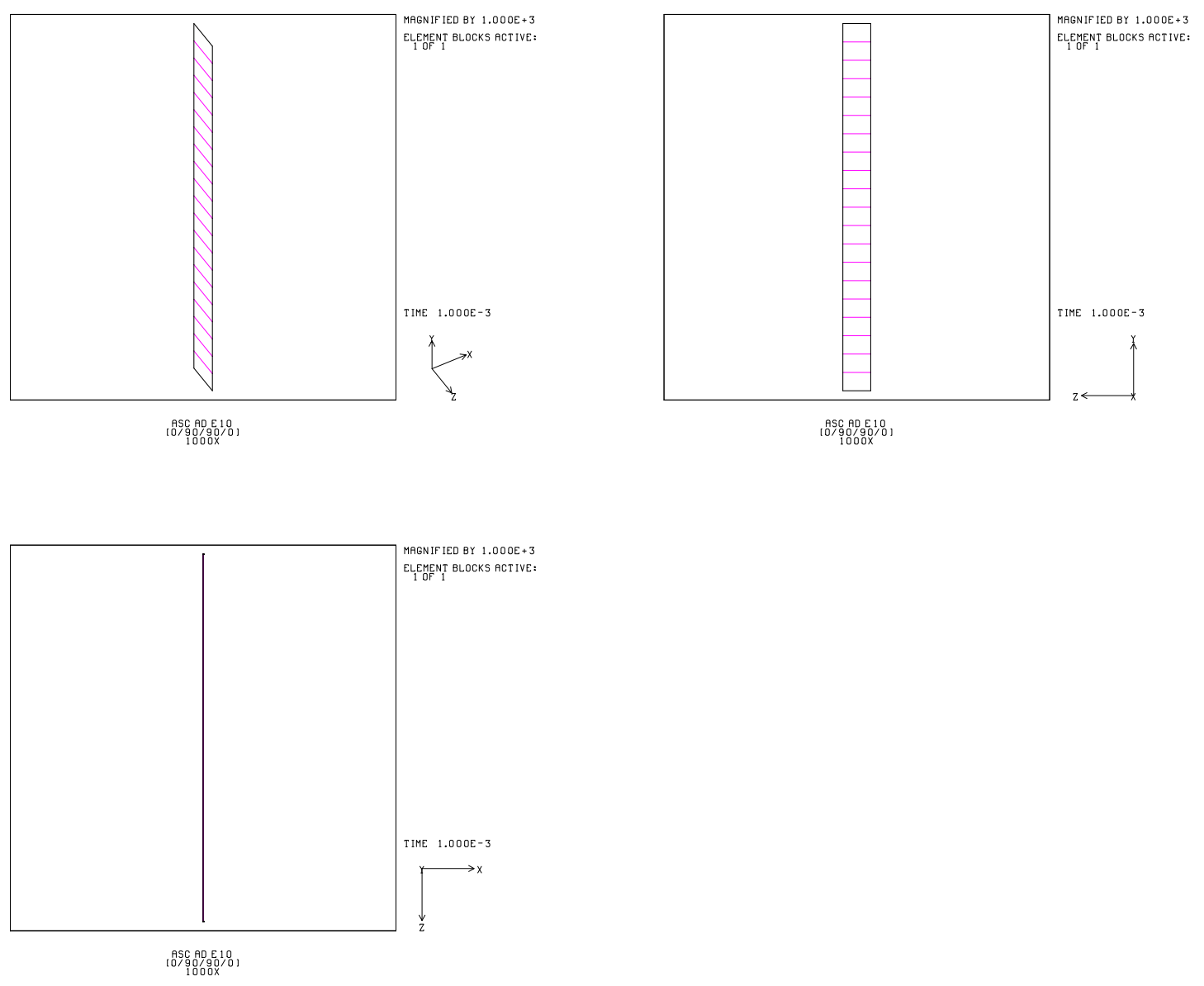

Figure 3.10: Deformed [0/90/90/0] Ring B shell model showing 1 element in circumferential direction. Deformations magnified by 1000. 
Table 3.17: Radial displacement at middle of inside surface for 0.1" high [30/45/30/30] Ring B.

\begin{tabular}{ll} 
Case & $\begin{array}{l}V_{r} \\
\text { (in.) }\end{array}$ \\
\hline \hline Cont. FEM & 0.00204457 \\
Shell FEM & 0.00202239 \\
\hline \hline
\end{tabular}

Table 3.18: Stress $\sigma_{X X}=\sigma_{r r}$ at ring center for 0.1" high [30/45/30/30] Ring B.

\begin{tabular}{lllll} 
Case & $\begin{array}{l}\text { L1 } \\
(\mathrm{psi})\end{array}$ & $\begin{array}{l}\text { L2 } \\
(\mathrm{psi})\end{array}$ & $\begin{array}{l}\text { L3 } \\
(\mathrm{psi})\end{array}$ & \multicolumn{1}{l}{$\begin{array}{l}\text { L4 } \\
(\mathrm{psi})\end{array}$} \\
\hline \hline Cont. FEM & $-1.518291 \mathrm{E}-01$ & $-4.006721 \mathrm{E}-01$ & $-6.055231 \mathrm{E}-01$ & $-8.550707 \mathrm{E}-01$ \\
Shell FEM & $-4.583551 \mathrm{E}-10$ & $-4.592669 \mathrm{E}-10$ & $-4.592670 \mathrm{E}-10$ & $-4.583556 \mathrm{E}-10$ \\
\hline \hline
\end{tabular}

Table 3.19: Stress $\sigma_{Y Y}=\sigma_{z z}$ at ring center for 0.1" high [30/45/30/30] Ring B.

\begin{tabular}{lllll} 
Case & $\begin{array}{l}\text { L1 } \\
(\mathrm{psi})\end{array}$ & $\begin{array}{l}\text { L2 } \\
(\mathrm{psi})\end{array}$ & $\begin{array}{l}\text { L3 } \\
(\mathrm{psi})\end{array}$ & $\begin{array}{l}\text { L4 } \\
(\mathrm{psi})\end{array}$ \\
\hline \hline Cont. FEM & -85.04935 & 84.39281 & 84.80602 & -84.15880 \\
Shell FEM & -83.72532 & 83.80194 & 83.76256 & -83.83939 \\
\hline \hline
\end{tabular}

Table 3.20: Stress $\sigma_{Z Z}=\sigma_{\theta \theta}$ at ring center for 0.1" high [30/45/30/30] Ring B.

\begin{tabular}{llllll} 
Case & $\begin{array}{l}\text { L1 } \\
(\mathrm{psi})\end{array}$ & $\begin{array}{l}\text { L2 } \\
(\mathrm{psi})\end{array}$ & $\begin{array}{l}\text { L3 } \\
(\mathrm{psi})\end{array}$ & $\begin{array}{l}\text { L4 } \\
(\mathrm{psi})\end{array}$ & $\begin{array}{l}\text { AVG } \\
(\mathrm{psi})\end{array}$ \\
\hline \hline Cont. FEM & 953.9507 & 642.9663 & 643.4601 & 955.7718 & 799.0372 \\
Shell FEM & 945.3959 & 636.7208 & 636.6677 & 945.2206 & 791.0013 \\
\hline \hline
\end{tabular}

Table 3.21: Stress $\sigma_{X Y}=\sigma_{z r}$ at ring center for 0.1" high [30/45/30/30] Ring B.

\begin{tabular}{lllll} 
Case & $\begin{array}{l}\text { L1 } \\
(\mathrm{psi})\end{array}$ & $\begin{array}{l}\text { L2 } \\
(\mathrm{psi})\end{array}$ & $\begin{array}{l}\text { L3 } \\
(\mathrm{psi})\end{array}$ & $\begin{array}{l}\text { L4 } \\
(\mathrm{psi})\end{array}$ \\
\hline \hline Cont. FEM & $-4.364775 \mathrm{E}-03$ & $1.234140 \mathrm{E}-02$ & $1.218694 \mathrm{E}-02$ & $-8.669811 \mathrm{E}-03$ \\
Shell FEM & $-9.679240 \mathrm{E}-05$ & $-1.342813 \mathrm{E}-04$ & $-1.342804 \mathrm{E}-04$ & $-9.679284 \mathrm{E}-05$ \\
\hline \hline
\end{tabular}


Table 3.22: Stress $\sigma_{Y Z}=-\sigma_{\theta z}$ at ring center for 0.1" high [30/45/30/30] Ring B.

\begin{tabular}{lllll} 
Case & $\begin{array}{l}\text { L1 } \\
(\mathrm{psi})\end{array}$ & $\begin{array}{l}\text { L2 } \\
(\mathrm{psi})\end{array}$ & $\begin{array}{l}\text { L3 } \\
(\mathrm{psi})\end{array}$ & $\begin{array}{l}\text { L4 } \\
(\mathrm{psi})\end{array}$ \\
\hline \hline Cont. FEM & -355.1625 & 355.5782 & 355.4099 & -355.8285 \\
Shell FEM & -351.9032 & 351.9069 & 351.8897 & -351.8960 \\
\hline \hline
\end{tabular}

Table 3.23: Stress $\sigma_{Z X}=-\sigma_{r \theta}$ at ring center for 0.1" high [30/45/30/30] Ring B.

\begin{tabular}{lllll} 
Case & $\begin{array}{l}\text { L1 } \\
(\mathrm{psi})\end{array}$ & $\begin{array}{l}\text { L2 } \\
(\mathrm{psi})\end{array}$ & $\begin{array}{l}\text { L3 } \\
(\mathrm{psi})\end{array}$ & $\begin{array}{l}\text { L4 } \\
(\mathrm{psi})\end{array}$ \\
\hline \hline Cont. FEM & $4.623912 \mathrm{E}-03$ & $-1.053747 \mathrm{E}-02$ & $-5.237671 \mathrm{E}-03$ & $1.940667 \mathrm{E}-02$ \\
Shell FEM & $4.268043 \mathrm{E}-03$ & $4.284822 \mathrm{E}-03$ & $4.284825 \mathrm{E}-03$ & $4.268053 \mathrm{E}-03$ \\
\hline \hline
\end{tabular}

Table 3.24: Computational cost for 0.1" high [30/45/30/30] Ring B.

\begin{tabular}{lllllll} 
Case & Model & Elements & Nodes & DOF w/o BC & Iterations & $\begin{array}{l}\text { CPU } \\
(\mathrm{sec})\end{array}$ \\
\hline \hline Cont. FEM & $0.5 \mathrm{deg}$ & 800 & 1155 & 3465 & 1258 & 16.309 \\
Shell FEM & $0.5 \mathrm{deg}$ & 200 & 231 & 1386 & 1983 & 13.509 \\
\hline \hline
\end{tabular}



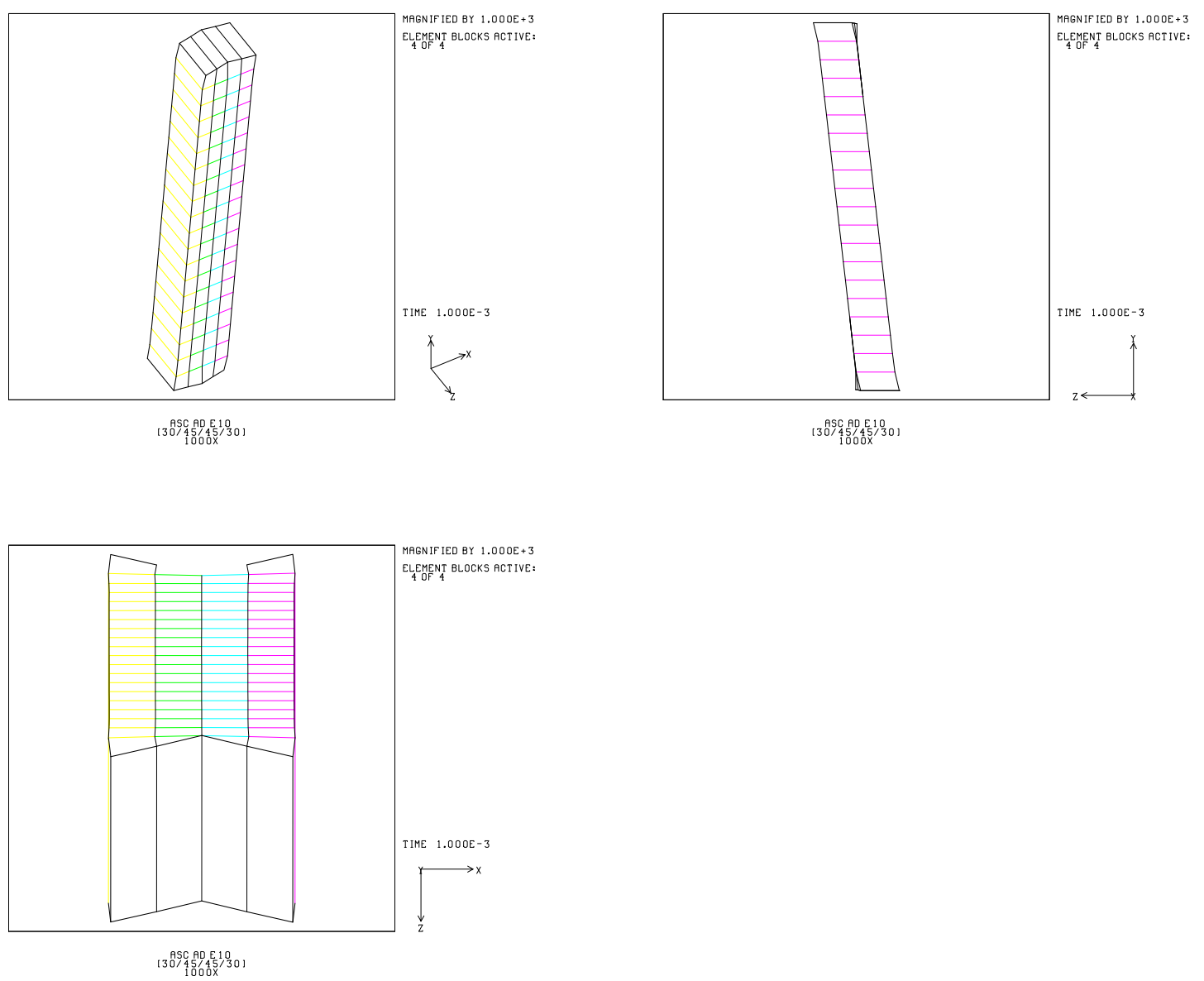

Figure 3.11: Deformed 0.1" high [30/45/45/30] Ring B continuum model showing 1 element in circumferential direction. Deformations magnified by 1000 . 

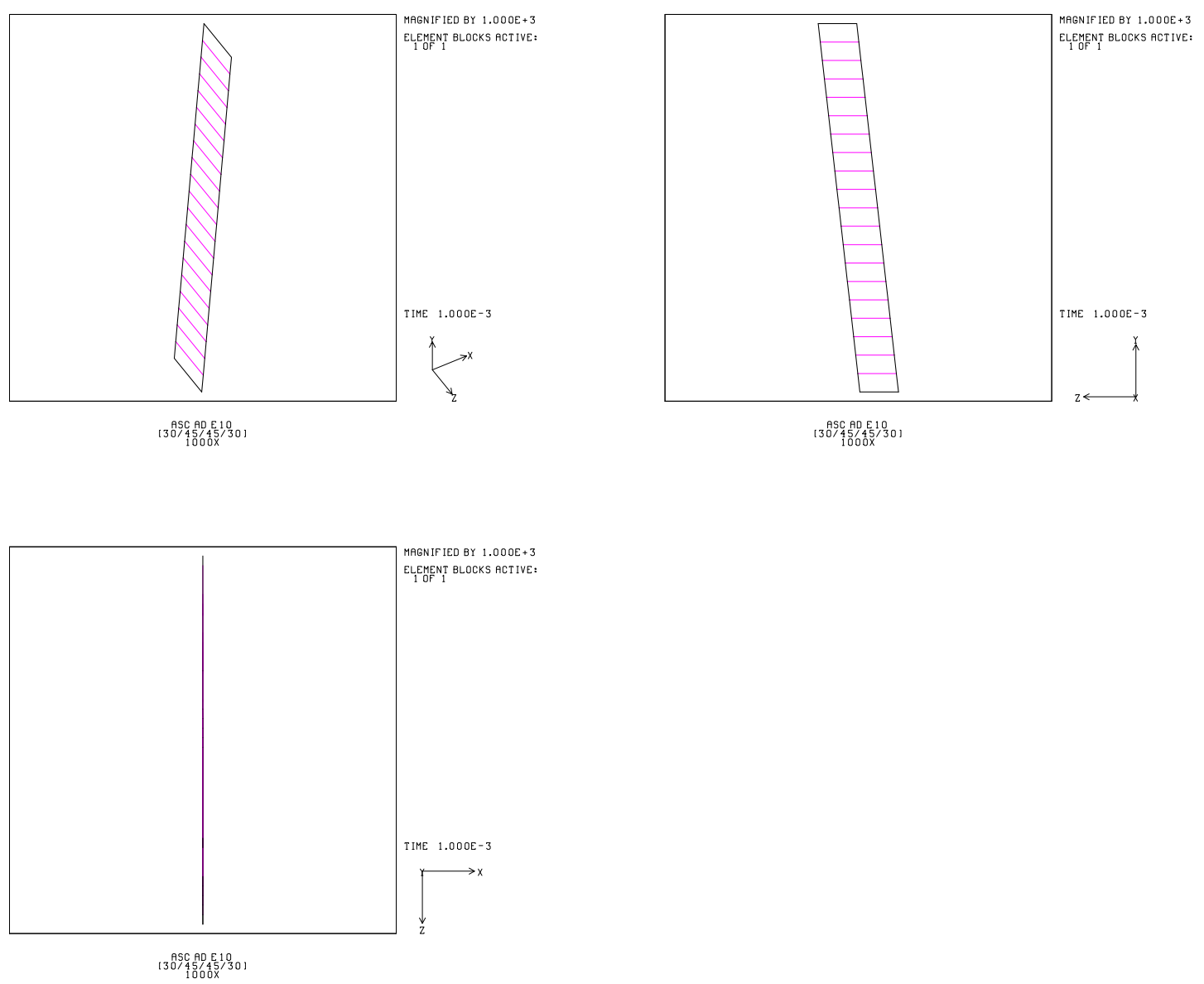

Figure 3.12: Deformed 0.1" high [30/45/45/30] Ring B shell model showing 1 element in circumferential direction. Deformations magnified by 1000 . 
Table 3.25: Radial Displacement at middle of inside surface for 0.01" high [30/45/30/30] Ring B.

\begin{tabular}{ll} 
Case & $\begin{array}{l}V_{r} \\
\text { (in.) }\end{array}$ \\
\hline \hline Cont. FEM & 0.00224834 \\
Shell FEM & 0.00202239 \\
\hline \hline
\end{tabular}

Table 3.26: Stress $\sigma_{X X}=\sigma_{r r}$ (average over layer) for 0.01" high [30/45/30/30] Ring B.

\begin{tabular}{lllll} 
Case & $\begin{array}{l}\text { L1 } \\
(\mathrm{psi})\end{array}$ & $\begin{array}{l}\text { L2 } \\
(\mathrm{psi})\end{array}$ & $\begin{array}{l}\text { L3 } \\
(\mathrm{psi})\end{array}$ & $\begin{array}{l}\text { L4 } \\
\text { (psi) }\end{array}$ \\
\hline \hline Cont. FEM & $-1.366517 \mathrm{E}-01$ & $-3.938791 \mathrm{E}-01$ & $-6.084564 \mathrm{E}-01$ & $-8.641918 \mathrm{E}-01$ \\
Shell FEM & $2.517050 \mathrm{E}-09$ & $2.515813 \mathrm{E}-09$ & $2.515813 \mathrm{E}-09$ & $2.517050 \mathrm{E}-09$ \\
\hline \hline
\end{tabular}

Table 3.27: Stress $\sigma_{Y Y}=\sigma_{z z}$ (average over layer) for 0.01" high [30/45/30/30] Ring B.

\begin{tabular}{lllll} 
Case & $\begin{array}{l}\text { L1 } \\
(\mathrm{psi})\end{array}$ & $\begin{array}{l}\text { L2 } \\
(\mathrm{psi})\end{array}$ & $\begin{array}{l}\text { L3 } \\
(\mathrm{psi})\end{array}$ & $\begin{array}{l}\text { L4 } \\
(\mathrm{psi})\end{array}$ \\
\hline \hline Cont. FEM & -19.25563 & 19.26583 & 19.28215 & -19.26312 \\
Shell FEM & -83.78129 & 83.78321 & 83.78173 & -83.78320 \\
\hline \hline
\end{tabular}

Table 3.28: Stress $\sigma_{Z Z}=\sigma_{\theta \theta}$ (average over layer) for 0.01" high [30/45/30/30] Ring B.

\begin{tabular}{llllll} 
Case & $\begin{array}{l}\text { L1 } \\
(\mathrm{psi})\end{array}$ & $\begin{array}{l}\text { L2 } \\
(\mathrm{psi})\end{array}$ & $\begin{array}{l}\text { L3 } \\
(\mathrm{psi})\end{array}$ & $\begin{array}{l}\text { L4 } \\
(\mathrm{psi})\end{array}$ & $\begin{array}{l}\text { AVG } \\
(\mathrm{psi})\end{array}$ \\
\hline \hline Cont. FEM & 926.0904 & 655.4124 & 655.6328 & 927.0034 & 791.0348 \\
Shell FEM & 945.3318 & 636.7004 & 636.6885 & 945.2853 & 791.0015 \\
\hline \hline
\end{tabular}


Table 3.29: Stress $\sigma_{X Y}=\sigma_{z r}$ (average over layer) for 0.01" high [30/45/30/30] Ring B.

\begin{tabular}{lllll} 
Case & $\begin{array}{l}\text { L1 } \\
(\mathrm{psi})\end{array}$ & $\begin{array}{l}\text { L2 } \\
(\mathrm{psi})\end{array}$ & $\begin{array}{l}\text { L3 } \\
(\mathrm{psi})\end{array}$ & $\begin{array}{l}\text { L4 } \\
(\mathrm{psi})\end{array}$ \\
\hline \hline Cont. FEM & $-2.939306 \mathrm{E}-03$ & $5.007919 \mathrm{E}-03$ & $1.916127 \mathrm{E}-03$ & $-6.155546 \mathrm{E}-04$ \\
Shell FEM & $-1.529511 \mathrm{E}-05$ & $1.814652 \mathrm{E}-05$ & $1.814592 \mathrm{E}-05$ & $-1.529484 \mathrm{E}-05$ \\
\hline \hline
\end{tabular}

Table 3.30: Stress $\sigma_{Y Z}=-\sigma_{\theta z}$ (average over layer) for 0.01" high [30/45/30/30] Ring B.

\begin{tabular}{lllll} 
Case & $\begin{array}{l}\text { L1 } \\
(\mathrm{psi})\end{array}$ & $\begin{array}{l}\text { L2 } \\
(\mathrm{psi})\end{array}$ & $\begin{array}{l}\text { L3 } \\
(\mathrm{psi})\end{array}$ & $\begin{array}{l}\text { L4 } \\
(\mathrm{psi})\end{array}$ \\
\hline \hline Cont. FEM & -174.1657 & 174.3227 & 174.3401 & -174.3924 \\
Shell FEM & -351.9023 & 351.9046 & 351.8919 & -351.8970 \\
\hline \hline
\end{tabular}

Table 3.31: Stress $\sigma_{Z X}=-\sigma_{r \theta}$ (average over layer) for 0.01" high [30/45/30/30] Ring B.

\begin{tabular}{lllll} 
Case & $\begin{array}{l}\text { L1 } \\
(\mathrm{psi})\end{array}$ & $\begin{array}{l}\text { L2 } \\
(\mathrm{psi})\end{array}$ & $\begin{array}{l}\text { L3 } \\
(\mathrm{psi})\end{array}$ & $\begin{array}{l}\text { L4 } \\
(\mathrm{psi})\end{array}$ \\
\hline \hline Cont. FEM & $1.423775 \mathrm{E}-02$ & $5.067150 \mathrm{E}-02$ & $1.431179 \mathrm{E}-02$ & $6.711946 \mathrm{E}-02$ \\
Shell FEM & $2.623334 \mathrm{E}-02$ & $2.621714 \mathrm{E}-02$ & $2.621714 \mathrm{E}-02$ & $2.623334 \mathrm{E}-02$ \\
\hline \hline
\end{tabular}

Table 3.32: Computational cost for 0.01" high [30/45/30/30] Ring B.

\begin{tabular}{lllllll} 
Case & Model & Elements & Nodes & DOF w/o BC & Iterations & $\begin{array}{l}\text { CPU } \\
\text { (sec) }\end{array}$ \\
\hline \hline Cont. FEM & 0.0625 deg & 576 & 875 & 2625 & 1336 & 12.450 \\
Shell FEM & $0.0625 \mathrm{deg}$ & 24 & 35 & 210 & 742 & 1.099 \\
\hline \hline
\end{tabular}



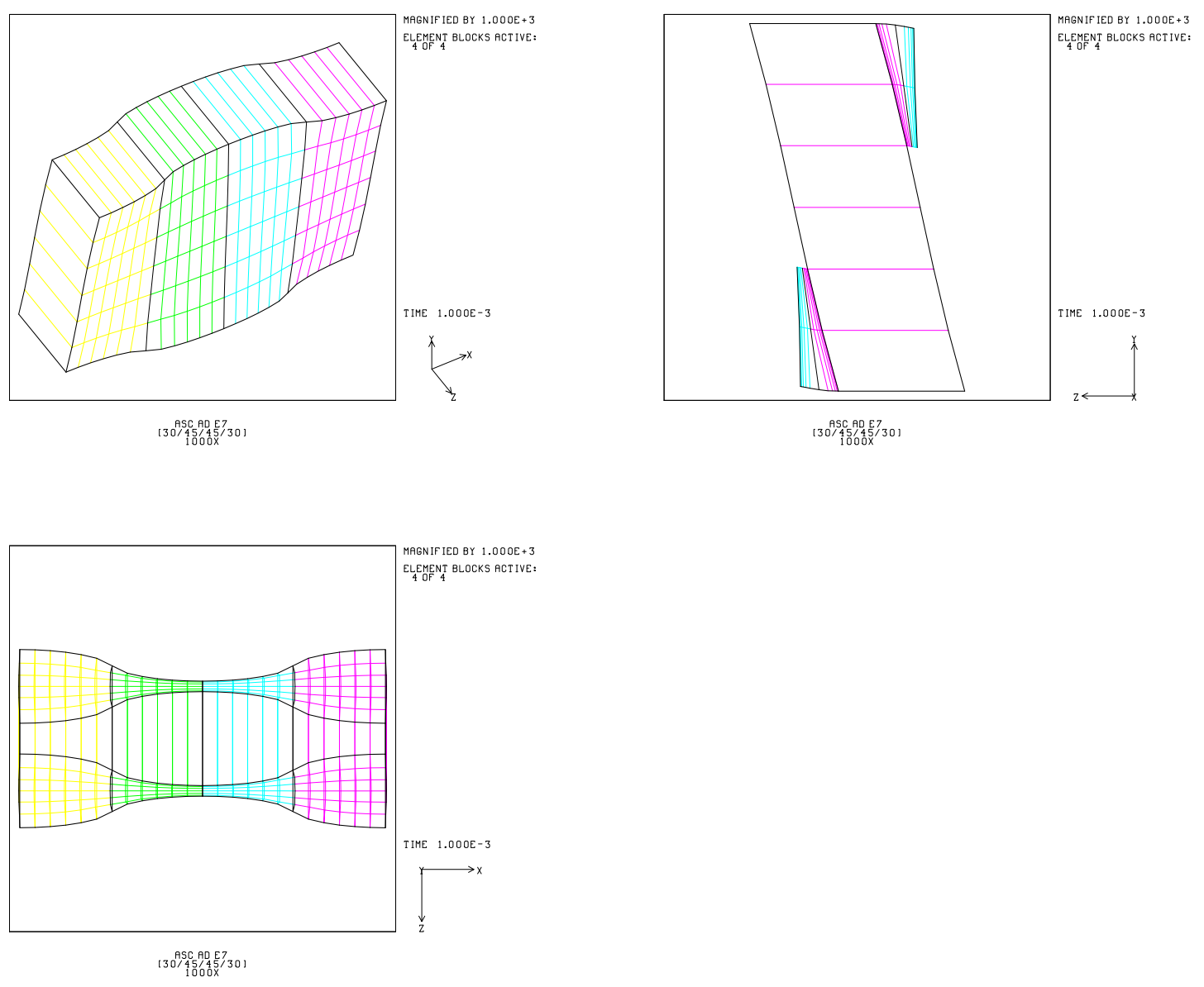

Figure 3.13: Deformed 0.01" high [30/45/45/30] Ring B continuum model showing 1 element in circumferential direction. Deformations magnified by 1000 . 
(a)

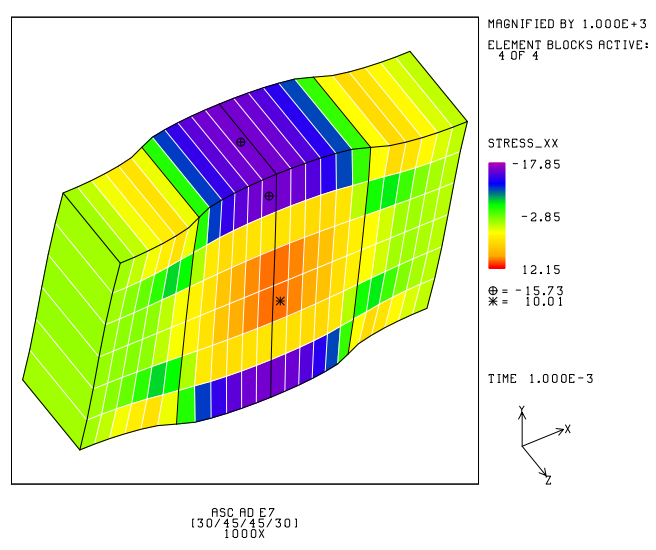

(c)

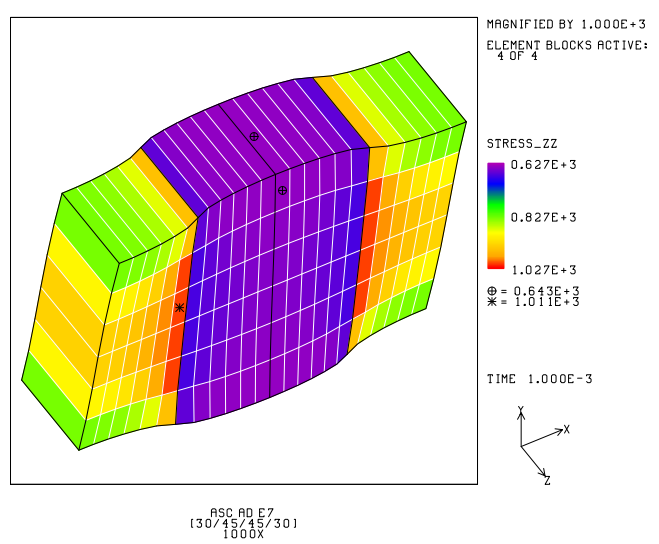

(b)

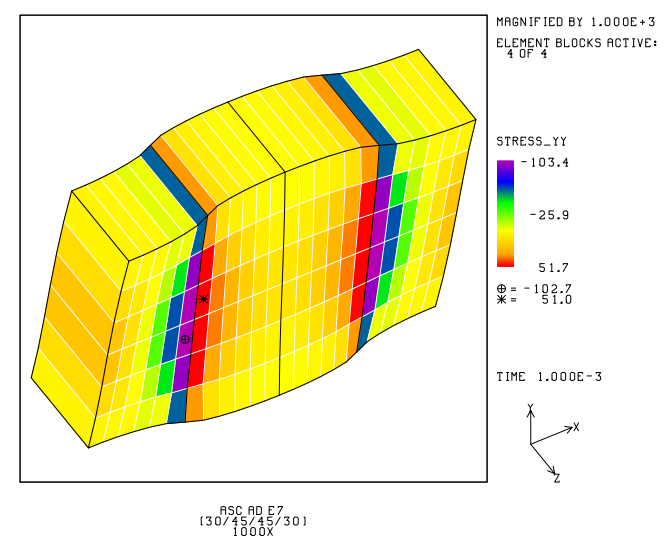

Figure 3.14: Stress distribution for deformed 0.01" high [30/45/45/30] Ring B continuum model: (a) $\sigma_{X X}=\sigma_{r r}$; (b) $\sigma_{Y Y}=\sigma_{z z}$; (c) $\sigma_{Z Z}=\sigma_{\theta \theta}$. Deformations magnified by 1000 . 
(a)

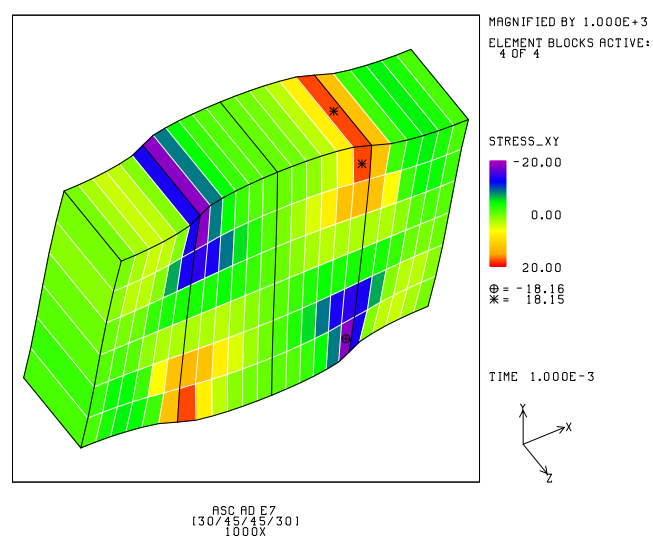

(c)

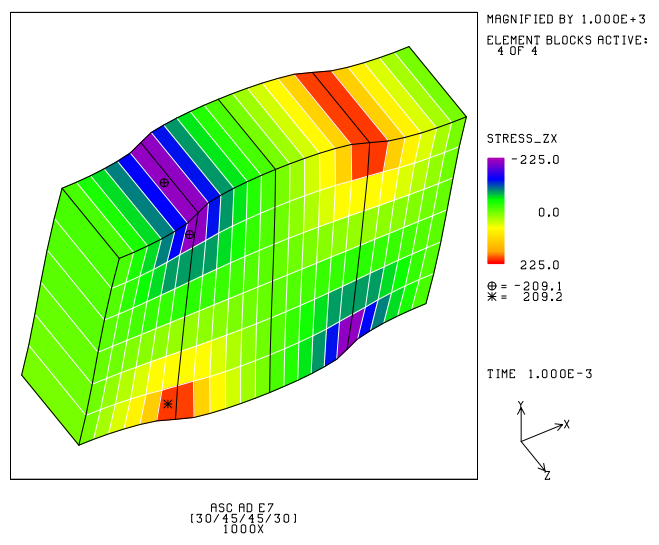

(b)

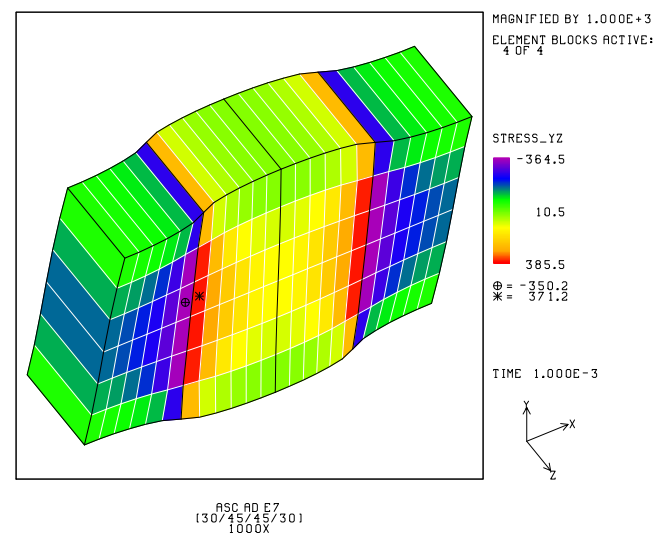

Figure 3.15: Stress distribution for deformed 0.01" high [30/45/45/30] Ring B continuum model: (a) $\sigma_{X Y}=\sigma_{z r}$; (b) $\sigma_{Y Z}=-\sigma_{\theta z}$; (c) $\sigma_{Z X}=-\sigma_{r \theta}$. Deformations magnified by 1000 . 

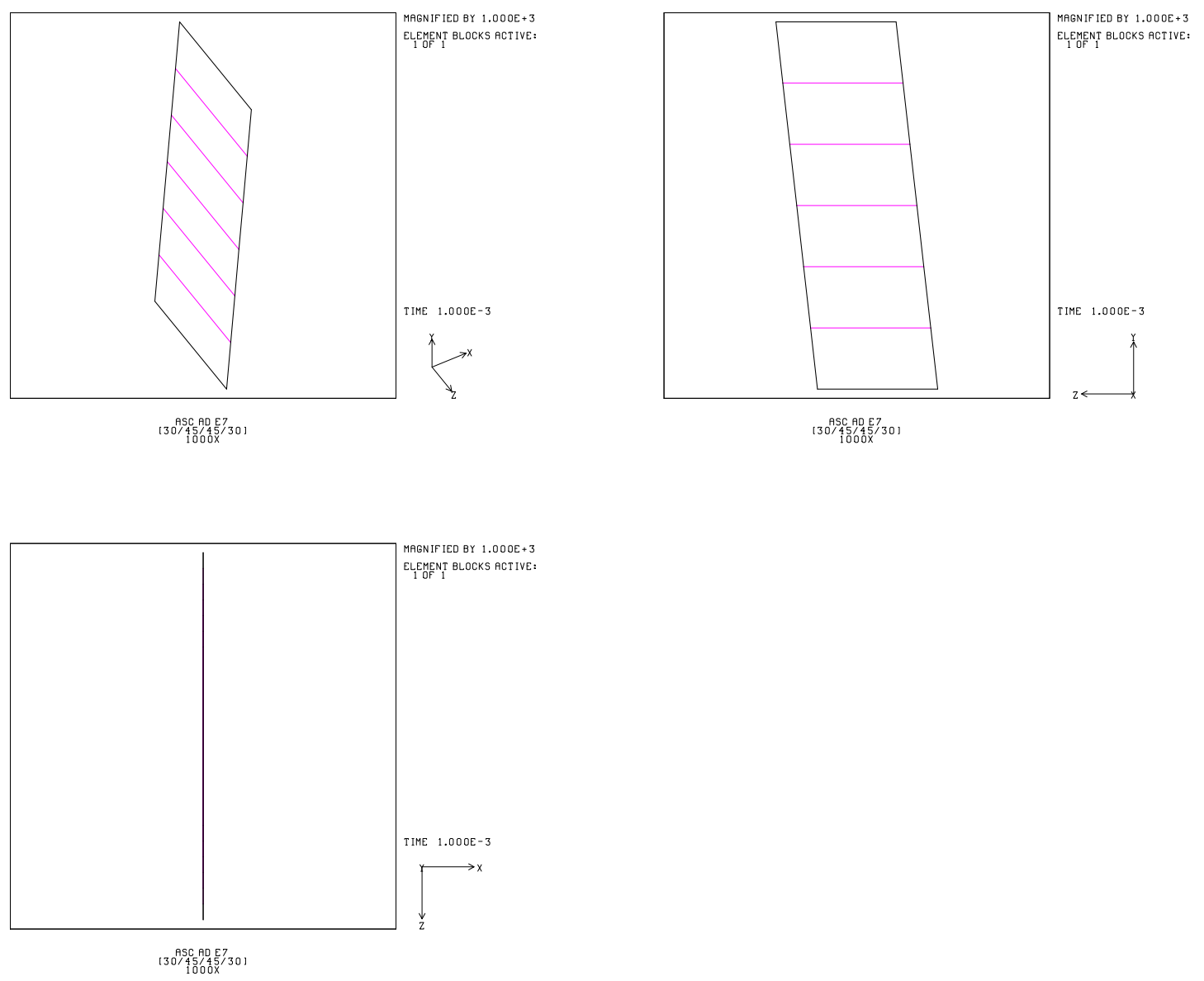

Figure 3.16: Deformed 0.01" high [30/45/45/30] Ring B shell model showing 1 element in circumferential direction. Deformations magnified by 1000 . 

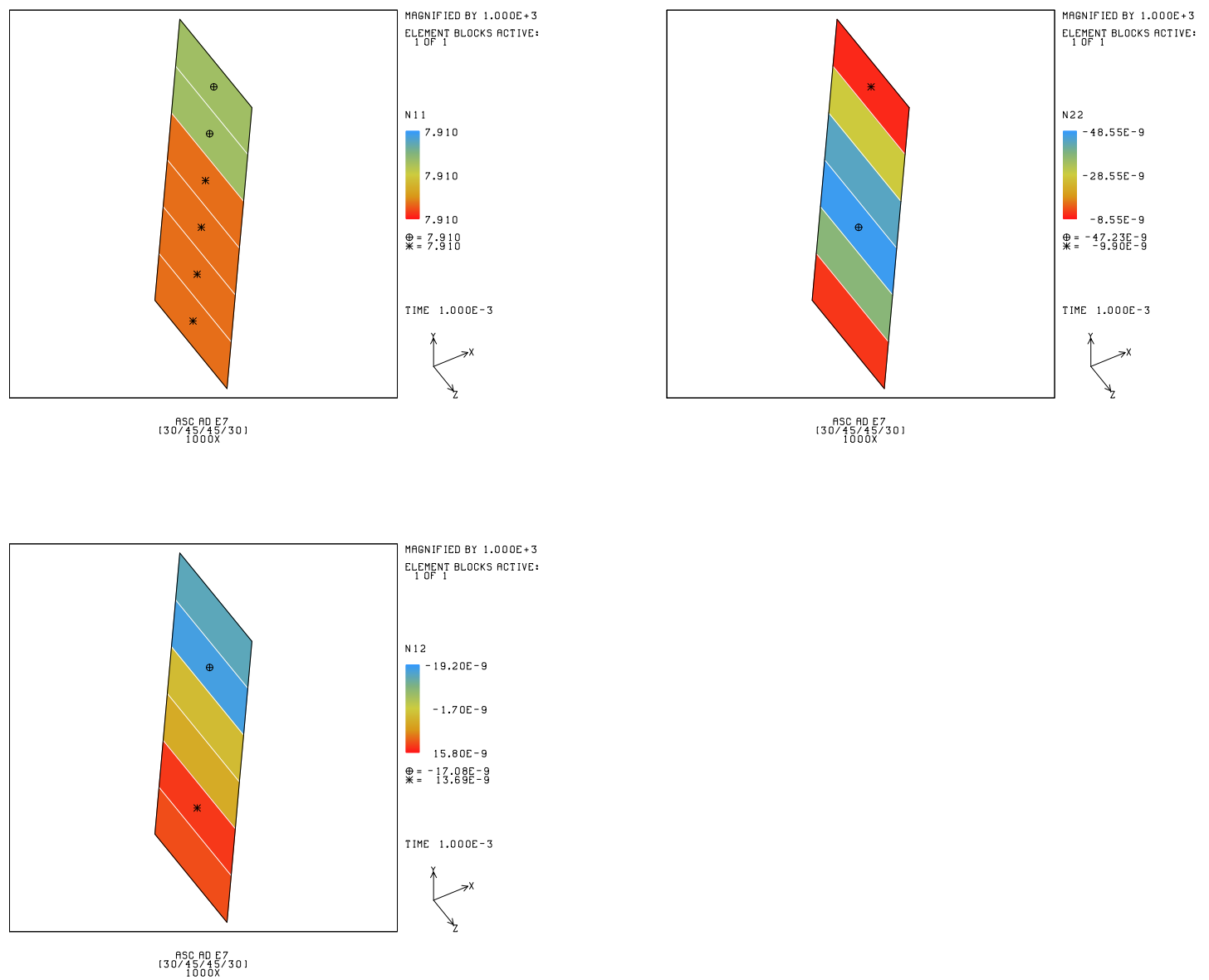

Figure 3.17: Force resultants distribution for deformed 0.01" high [30/45/45/30] Ring B shell model: (a) $N_{11}=N_{\theta \theta}$; (b) $N_{22}=N_{z z}$; (c) $N_{12}=N_{\theta z}$. Deformations magnified by 1000 . 

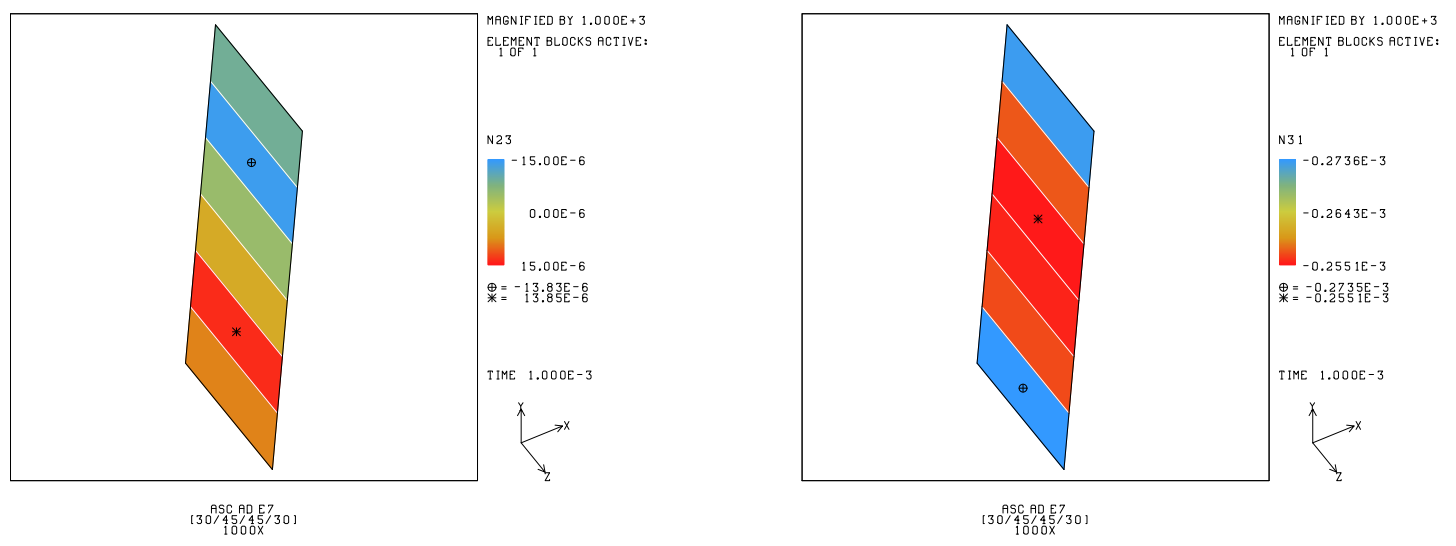

Figure 3.18: Force resultants distribution for deformed 0.01" high [30/45/45/30] Ring B shell model: (a) $N_{23}=N_{z r}$; (b) $N_{31}=N_{r \theta}$. Deformations magnified by 1000 . 

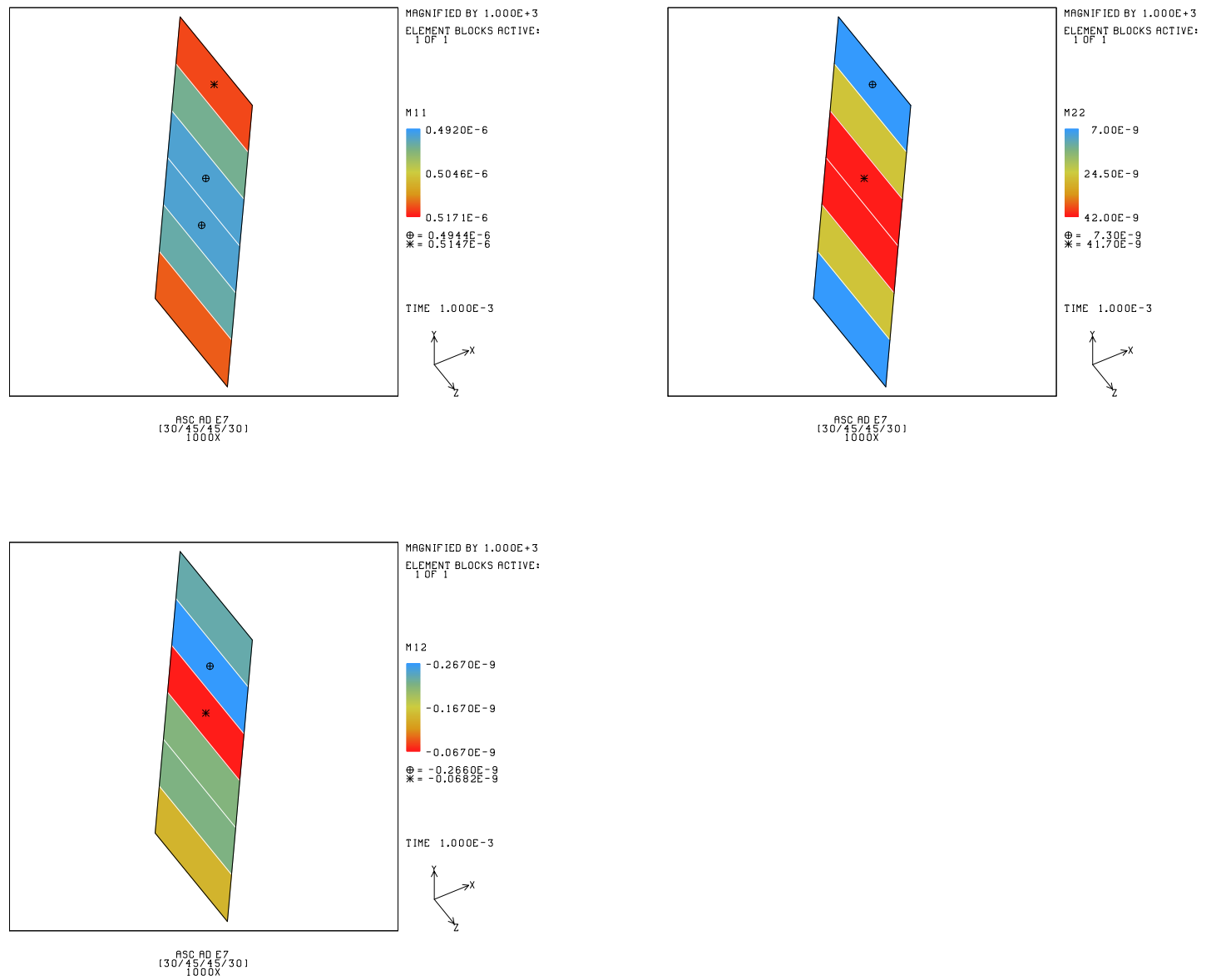

Figure 3.19: Force-couple resultants distribution for deformed 0.01" high [30/45/45/30] Ring B shell model: (a) $M_{11}=M_{\theta \theta}$; (b) $M_{22}=M_{z z}$; (c) $M_{12}=M_{\theta z}$. Deformations magnified by 1000 . 
Table 3.33: Radial displacement at middle of inside surface for [30/-30/30/-30] Ring B.

\begin{tabular}{ll} 
Case & $\begin{array}{l}V_{r} \\
\text { (in. })\end{array}$ \\
\hline \hline Cont. FEM & 0.00133504 \\
Shell FEM & 0.00141851 \\
\hline \hline
\end{tabular}

Table 3.34: Stress $\sigma_{X X}=\sigma_{r r}$ at ring center for [30/-30/30/-30] Ring B.

\begin{tabular}{lllll} 
Case & $\begin{array}{l}\text { L1 } \\
(\mathrm{psi})\end{array}$ & $\begin{array}{l}\text { L2 } \\
(\mathrm{psi})\end{array}$ & $\begin{array}{l}\text { L3 } \\
(\mathrm{psi})\end{array}$ & $\begin{array}{l}\text { L4 } \\
(\mathrm{psi})\end{array}$ \\
\hline \hline Cont. FEM & $-1.234314 \mathrm{E}-01$ & $-3.684242 \mathrm{E}-01$ & $-6.204792 \mathrm{E}-01$ & $-8.742052 \mathrm{E}-01$ \\
Shell FEM & $1.607640 \mathrm{E}-09$ & $1.607727 \mathrm{E}-09$ & $1.607723 \mathrm{E}-09$ & $1.607641 \mathrm{E}-09$ \\
\hline \hline
\end{tabular}

Table 3.35: Stress $\sigma_{Y Y}=\sigma_{z z}$ at ring center for [30/-30/30/-30] Ring B.

\begin{tabular}{lllll} 
Case & $\begin{array}{l}\text { L1 } \\
(\mathrm{psi})\end{array}$ & $\begin{array}{l}\text { L2 } \\
(\mathrm{psi})\end{array}$ & $\begin{array}{l}\text { L3 } \\
(\mathrm{psi})\end{array}$ & $\begin{array}{l}\text { L4 } \\
(\mathrm{psi})\end{array}$ \\
\hline \hline Cont. FEM & $-5.058408 \mathrm{E}-01$ & $-3.142714 \mathrm{E}-01$ & $1.706637 \mathrm{E}-01$ & $6.368898 \mathrm{E}-01$ \\
Shell FEM & 61.22946 & -61.16712 & -61.18947 & 61.13670 \\
\hline \hline
\end{tabular}

Table 3.36: Stress $\sigma_{Z Z}=\sigma_{\theta \theta}$ at ring center for [30/-30/30/-30] Ring B.

\begin{tabular}{llllll} 
Case & $\begin{array}{l}\text { L1 } \\
(\mathrm{psi})\end{array}$ & $\begin{array}{l}\text { L2 } \\
(\mathrm{psi})\end{array}$ & $\begin{array}{l}\text { L3 } \\
(\mathrm{psi})\end{array}$ & $\begin{array}{l}\text { L4 } \\
(\mathrm{psi})\end{array}$ & $\begin{array}{l}\text { AVG } \\
(\mathrm{psi})\end{array}$ \\
\hline \hline Cont. FEM & 800.2952 & 801.0306 & 801.3671 & 801.7778 & 801.1177 \\
Shell FEM & 702.3005 & 879.7058 & 879.6369 & 702.1315 & 790.9437 \\
\hline \hline
\end{tabular}

Table 3.37: Stress $\sigma_{X Y}=\sigma_{z r}$ at ring center for [30/-30/30/-30] Ring B.

\begin{tabular}{lllll} 
Case & $\begin{array}{l}\text { L1 } \\
(\mathrm{psi})\end{array}$ & $\begin{array}{l}\text { L2 } \\
(\mathrm{psi})\end{array}$ & $\begin{array}{l}\text { L3 } \\
(\mathrm{psi})\end{array}$ & $\begin{array}{l}\text { L4 } \\
(\mathrm{psi})\end{array}$ \\
\hline \hline Cont. FEM & $2.037678 \mathrm{E}-03$ & $1.994841 \mathrm{E}-02$ & $-1.885386 \mathrm{E}-02$ & $7.482109 \mathrm{E}-03$ \\
Shell FEM & $-9.842990 \mathrm{E}-05$ & $-1.138081 \mathrm{E}-04$ & $-9.132145 \mathrm{E}-05$ & $-1.067193 \mathrm{E}-04$ \\
\hline \hline
\end{tabular}


Table 3.38: Stress $\sigma_{Y Z}=-\sigma_{\theta z}$ at ring center for [30/-30/30/-30] Ring B.

\begin{tabular}{lllll} 
Case & $\begin{array}{l}\text { L1 } \\
(\mathrm{psi})\end{array}$ & $\begin{array}{l}\text { L2 } \\
(\mathrm{psi})\end{array}$ & $\begin{array}{l}\text { L3 } \\
(\mathrm{psi})\end{array}$ & $\begin{array}{l}\text { L4 } \\
(\mathrm{psi})\end{array}$ \\
\hline \hline Cont. FEM & -402.2333 & 402.7932 & -402.6165 & 402.5658 \\
Shell FEM & -190.1512 & 515.7996 & -515.7662 & 190.1199 \\
\hline
\end{tabular}

Table 3.39: Stress $\sigma_{Z X}=-\sigma_{r \theta}$ at ring center for [30/-30/30/-30] Ring B.

\begin{tabular}{lllll} 
Case & $\begin{array}{l}\text { L1 } \\
(\mathrm{psi})\end{array}$ & $\begin{array}{l}\mathrm{L} 2 \\
(\mathrm{psi})\end{array}$ & $\begin{array}{l}\text { L3 } \\
(\mathrm{psi})\end{array}$ & $\begin{array}{l}\text { L4 } \\
(\mathrm{psi})\end{array}$ \\
\hline \hline Cont. FEM & $1.904779 \mathrm{E}-02$ & $2.953888 \mathrm{E}-02$ & $3.683479 \mathrm{E}-02$ & $2.801812 \mathrm{E}-02$ \\
Shell FEM & $-3.688214 \mathrm{E}-02$ & $-3.688607 \mathrm{E}-02$ & $-3.688598 \mathrm{E}-02$ & $-3.688217 \mathrm{E}-02$ \\
\hline \hline
\end{tabular}

Table 3.40: Computational cost for [30/-30/30/-30] Ring B.

\begin{tabular}{lllllll} 
Case & Model & Elements & Nodes & DOF w/o BC & Iterations & $\begin{array}{l}\text { CPU } \\
\text { (sec) }\end{array}$ \\
\hline \hline Cont. FEM & $0.5 \mathrm{deg}$ & 800 & 1155 & 3465 & 947 & 12.589 \\
Shell FEM & $0.5 \mathrm{deg}$ & 200 & 231 & 1386 & 1961 & 13.419 \\
\hline \hline
\end{tabular}



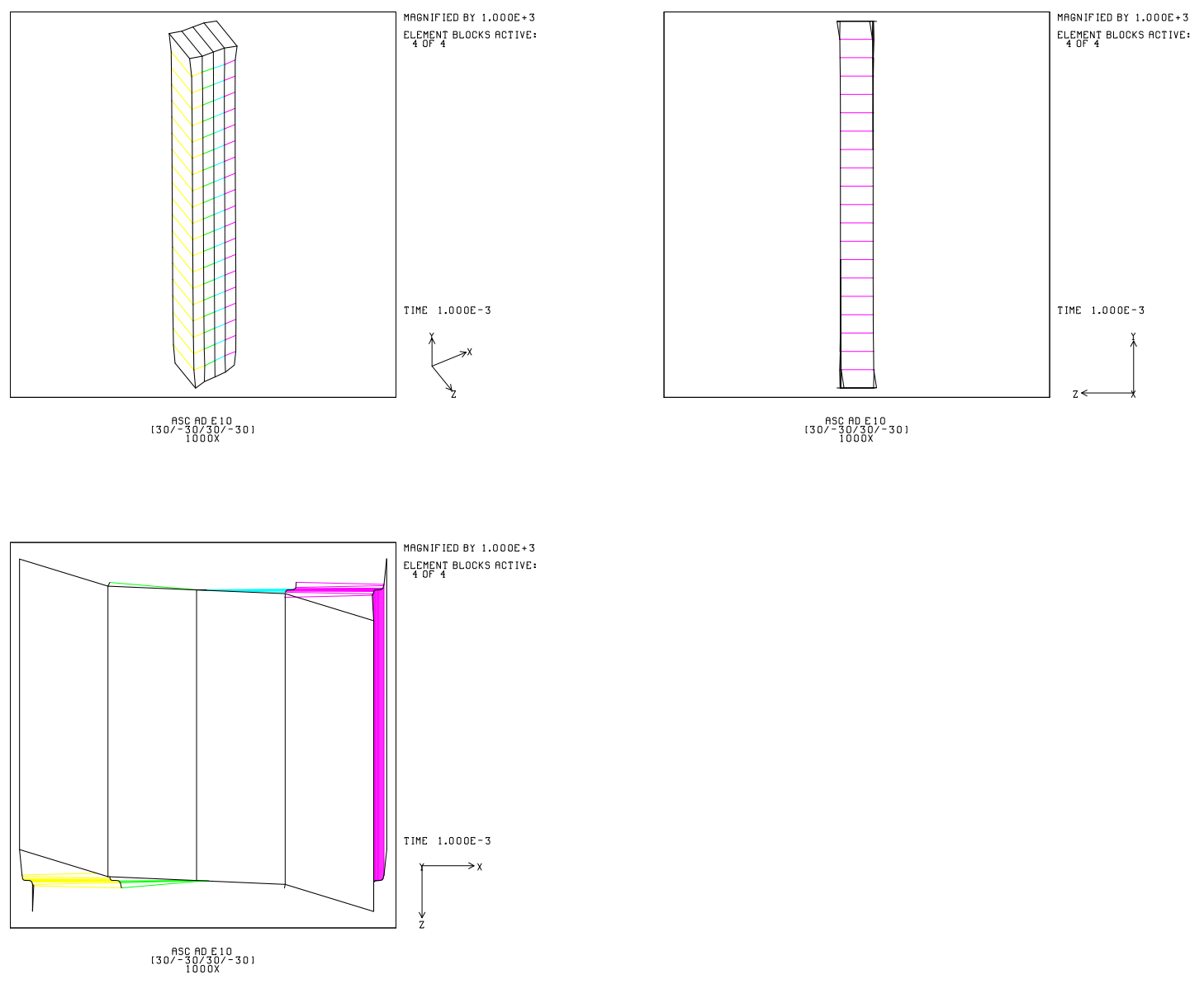

Figure 3.20: Deformed [30/-30/30/-30] Ring B continuum model showing 1 element in circumferential direction. Deformations magnified by 1000. 

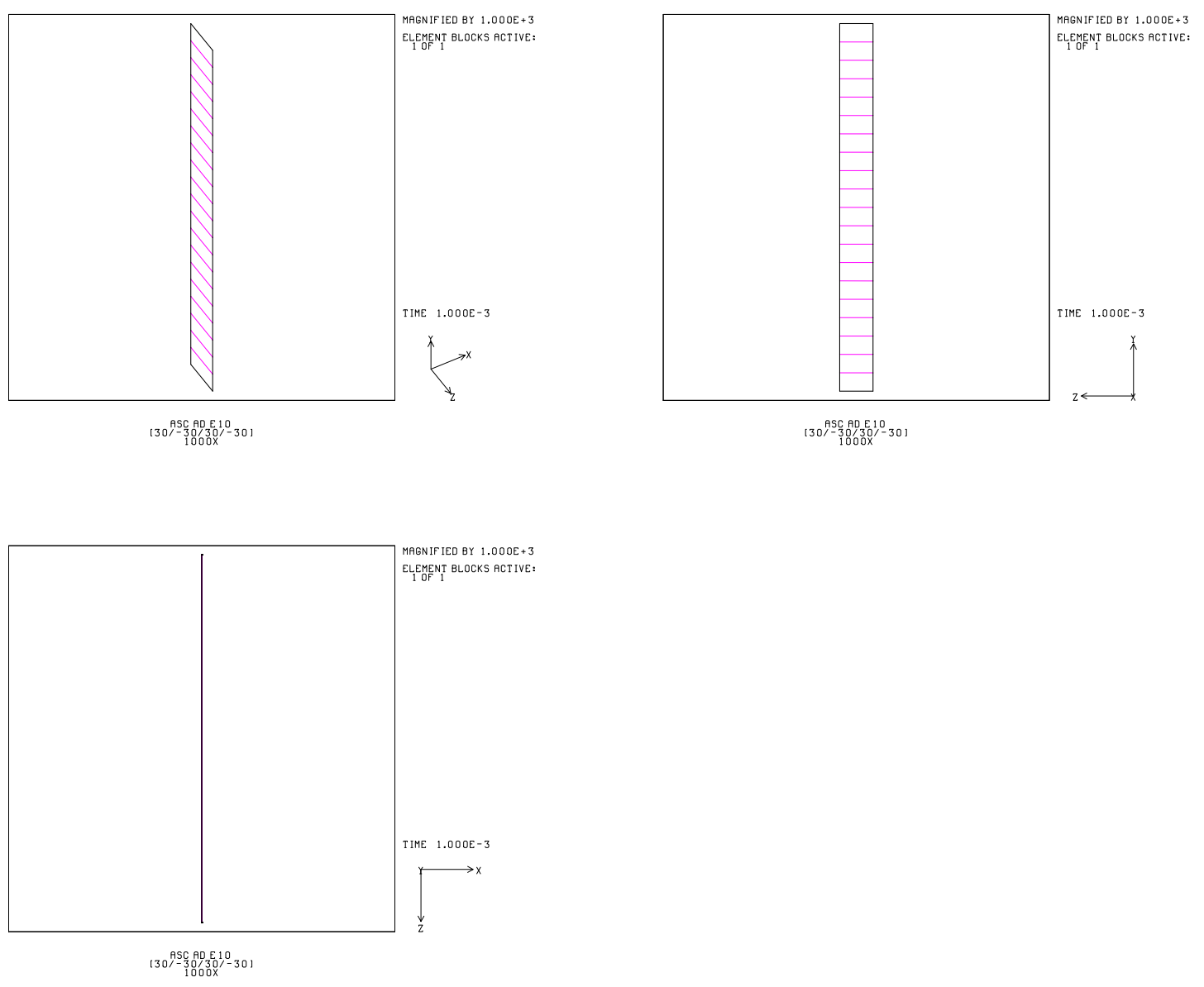

Figure 3.21: Deformed [30/-30/30/-30] Ring B shell model showing 1 element in circumferential direction. Deformations magnified by 1000 . 

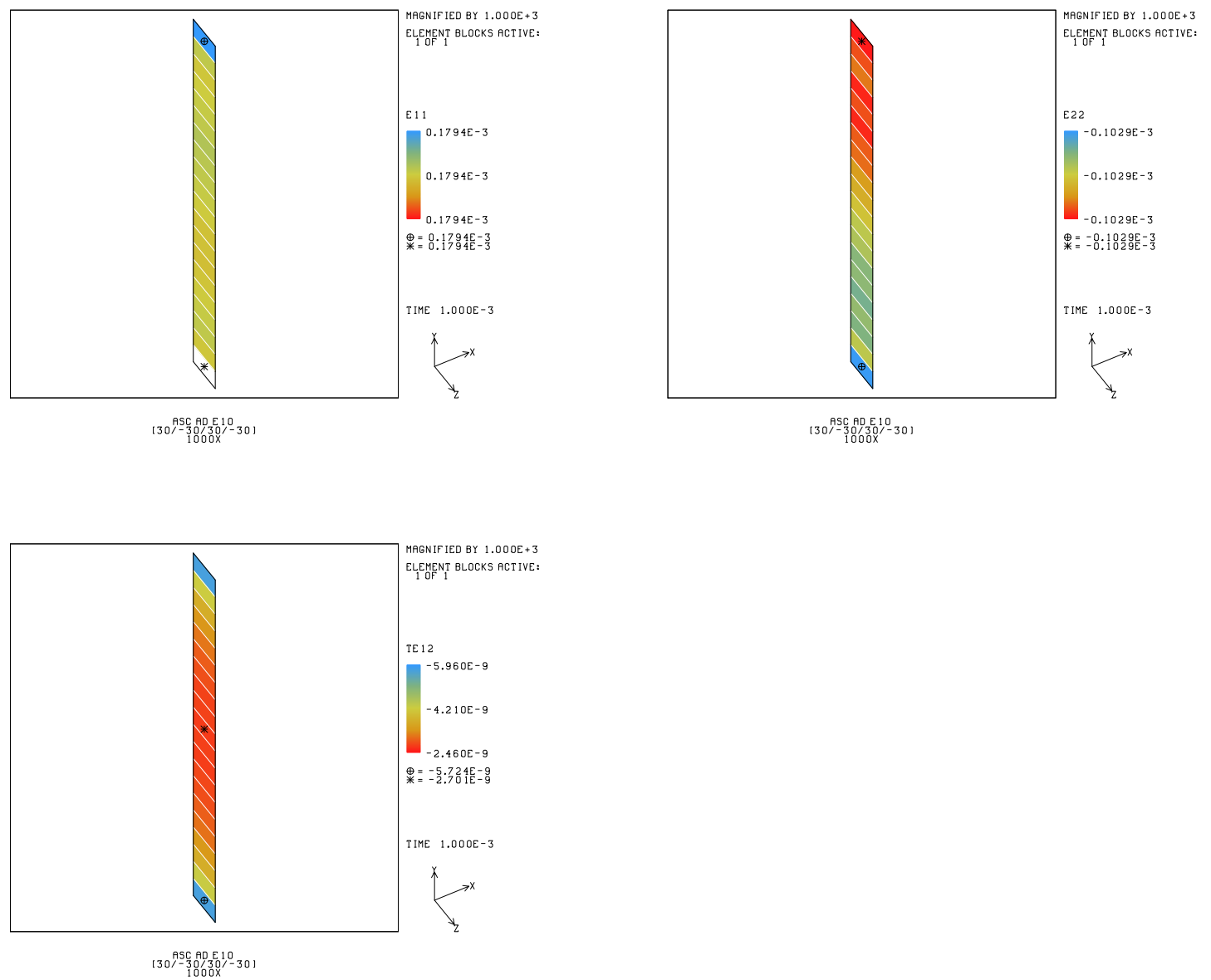

Figure 3.22: Inplane membrane strain distribution for deformed [30/-30/30/-30] Ring B shell model: (a) $e_{11}=e_{\theta \theta}$; (b) $e_{22}=e_{z z}$; (c) $2 e_{12}=2 e_{\theta z}$. Deformations magnified by 1000 . 

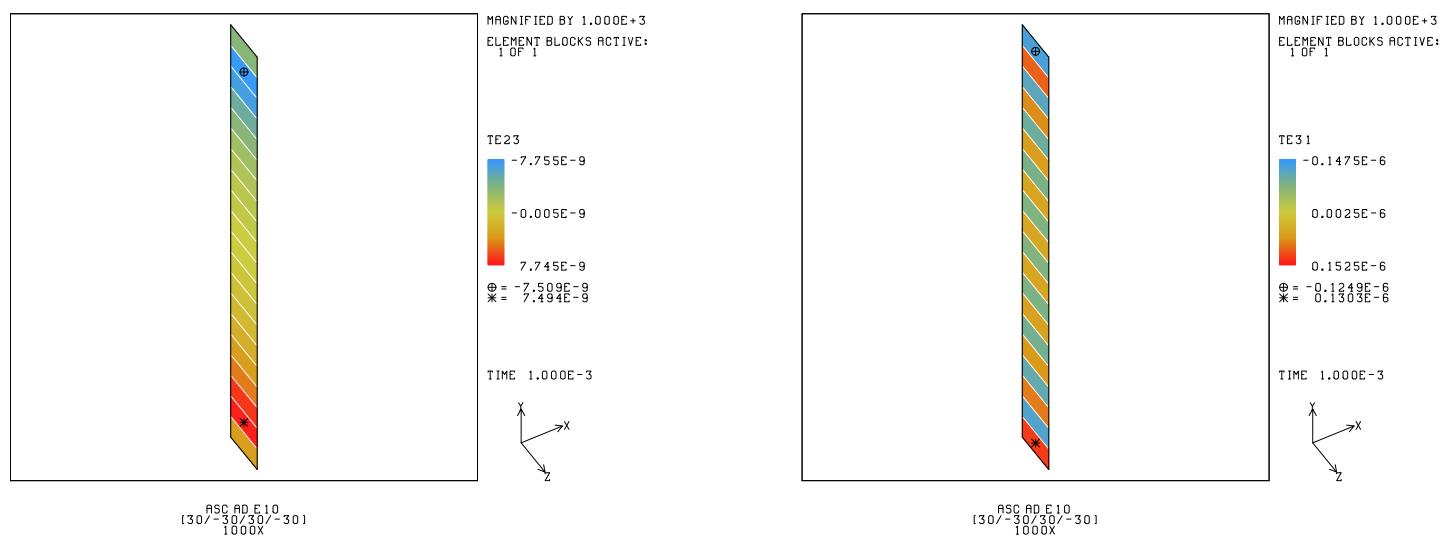

Figure 3.23: Transverse shear strain distribution for deformed [30/-30/30/-30] Ring B shell model: (a) $2 e_{23}=2 e_{z r}$; (b) $2 e_{31}=2 e_{r \theta}$. Deformations magnified by 1000 . 

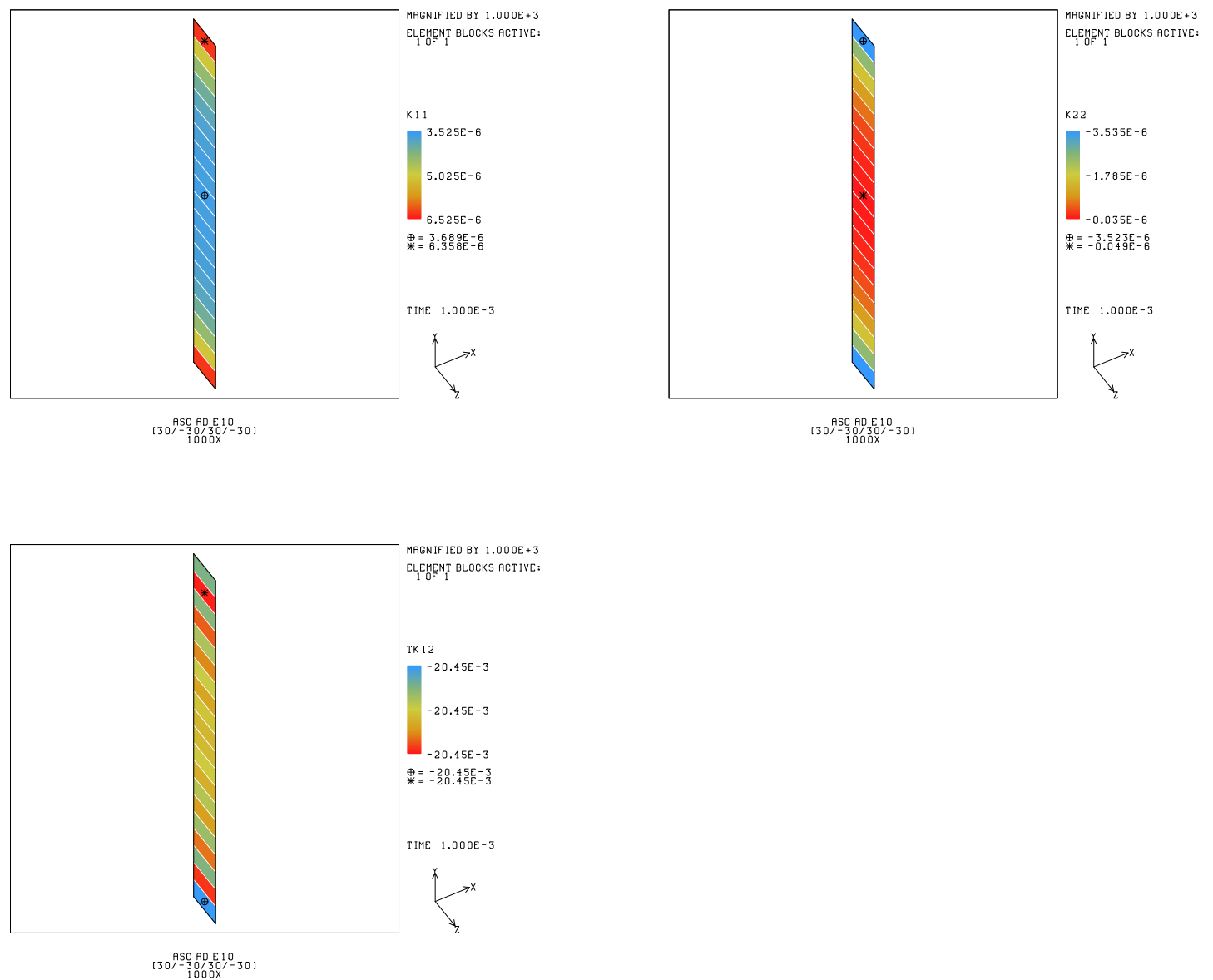

Figure 3.24: Bending strain distribution for deformed [30/-30/30/-30] Ring B shell model: (a) $\kappa_{11}=\kappa_{\theta \theta}$; (b) $\kappa_{22}=\kappa_{z z}$; (c) $2 \kappa_{12}=2 \kappa_{\theta z}$. Deformations magnified by 1000. 
Table 3.41: Radial Displacement at middle of inside surface for [30/60/50/10] Ring B.

\begin{tabular}{ll} 
Case & $\begin{array}{l}V_{r} \\
\text { (in. })\end{array}$ \\
\hline \hline Cont. FEM & 0.00120676 \\
Shell FEM & 0.00119091 \\
\hline \hline
\end{tabular}

Table 3.42: Stress $\sigma_{X X}=\sigma_{r r}$ at ring center for [30/60/50/10] Ring B.

\begin{tabular}{lllll} 
Case & $\begin{array}{l}\text { L1 } \\
(\mathrm{psi})\end{array}$ & $\begin{array}{l}\text { L2 } \\
(\mathrm{psi})\end{array}$ & $\begin{array}{l}\text { L3 } \\
(\mathrm{psi})\end{array}$ & $\begin{array}{l}\text { L4 } \\
(\mathrm{psi})\end{array}$ \\
\hline \hline Cont. FEM & $-1.056251 \mathrm{E}-01$ & $-3.155246 \mathrm{E}-01$ & $-5.124476 \mathrm{E}-01$ & $-8.027225 \mathrm{E}-01$ \\
Shell FEM & $-3.145848 \mathrm{E}-08$ & $-3.403898 \mathrm{E}-08$ & $4.433894 \mathrm{E}-08$ & $-6.402386 \mathrm{E}-08$ \\
\hline \hline
\end{tabular}

Table 3.43: Stress $\sigma_{Y Y}=\sigma_{z z}$ at ring center for [30/60/50/10] Ring B.

\begin{tabular}{lllll} 
Case & $\begin{array}{l}\text { L1 } \\
(\mathrm{psi})\end{array}$ & $\begin{array}{l}\text { L2 } \\
(\mathrm{psi})\end{array}$ & $\begin{array}{l}\text { L3 } \\
(\mathrm{psi})\end{array}$ & $\begin{array}{l}\text { L4 } \\
(\mathrm{psi})\end{array}$ \\
\hline \hline Cont. FEM & -48.17365 & -45.07275 & 251.1764 & -157.8839 \\
Shell FEM & -39.88367 & -47.78226 & 252.3777 & -164.6430 \\
\hline \hline
\end{tabular}

Table 3.44: Stress $\sigma_{Z Z}=\sigma_{\theta \theta}$ at ring center for [30/60/50/10] Ring B.

\begin{tabular}{llllll} 
Case & $\begin{array}{l}\text { L1 } \\
(\mathrm{psi})\end{array}$ & $\begin{array}{l}\text { L2 } \\
(\mathrm{psi})\end{array}$ & $\begin{array}{l}\text { L3 } \\
(\mathrm{psi})\end{array}$ & $\begin{array}{l}\text { L4 } \\
(\mathrm{psi})\end{array}$ & $\begin{array}{l}\text { AVG } \\
(\mathrm{psi})\end{array}$ \\
\hline \hline Cont. FEM & 657.5120 & 666.9631 & 578.9243 & 1292.930 & 799.0824 \\
Shell FEM & 635.8290 & 660.3647 & 570.6384 & 1289.375 & 789.0518 \\
\hline \hline
\end{tabular}

Table 3.45: Stress $\sigma_{X Y}=\sigma_{z r}$ at ring center for [30/60/50/10] Ring B.

\begin{tabular}{lllll} 
Case & $\begin{array}{l}\text { L1 } \\
(\mathrm{psi})\end{array}$ & $\begin{array}{l}\text { L2 } \\
(\mathrm{psi})\end{array}$ & $\begin{array}{l}\text { L3 } \\
(\mathrm{psi})\end{array}$ & \multicolumn{1}{l}{$\begin{array}{l}\text { L4 } \\
(\mathrm{psi})\end{array}$} \\
\hline \hline Cont. FEM & $-5.734813 \mathrm{E}-03$ & $8.671496 \mathrm{E}-03$ & $1.301813 \mathrm{E}-02$ & $-3.997555 \mathrm{E}-03$ \\
Shell FEM & $-1.289170 \mathrm{E}-03$ & $-1.432853 \mathrm{E}-03$ & $3.417504 \mathrm{E}-03$ & $-3.304830 \mathrm{E}-03$ \\
\hline \hline
\end{tabular}


Table 3.46: Stress $\sigma_{Y Z}=-\sigma_{\theta z}$ at ring center for [30/60/50/10] Ring B.

\begin{tabular}{lllll} 
Case & $\begin{array}{l}\text { L1 } \\
(\mathrm{psi})\end{array}$ & $\begin{array}{l}\text { L2 } \\
(\mathrm{psi})\end{array}$ & $\begin{array}{l}\text { L3 } \\
(\mathrm{psi})\end{array}$ & $\begin{array}{l}\text { L4 } \\
(\mathrm{psi})\end{array}$ \\
\hline \hline Cont. FEM & -324.1679 & 370.4191 & 161.0073 & -207.2136 \\
Shell FEM & -296.3977 & 370.9121 & 140.9399 & -215.4537 \\
\hline
\end{tabular}

Table 3.47: Stress $\sigma_{Z X}=-\sigma_{r \theta}$ at ring center for [30/60/50/10] Ring B.

\begin{tabular}{lllll} 
Case & $\begin{array}{l}\text { L1 } \\
(\mathrm{psi})\end{array}$ & $\begin{array}{l}\mathrm{L} 2 \\
(\mathrm{psi})\end{array}$ & $\begin{array}{l}\text { L3 } \\
(\mathrm{psi})\end{array}$ & $\begin{array}{l}\mathrm{L} 4 \\
(\mathrm{psi})\end{array}$ \\
\hline \hline Cont. FEM & $-9.231838 \mathrm{E}-03$ & $-3.510736 \mathrm{E}-03$ & $-7.435843 \mathrm{E}-04$ & $2.587843 \mathrm{E}-02$ \\
Shell FEM & $7.039402 \mathrm{E}-06$ & $1.077726 \mathrm{E}-02$ & $7.067532 \mathrm{E}-03$ & $1.297668 \mathrm{E}-03$ \\
\hline \hline
\end{tabular}

Table 3.48: Computational cost for [30/60/50/10] Ring B.

\begin{tabular}{lllllll} 
Case & Model & Elements & Nodes & DOF w/o BC & Iterations & $\begin{array}{l}\text { CPU } \\
(\mathrm{sec})\end{array}$ \\
\hline \hline Cont. FEM & $0.5 \mathrm{deg}$ & 800 & 1155 & 3465 & 1341 & 17.459 \\
Shell FEM & $0.5 \mathrm{deg}$ & 200 & 231 & 1386 & 50,173 & 325.040 \\
\hline \hline
\end{tabular}



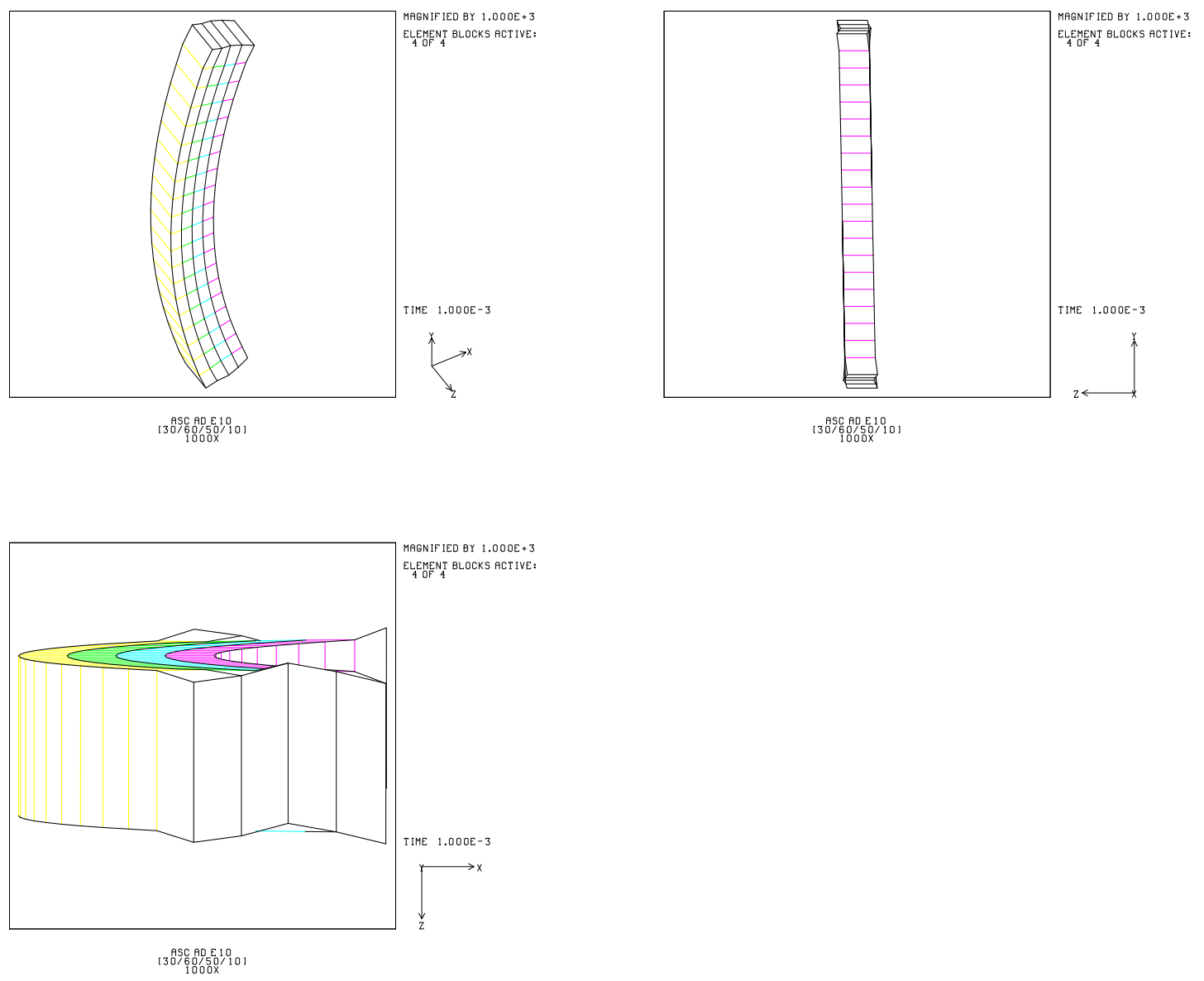

Figure 3.25: Deformed [30/60/50/10] Ring B continuum model showing 1 element in circumferential direction. Deformations magnified by 1000. 

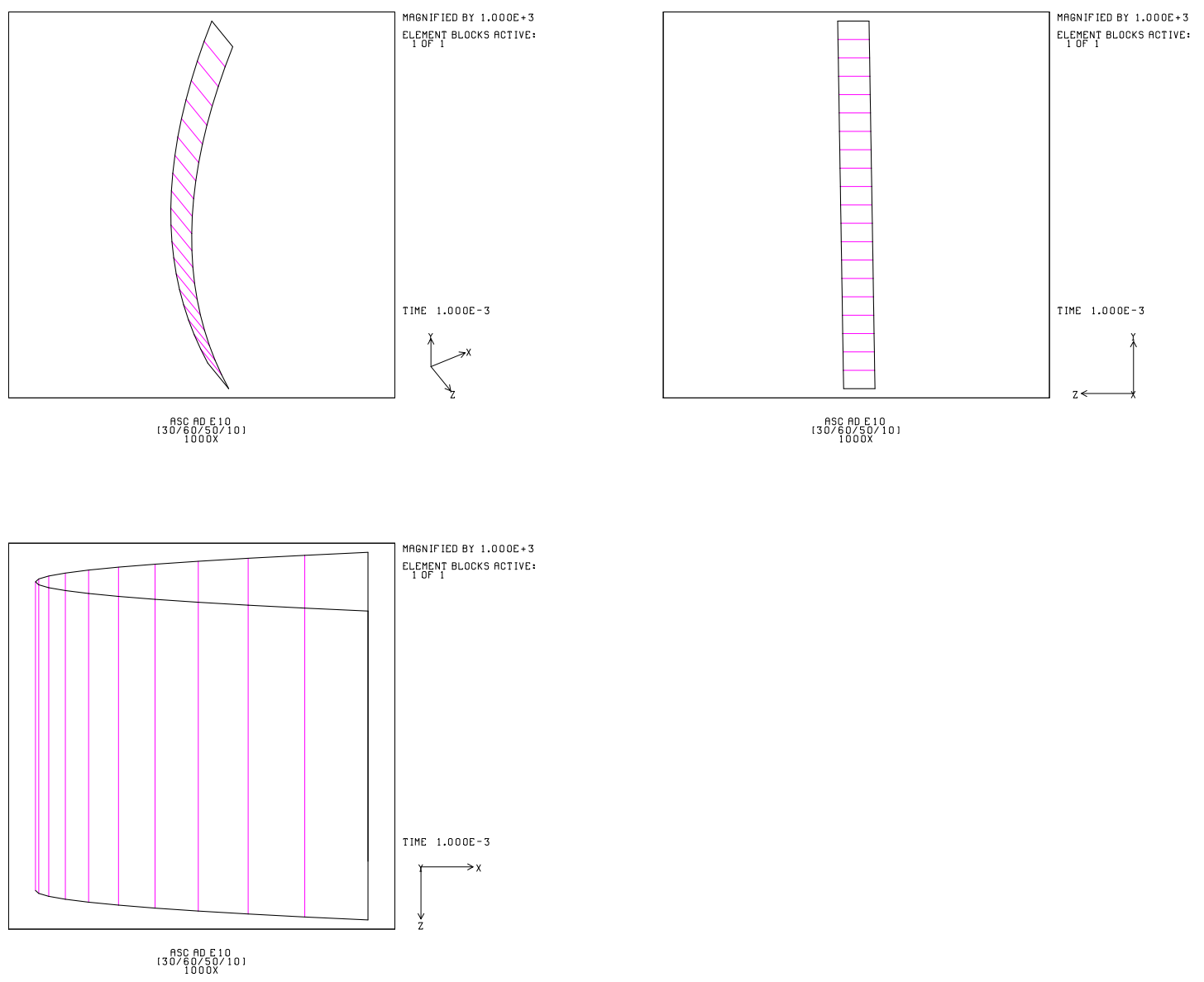

Figure 3.26: Deformed [30/60/50/10] Ring B shell model showing 1 element in circumferential direction. Deformations magnified by 1000 . 


\section{Composite Containers}

\subsection{Problem Definition}

In order to demonstrate the ability of ADAGIO to solve large problems involving composites, a composite container will be analyzed under internal pressure, thermal loads, and mechanical loading. The undeformed cylindrical container geometry is shown in Figure 4.1. Although a more realistic composite container would have portions made of metal, the container problem defined here is fully composed of a 4-ply laminated composite, except for the isotropic brackets near the symmetry plane at the center of the structure. Furthermore, a realistic container would also have rounded corners in the end caps instead of the sharp corners modeled here. Nevertheless, the chosen geometry is valid for demonstration purposes.

Each of the four layers in the composite has a thickness of $0.025 "(0.0635 \mathrm{~cm})$ for a total thickness of $0.1 "(0.254 \mathrm{~cm})$. Similar to the composite rings, the following material properties are chosen for each layer:

$$
\begin{aligned}
E_{11} & =64.03 \mathrm{GPa} \\
E_{22} & =55.50 \mathrm{GPa} \\
E_{33} & =9.78 \mathrm{GPa} \\
\nu_{12} & =0.081 \\
\nu_{23} & =0.303 \\
\nu_{31} & =0.0462805 \\
G_{12} & =G_{23}=G G_{31}=4.45 \mathrm{GPa} \\
\alpha_{11} & =\alpha_{22}=1 \mathrm{ppm} /{ }^{\circ} \mathrm{C} \\
\alpha_{33} & =30 \mathrm{ppm} /{ }^{\circ} \mathrm{C}
\end{aligned}
$$

The following stacking sequences are used: a symmetric cross-ply sequence of [0/90/90/0], a symmetric lay-up of [30/45/45/30], and an antisymmetric angle-ply arrangement of $[30 /-30 / 30 /-30]$. Here the first layer is the outermost layer of the structure, whereas the fourth layer is the innermost layer. In the cylindrical sidewalls, a $0^{\circ}$ fiber angle corresponds to the circumferential direction and a $90^{\circ}$ fiber angle corresponds to the axial direction. Likewise, for the end caps, the $0^{\circ}$ fiber angle 
$\mathrm{R}=7.908$ " $(20.086 \mathrm{~cm})$

$\mathrm{L}_{1}=1.768 "(4.491 \mathrm{~cm})$

$\mathrm{L}_{2}=4.175 "(10.605 \mathrm{~cm})$

$\mathrm{L}_{3}=1.0$ " $(2.54 \mathrm{~cm})$

$\mathrm{H}_{1}=15.513$ " $(39.403 \mathrm{~cm})$

$\mathrm{H}_{2}=1.175 "(2.985 \mathrm{~cm})$

$\mathrm{H}_{3}=1 "(2.54 \mathrm{~cm})$

$\mathrm{H}_{4}=2.04 "(5.182 \mathrm{~cm})$

$\mathrm{t}_{1}=0.1 "(0.254 \mathrm{~cm})$

$\mathrm{t}_{2}=0.2 "(0.508 \mathrm{~cm})$

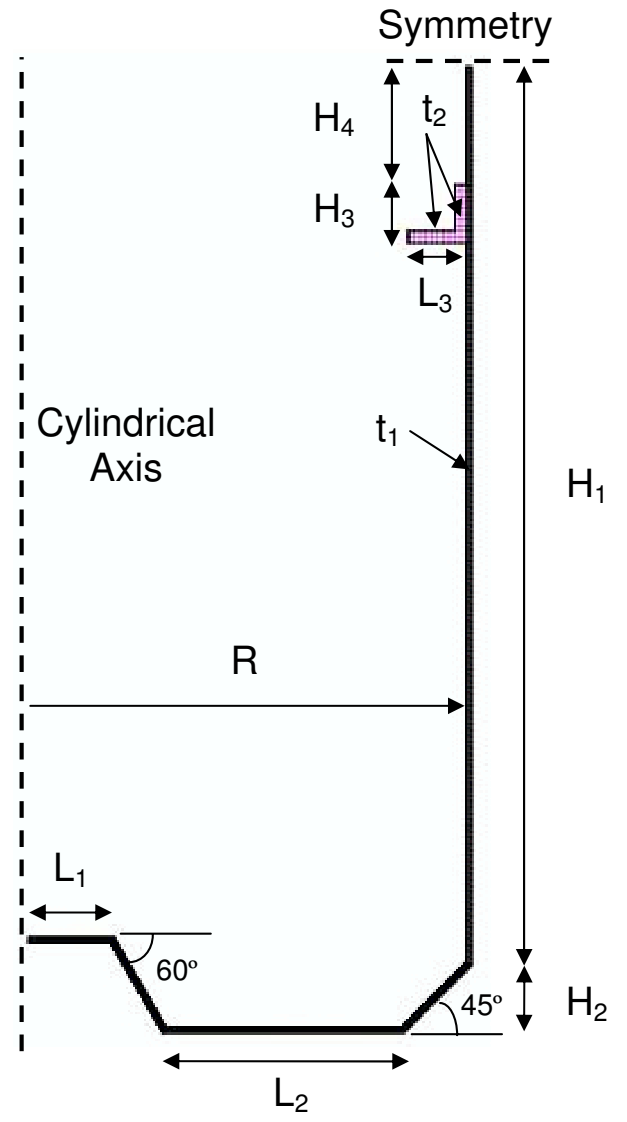

Figure 4.1: Composite container geometry. 
corresponds to the circumferential direction. For the horizontal portions of the end caps, a $90^{\circ}$ fiber angle gives fiber aligned in the radial direction. Finally, for the nonhorizontal portions of the end caps (the $-60^{\circ}$ and $45^{\circ}$ segments), a $90^{\circ}$ fiber direction is perpendicular to the circumferential direction, but parallel to the local container surface. Once again, other fiber angles can be determined by a simple rotation in the plane defined by the $0^{\circ}$ and $90^{\circ}$ fiber directions. The isotropic brackets to which the mechanical load will be applied are taken to have a Youngs modulus of $64.03 \mathrm{GPa}$, a shear modulus of 24.6 GPa, and a coefficient of thermal expansion of $11.7 \mathrm{ppm} / \mathrm{C}$.

Finally, in order to produce a usable mesh using SEACAS based tools, a small hole is drilled in the center of the end cap. This hole is small and no radial displacements are applied to its edge such that it is equivalent to having a small plug of infinite stiffness in the radial direction. Although other meshing schemes could have been used where this step would have not been required, those schemes are not as straightforward as the one used here where a $2 \mathrm{D}$ cross-section is used to sweep out the full 3D geometry.

Both continuum and combined shell/continuum approaches will be used to analyze the composite container response. Because one purpose of this project is to demonstrate the solution of composite problems using parallel processing, the entire $360^{\circ}$ structure is modeled, as periodic boundary conditions were not parallel aware in ADAGIO at the time these of analyses. For the purely continuum models, a mesh with a total of 467,520 elements and 585,840 nodes is used. With each continuum node having 3 degrees-of-freedom (DOF), the continuum model possesses a total of $1,757,520$ DOF before the application of boundary conditions. The combined shell/continuum approach consists of modeling all of the composite parts (everything but the brackets) with shell elements and using continuum elements for the brackets. The combined continuum/shell approach uses a mesh with 123,360 elements and 126,000 nodes. With each shell node having 6 DOF (three translations and three rotations), a total of 722,742 DOF are used in the combined shell/continuum approach, not counting the reduction in DOF from the application of boundary conditions. Note that when the 4-layer composite is modeled using continuum elements, it is necessary to use a minimum of 4 elements through the thickness (the approach chosen here) with orthotropic properties input for each layer, whereas for the shell modeling, a single element can be used to represent the entire lay-up with the laminate matrices input directly. Although 
using 1 element per layer is the minimum requirement for modeling composites using continuum elements, it may be necessary to use more elements to adequately capture all aspects of the composite response, such as bending effects in and around various geometric features.

\subsection{Response of Composite Containers to Pressure, Thermal, and Mechanical Loadings}

The composite containers are subjected to an internal pressurization of 1 psi (6894 $\mathrm{Pa})$, followed by a $18{ }^{\circ} \mathrm{F}\left(10{ }^{\circ} \mathrm{C}\right)$ temperature change, and finally a $0.027 \mathrm{psi}(187.69$ $\mathrm{Pa}$ ) distributed loading on the horizontal surface of the load bracket shown in Figure 4.1. The distributed loading of $0.027 \mathrm{psi}$ corresponds to a $1 \mathrm{lb}$ force subjected over the $36.734 \mathrm{in}^{2}$ horizontal surface area of the load bracket. Recall that symmetry boundary conditions are used at the top of the geometry shown in Figure 4.1 such that the final results include the effects of loading on a lower bracket (shown) and an upper bracket (not shown). Each of the three types of loads are ramped-up linearly over a specified time range and then held constant for the rest of the analysis. The internal pressurization increases from zero to its final value over the first $0.001 \mathrm{sec}$, the thermal loading is applied from 0.0001 to $0.0005 \mathrm{sec}$, and finally the distributed mechanical loading of the bracket is ramped-up over $0.0005 \mathrm{sec}$ to $0.001 \mathrm{sec}$. A total of 1000 time steps are used for applying the internal pressurization, while 500 time steps are used for applying both the thermal and mechanical loadings for a total of 2000 time steps. It is unknown how close to optimality such a time stepping scenario is to one requiring the least $\mathrm{CPU}$ usage. Note that the time variable here is used as the means to control the loadings and that the composite response at any given load level is time independent, as only linear elastic models are being employed.

The parallel finite element runs were performed using Sandia's Rogue Linux cluster. A summary of the number of processors used and the total CPU time expended for each analysis is provided in Table 4.1. Most jobs were run in the slower Serial queue of Rogue. The shell analyses were attempted using the probe nodal preconditioner. For this preconditioner, only the [0/90/90/0] container problem was able to be converged. Additional preliminary attempts to simulate the $[30 / 45 / 45 / 30]$ and $[30 /-$ 30/30/-30] containers using the full tangent preconditioner with FETI linear solver 
Table 4.1: CPU usage for composite container analyses.

\begin{tabular}{|l|l|l|l|l|l|l|l|}
\hline $\begin{array}{l}\text { Stacking } \\
\text { Sequence }\end{array}$ & Model $^{+}$ & Elements & Nodes & DOF & $\begin{array}{l}\text { Rogue } \\
\text { Queue }\end{array}$ & $\begin{array}{l}\text { No. } \\
\text { of } \\
\text { CPU }\end{array}$ & $\begin{array}{l}\text { Total } \\
\text { CPU } \\
\text { Time } \\
\text { (hr })\end{array}$ \\
\hline$[0 / 90 / 90 / 0]$ & $\mathrm{C}$ & 467,520 & 585,840 & $1,757,520$ & Serial & 36 & 2552 \\
\hline$[30 / 45 / 45 / 30]$ & $\mathrm{C}$ & 467,520 & 585,840 & $1,757,520$ & Scico & 36 & $3460^{*}$ \\
\hline$[30 /-30 / 30 /-30]$ & $\mathrm{C}$ & 467,520 & 585,840 & $1,757,520$ & Serial & 36 & 2700 \\
\hline$[0 / 90 / 90 / 0]$ & & & & & & & \\
\hline$[30 / 45 / 45 / 30]$ & $\mathrm{S} / \mathrm{C}$ & 123,360 & 126,000 & 722,742 & Serial & 36 & 1560 \\
\hline$[30 /-30 / 30 /-30]$ & $\mathrm{S} / \mathrm{C}$ & 123,360 & 126,000 & 722,742 & - & - & - \\
\hline
\end{tabular}

${ }^{+} \mathrm{C}=$ continuum, $\mathrm{S}=$ shell,

$\mathrm{S} / \mathrm{C}=$ shell for all composite walls, continuum for load bracket

*Estimated (some data lost due to restart)

were unsuccessful.

Figures 4.2-4.5 show the deformed cross-sections. Note that the deformations in those figures have been multiplied by ten to aid viewing. Similar deformations result in each case. It can be observed that much of the displacements in the end cap result from bending response at the intersection of the various structural segments. Figures 4.6-4.8 show contour plots of the von Mises stresses for the purely continuum models on a layer-by-layer basis. These von Mises stress distributions are comparable for the stacking sequences considered. However, it is likely that the distribution in the actual tensor components of the stress differ for each stacking sequence.

Figures 4.9-4.14 show the layerwise stress results due only to internal pressurization for the $[0 / 90 / 90 / 0]$ container modeled using solely continuum elements, whereas Figures 4.15-4.20 give the corresponding layerwise stress results when shell elements are used for everything except the load bracket. Note that the load bracket is not shown in any of these figures for convenience. The agreement in the minimum and maximum stresses between the two approaches are better for the outermost and in- 


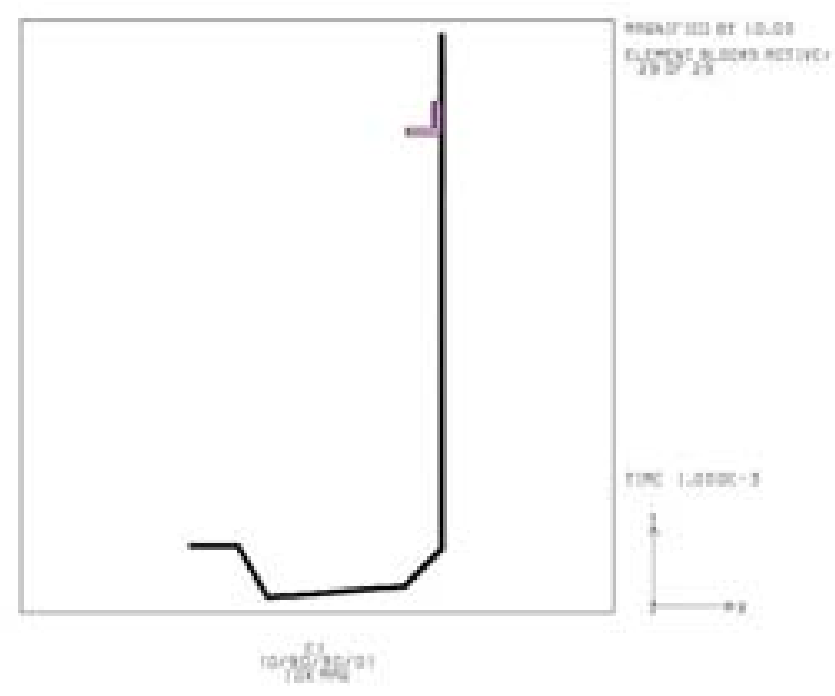

Figure 4.2: Cross sectional view of composite container with [0/90/90/0] lay-up modeled using only continuum elements with 10X mag. of final displacements.

nermost layers than for the inner two layers. These peaks and valleys in the stress distributions occur at the corners between the various container segments where there is significant bending. Hence, one possible explanation for the better agreement in stresses on the outer and inner layers is that bending effects dominate in these regions, the outermost and innermost layers naturally have higher bending strains than the inner two layers, and the shell model is able to capture these bending effects fairly well. A complete explanation requires additional study which was not possible under the time constraints of this project. 


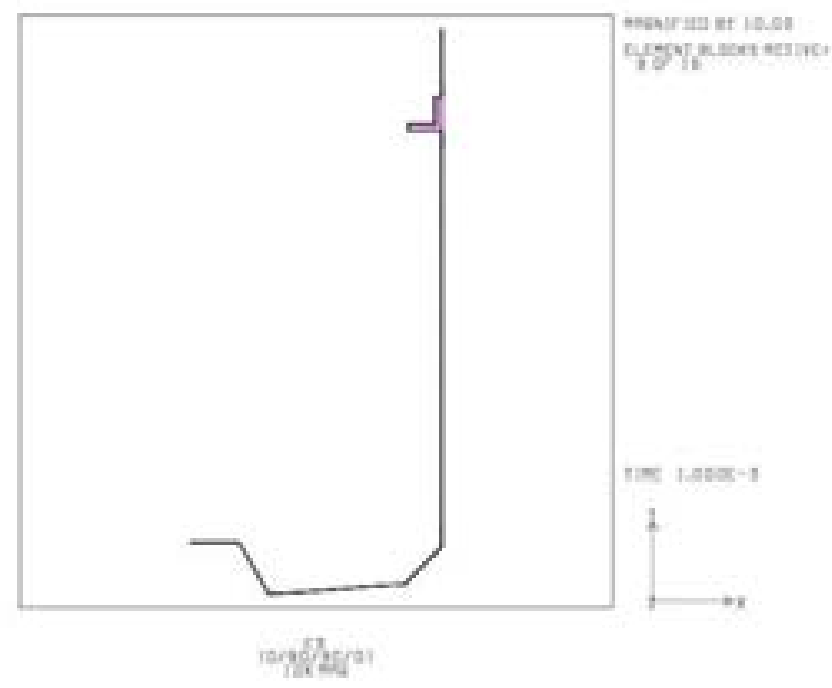

Figure 4.3: Cross sectional view of composite container with [0/90/90/0] lay-up modeled using only primarily shell elements with 10X mag. of final displacements.

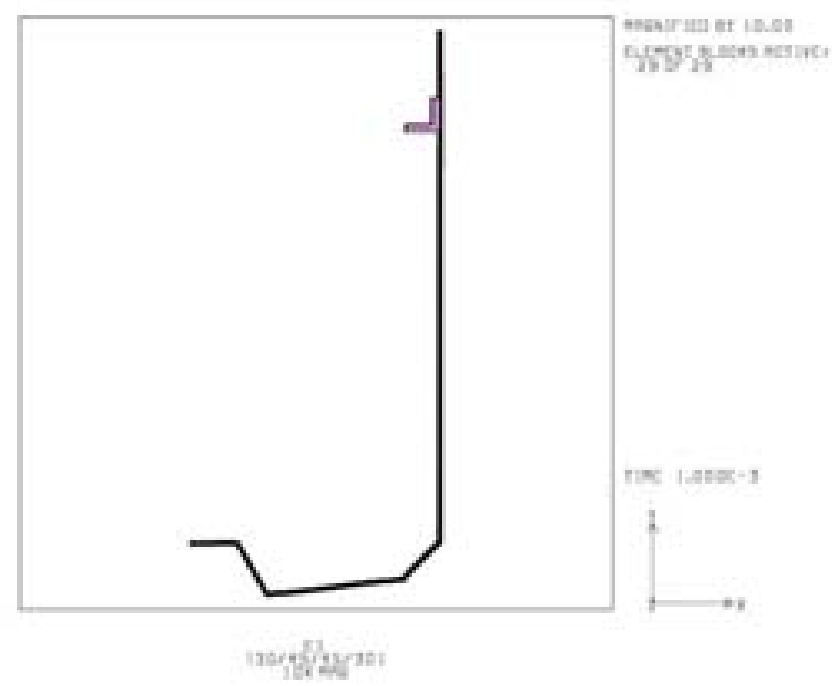

Figure 4.4: Cross sectional view of composite container with [30/45/45/30] lay-up modeled using only continuum elements with 10X mag. of final displacements. 


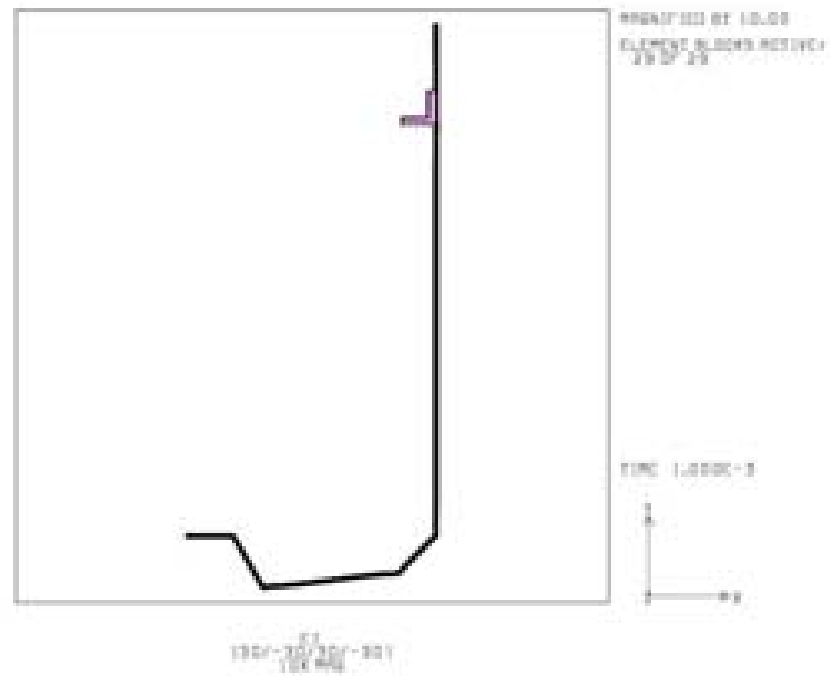

Figure 4.5: Cross sectional view of composite container with [30/-30/30/-30] lay-up modeled using only continuum elements with 10X mag. of final displacements. 
(a)

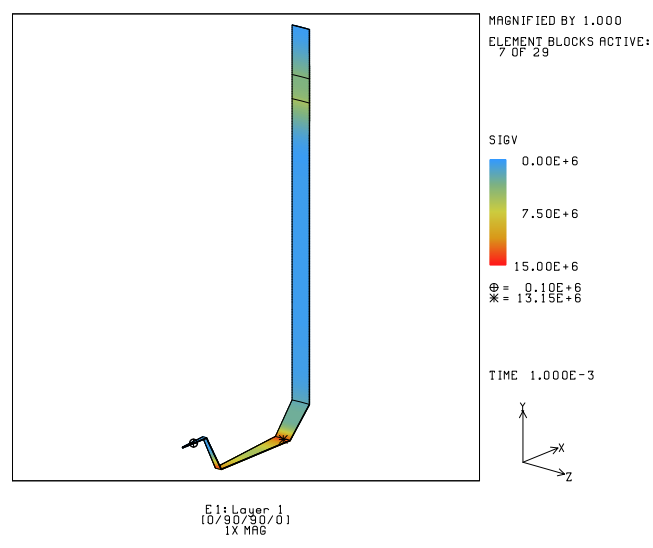

(c)

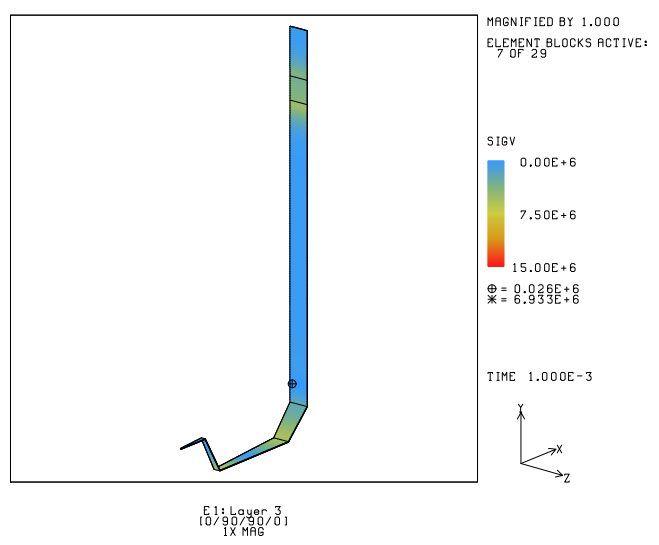

(b)

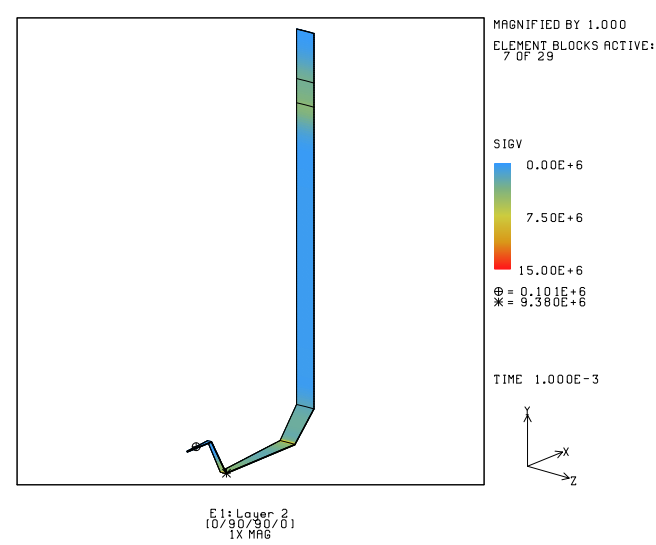

(d)

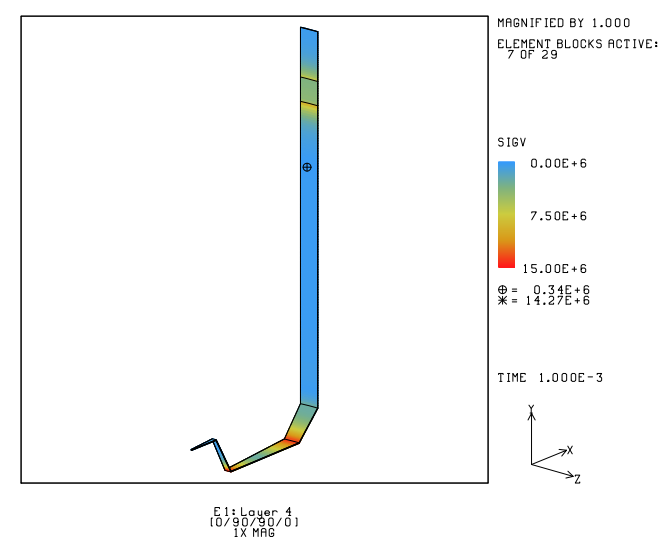

Figure 4.6: von Mises stresses due to internal pressurization, thermal loading, and mechanical loading for the $[0 / 90 / 90 / 0]$ container modeled using only continuum elements: (a) layer 1 (outermost layer); (b) layer 2; (c) layer 3; (d) layer 4 (innermost layer). 
(a)

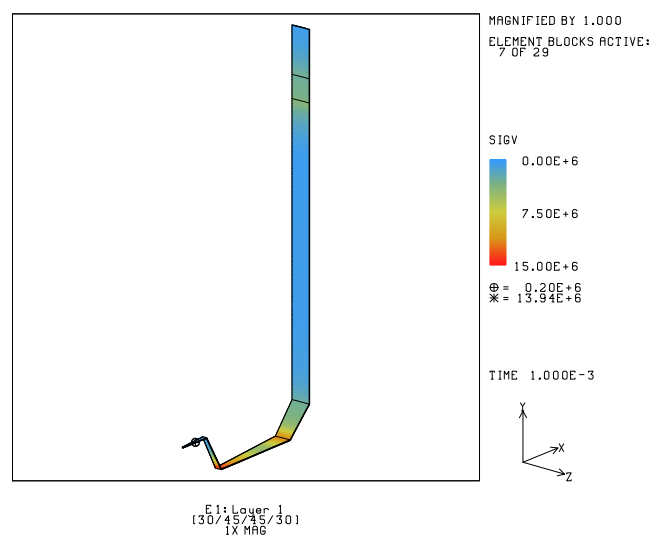

(c)

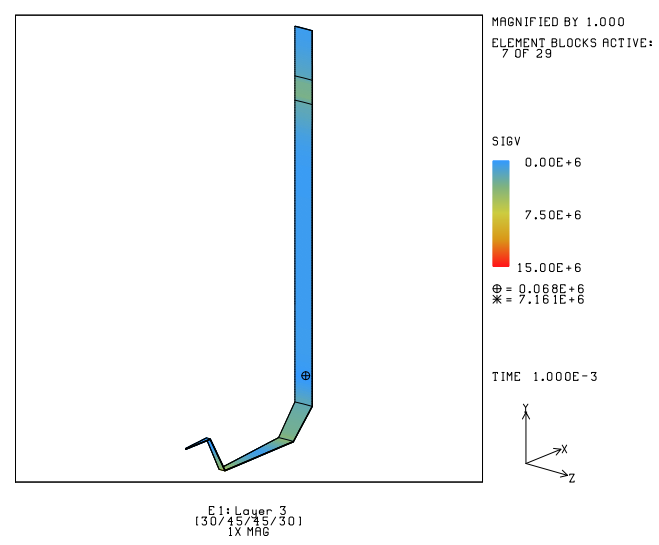

(b)

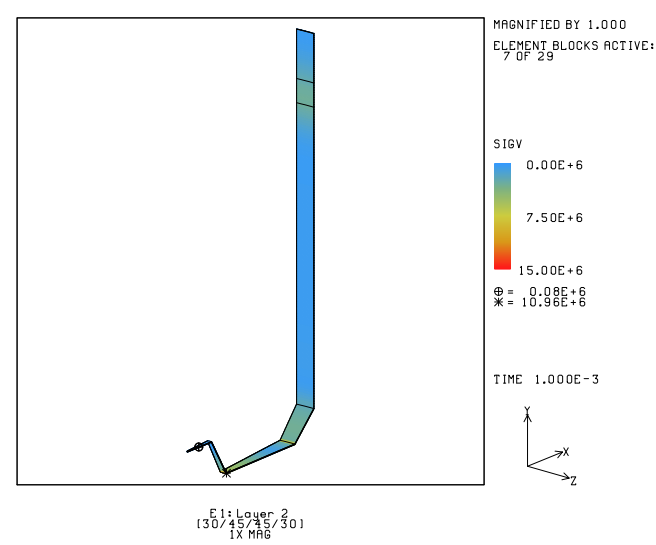

(d)

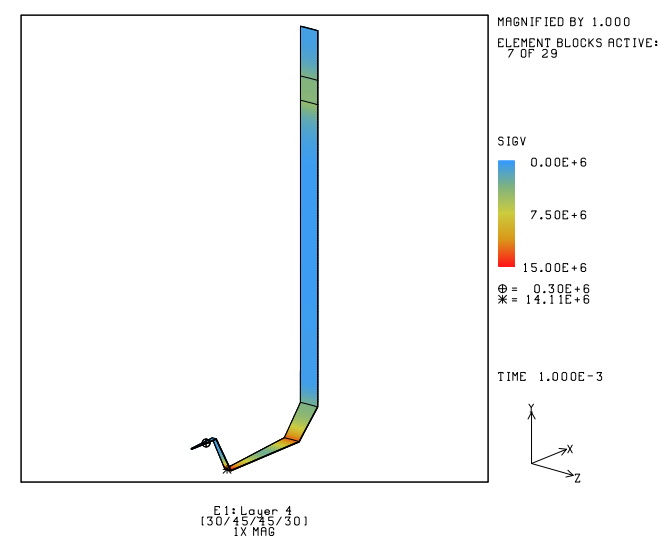

Figure 4.7: von Mises stresses due to internal pressurization, thermal loading, and mechanical loading for the $[30 / 45 / 45 / 30]$ container modeled using only continuum elements: (a) layer 1 (outermost layer); (b) layer 2; (c) layer 3; (d) layer 4 (innermost layer). 
(a)

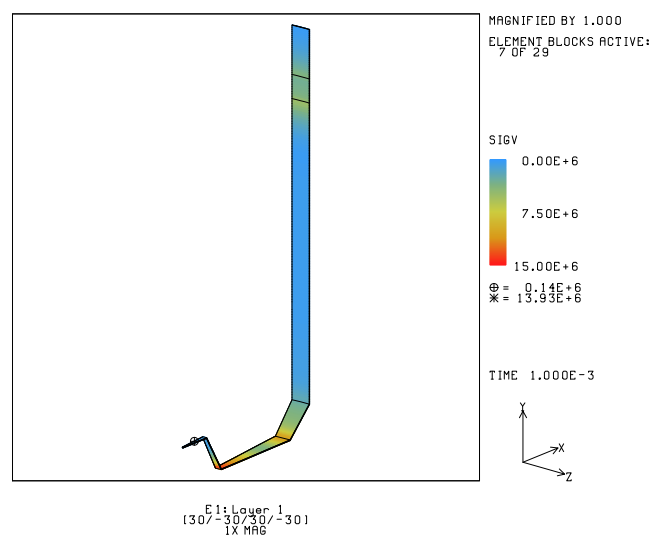

(c)

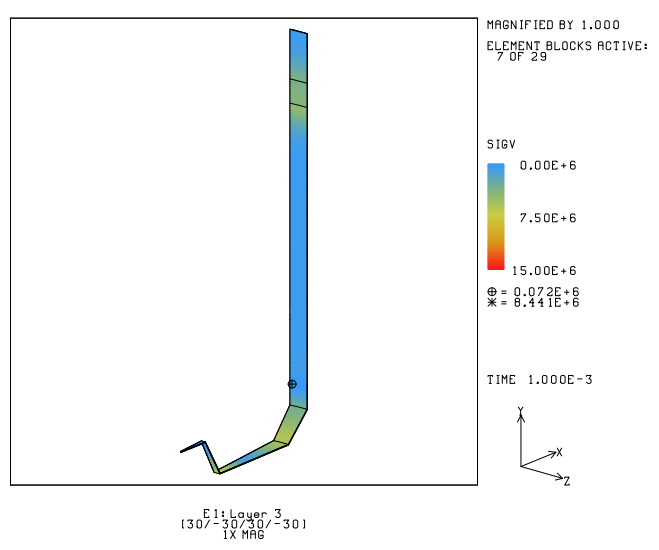

(b)

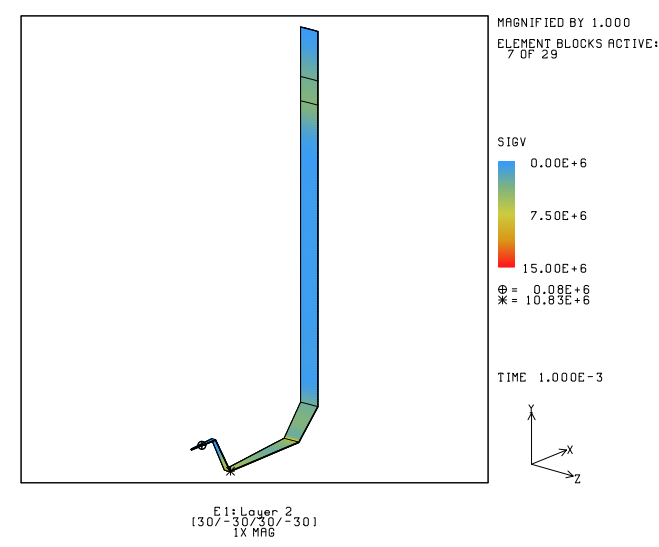

(d)

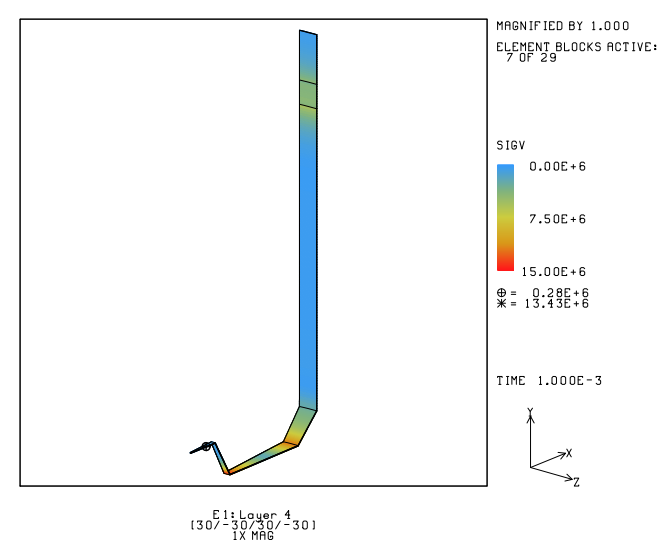

Figure 4.8: von Mises stresses due to internal pressurization, thermal loading, and mechanical loading for the [30/-30/30/-30] container modeled using only continuum elements: (a) layer 1 (outermost layer); (b) layer 2; (c) layer 3; (d) layer 4 (innermost layer). 
(a)

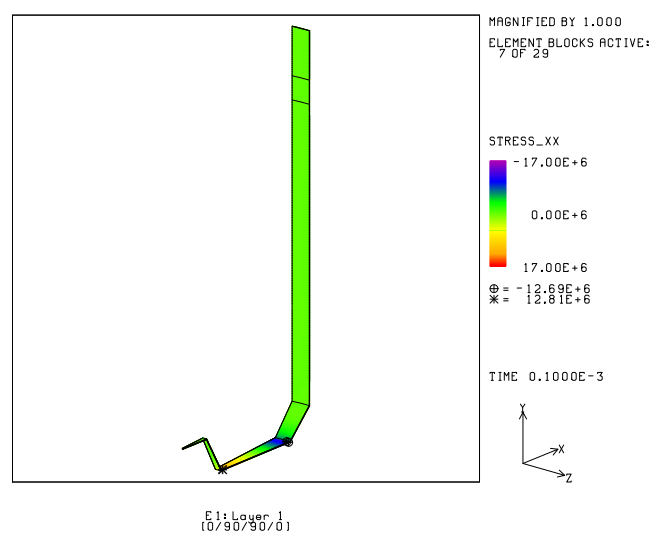

(c)

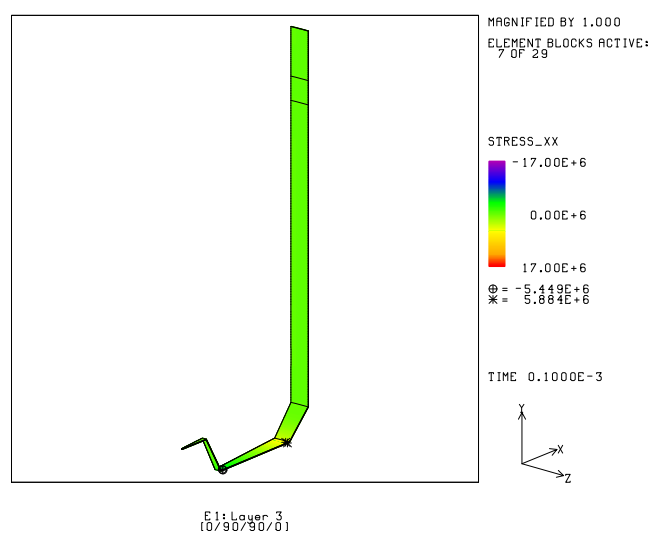

(b)

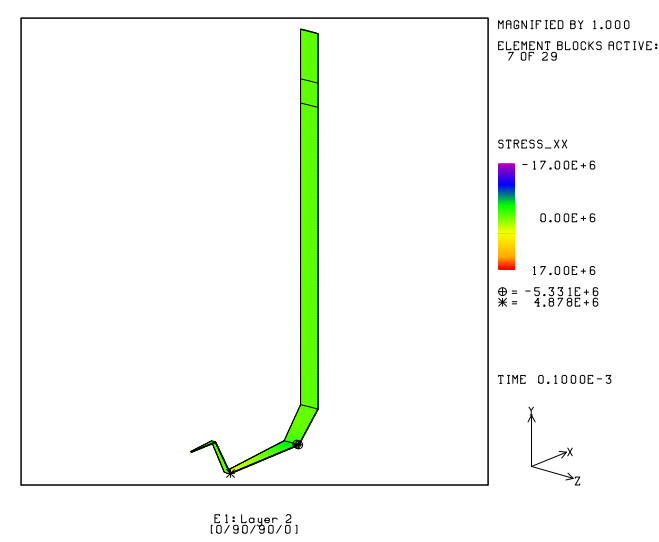

(d)

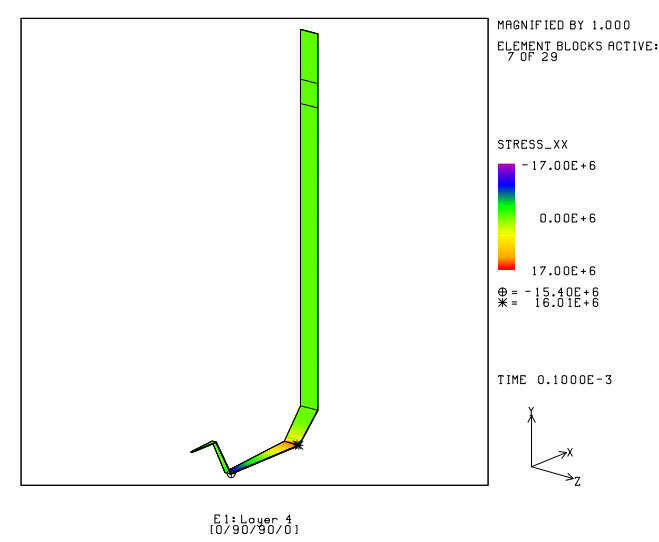

Figure 4.9: Radial stresses due to internal pressurization for the [0/90/90/0] container modeled using only continuum elements: (a) layer 1 (outermost layer); (b) layer 2; (c) layer 3; (d) layer 4 (innermost layer). 
(a)

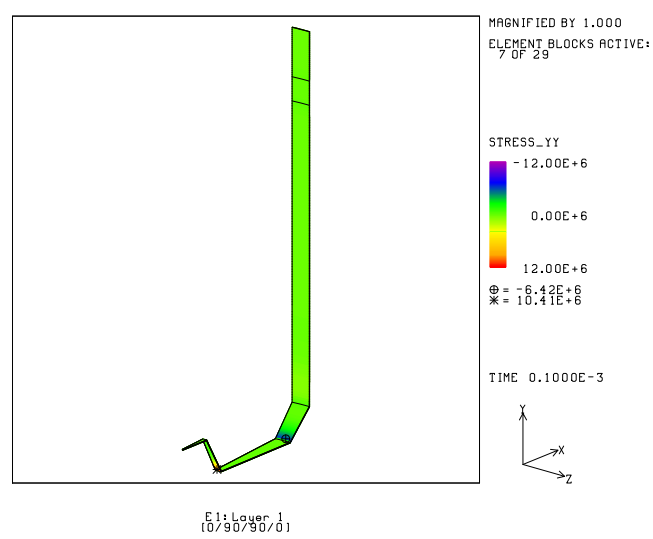

(c)

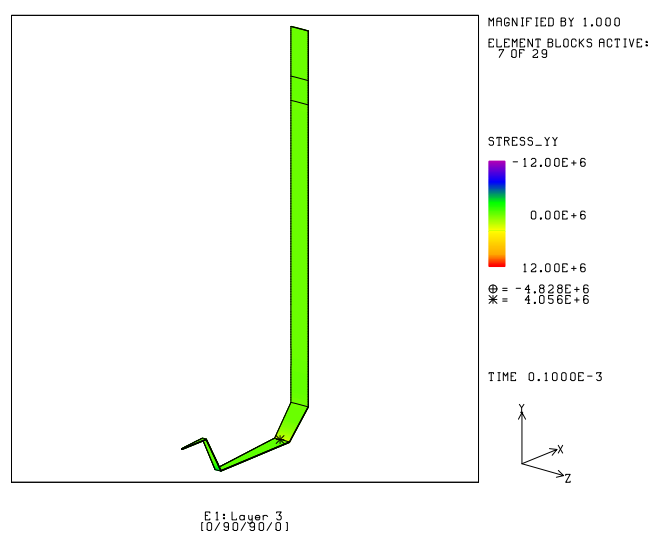

(b)

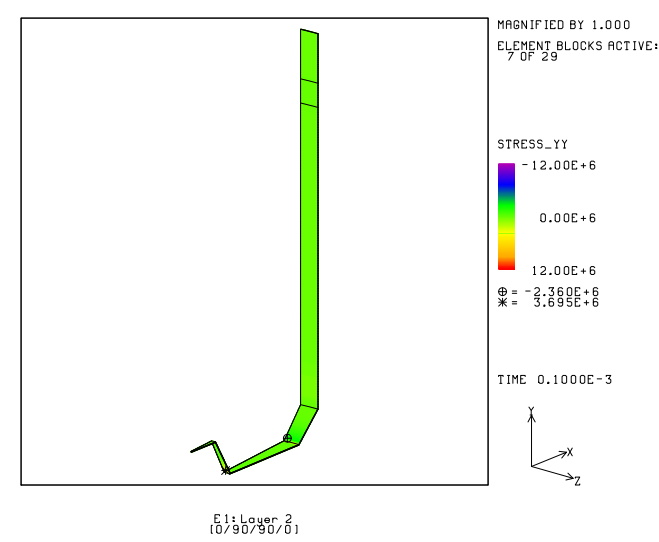

(d)

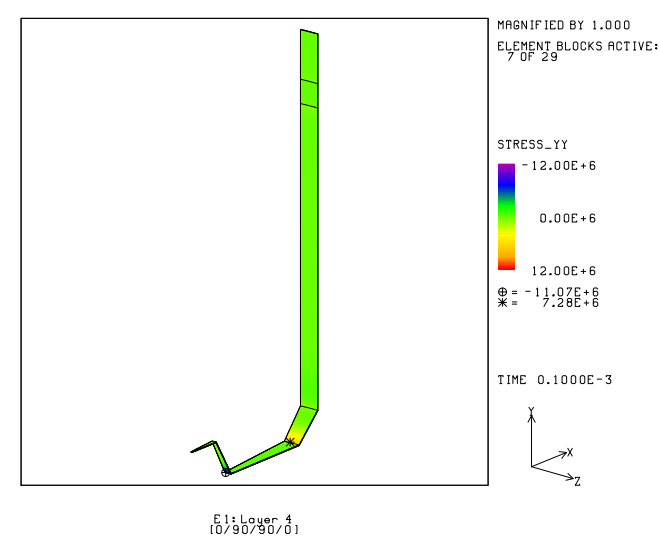

Figure 4.10: Axial stresses due to internal pressurization for the [0/90/90/0] container modeled using only continuum elements: (a) layer 1 (outermost layer); (b) layer 2; (c) layer 3; (d) layer 4 (innermost layer). 
(a)

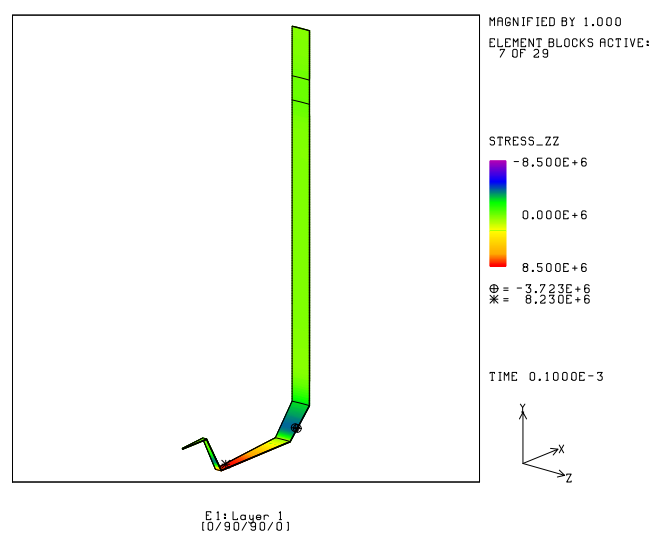

(c)

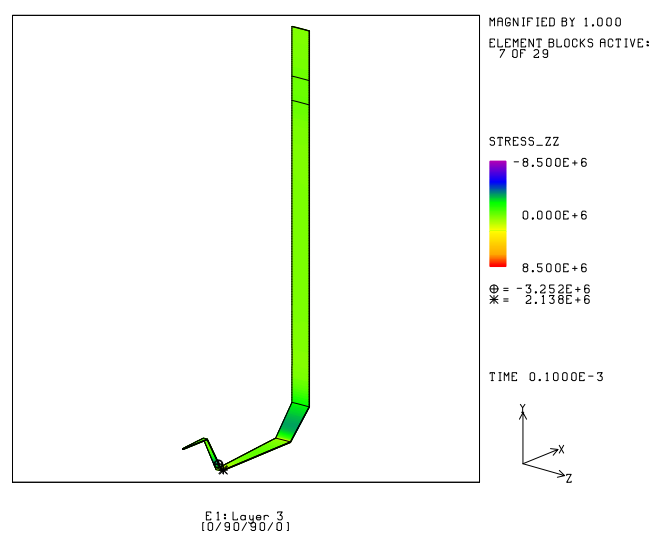

(b)

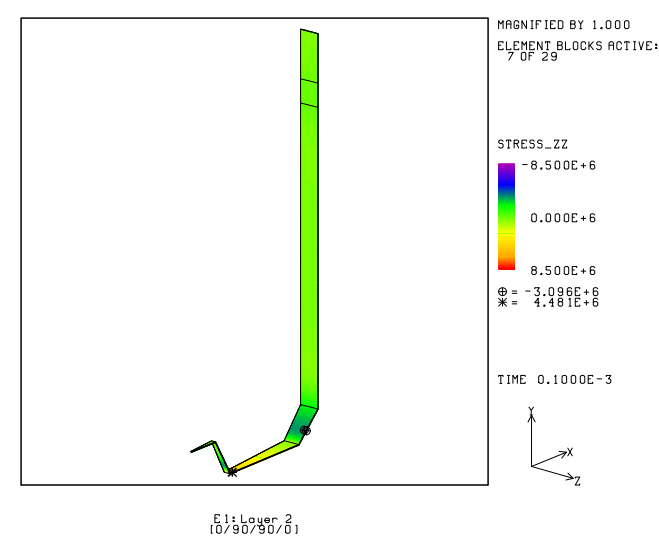

(d)

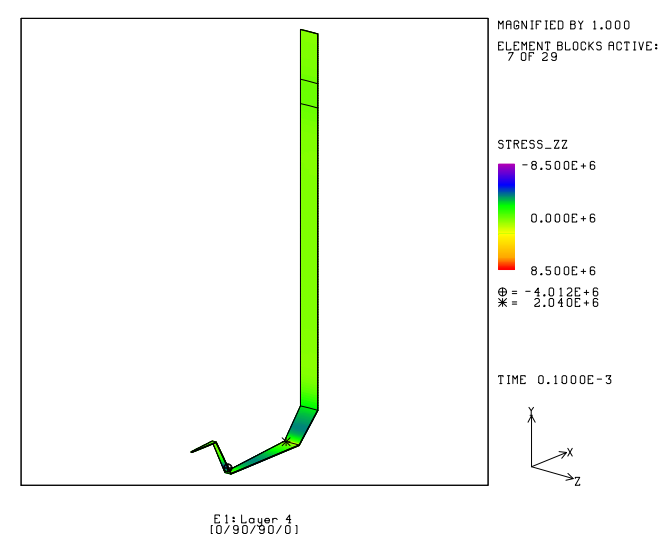

Figure 4.11: Circumferential stresses due to internal pressurization for the [0/90/90/0] container modeled using only continuum elements: (a) layer 1 (outermost layer); (b) layer 2; (c) layer 3; (d) layer 4 (innermost layer). 
(a)

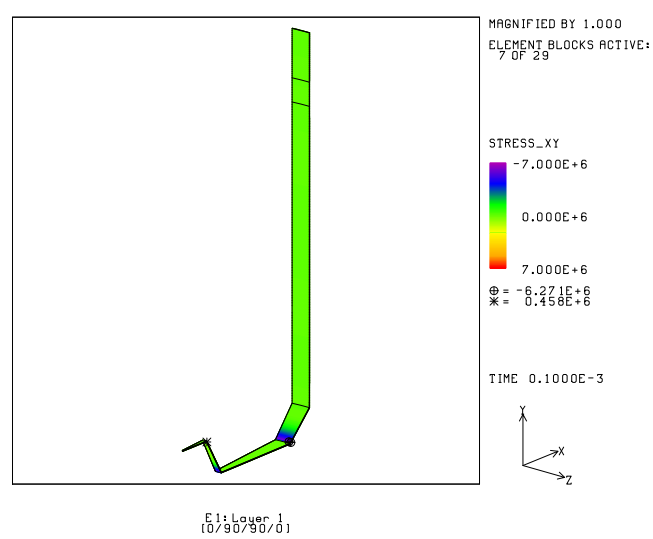

(c)

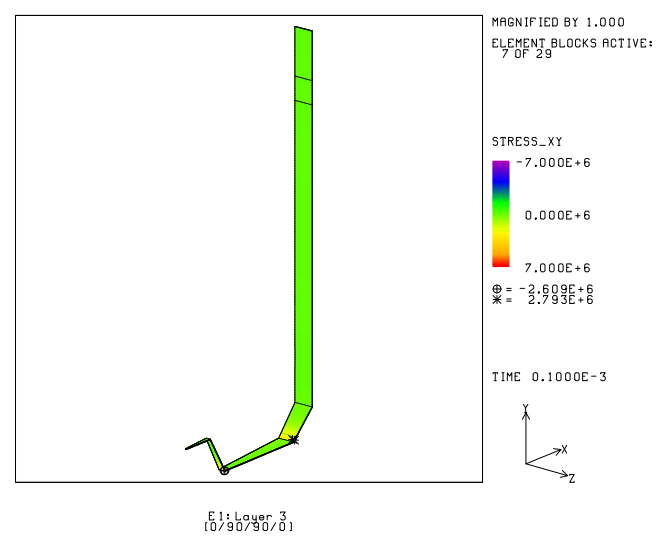

(b)

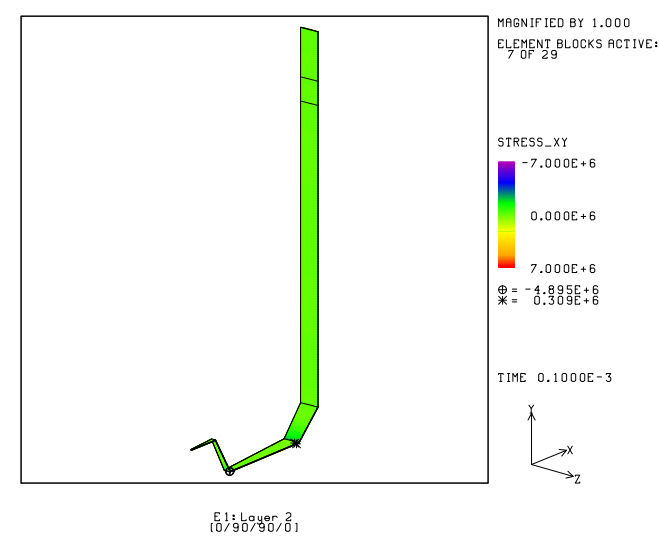

(d)

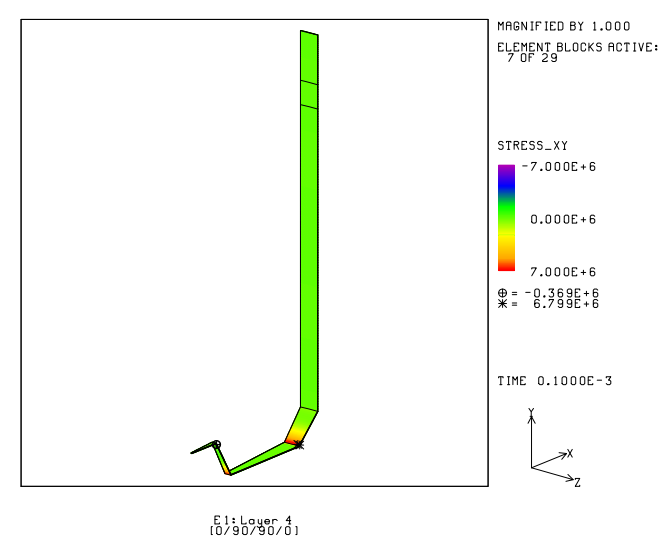

Figure 4.12: Radial-axial shear stresses due to internal pressurization for the [0/90/90/0] container modeled using only continuum elements: (a) layer 1 (outermost layer); (b) layer 2; (c) layer 3; (d) layer 4 (innermost layer). 
(a)

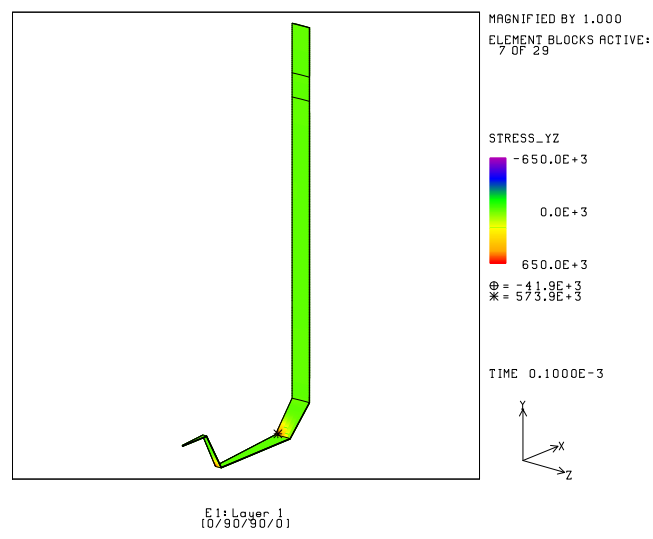

(c)

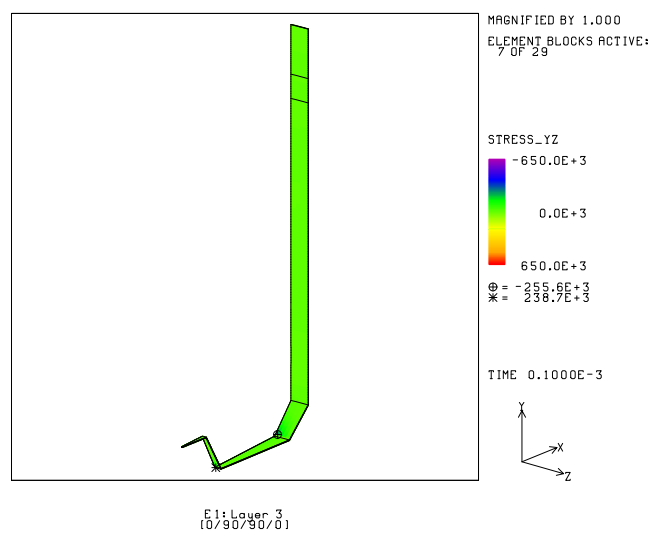

(b)

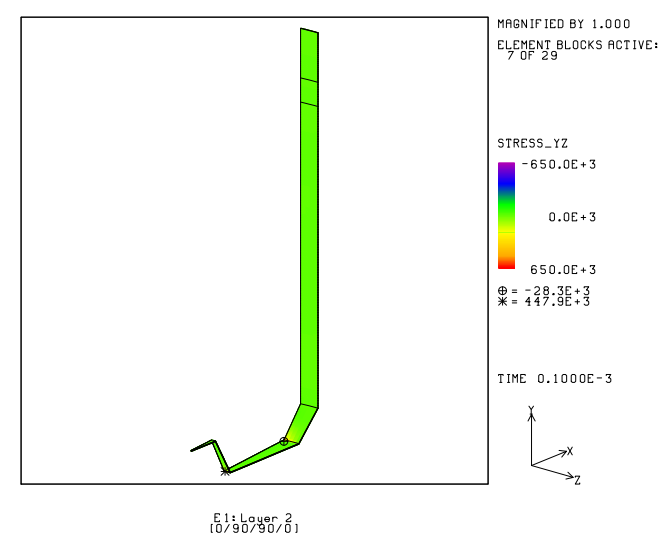

(d)

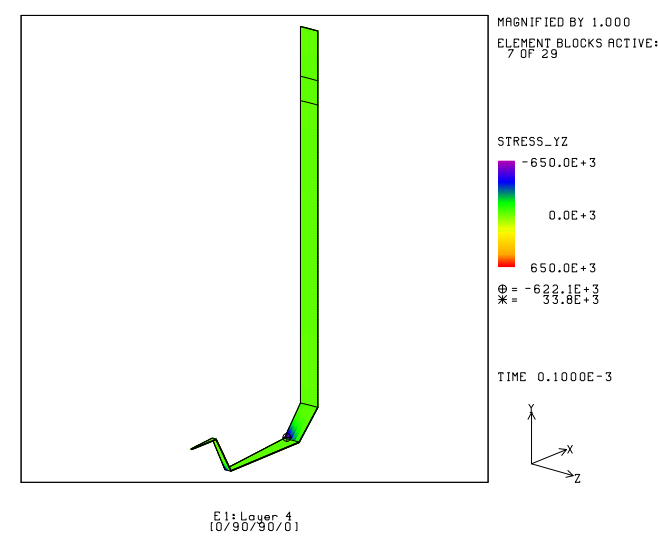

Figure 4.13: Negative of axial-circumferential shear stresses due to internal pressurization for the [0/90/90/0] container modeled using only continuum elements: (a) layer 1 (outermost layer); (b) layer 2; (c) layer 3; (d) layer 4 (innermost layer). 
(a)

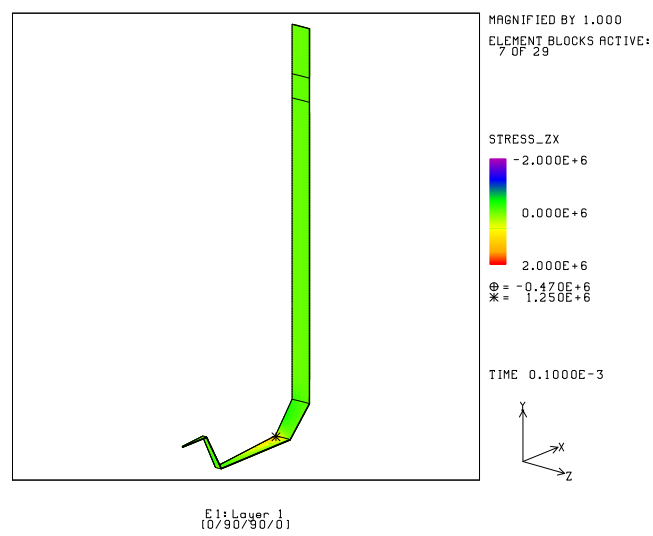

(c)

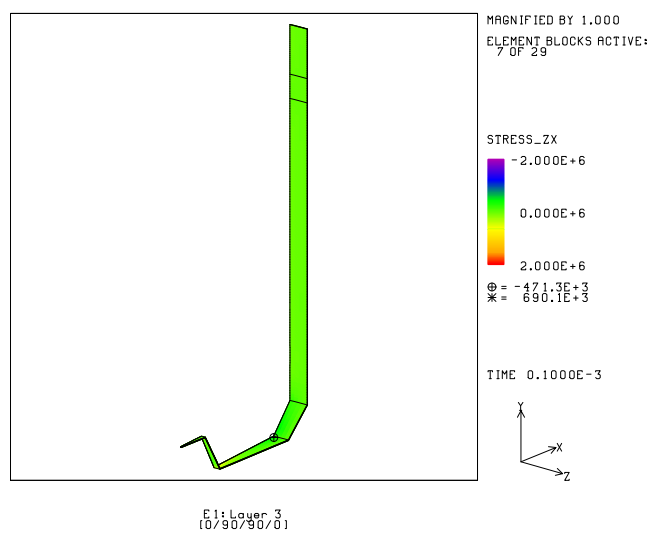

(b)

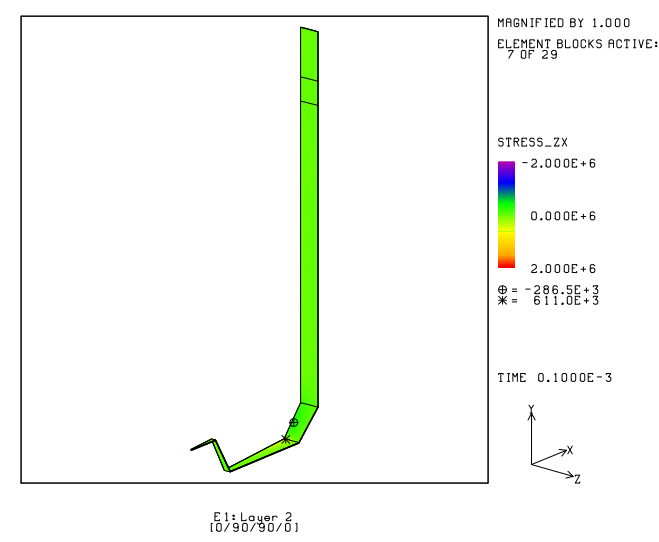

(d)

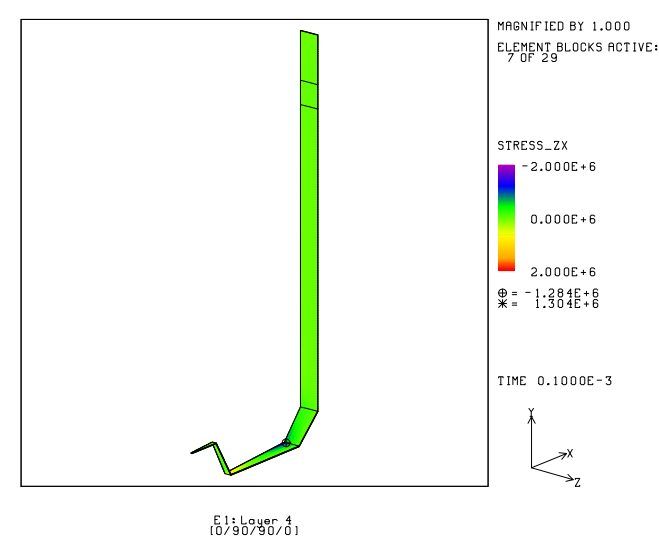

Figure 4.14: Negative of circumferential-radial shear stresses due to internal pressurization for the $[0 / 90 / 90 / 0]$ container modeled using only continuum elements: (a) layer 1 (outermost layer); (b) layer 2; (c) layer 3; (d) layer 4 (innermost layer). 
(a)

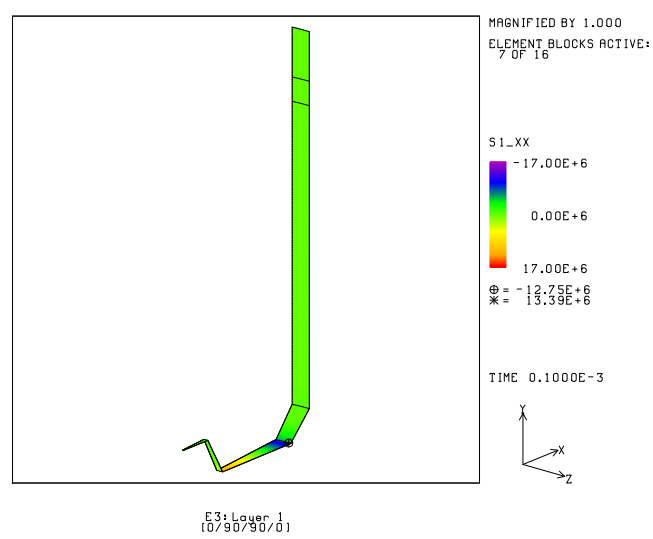

(c)

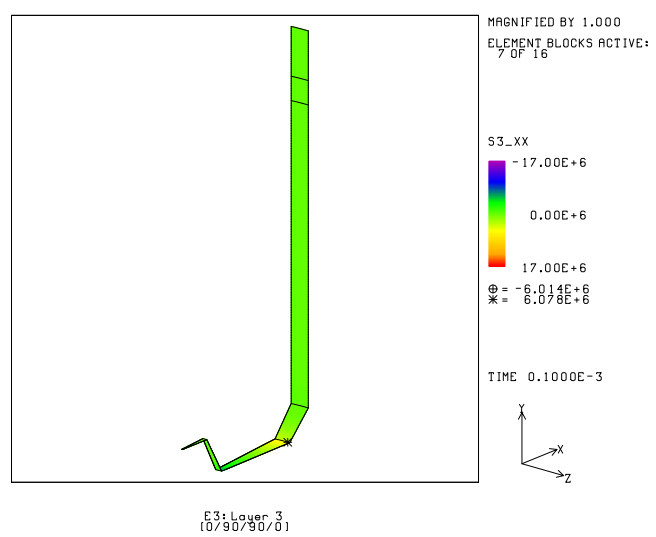

(b)

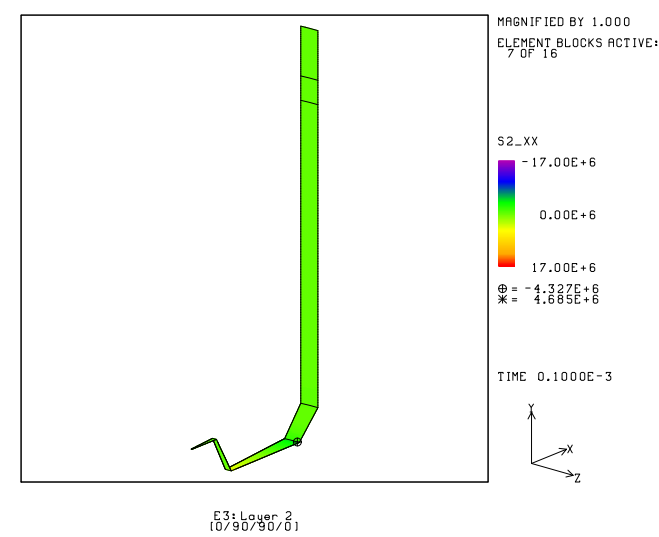

(d)

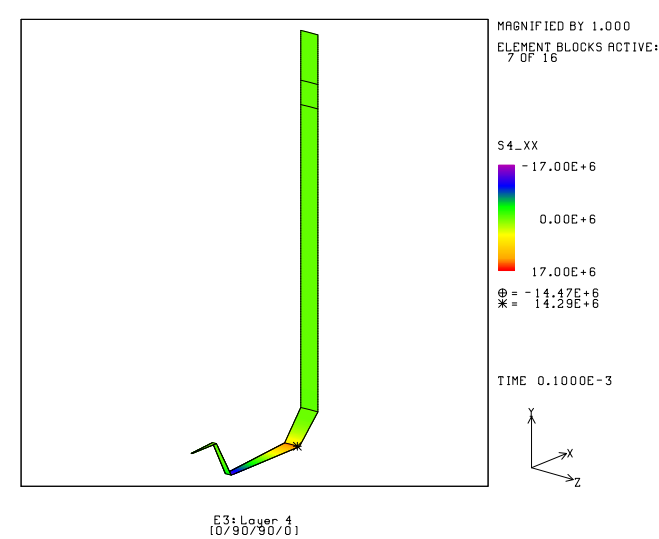

Figure 4.15: Radial stresses due to internal pressurization for the $[0 / 90 / 90 / 0]$ container modeled using primarily shell elements: (a) layer 1 (outermost layer); (b) layer 2; (c) layer 3; (d) layer 4 (innermost layer). 
(a)

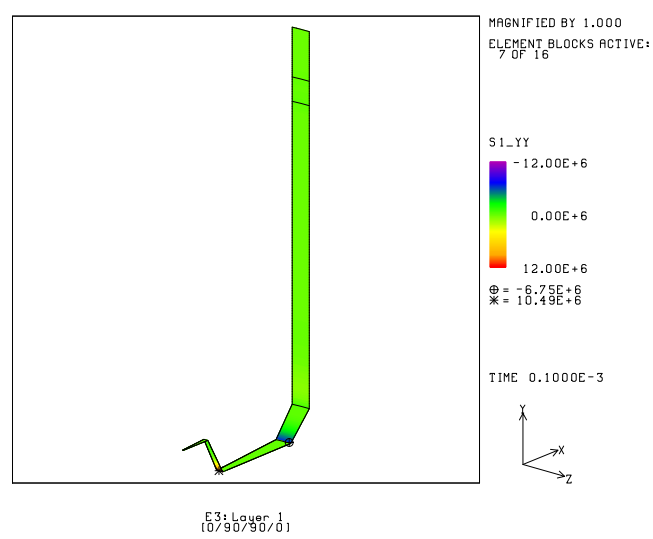

(c)

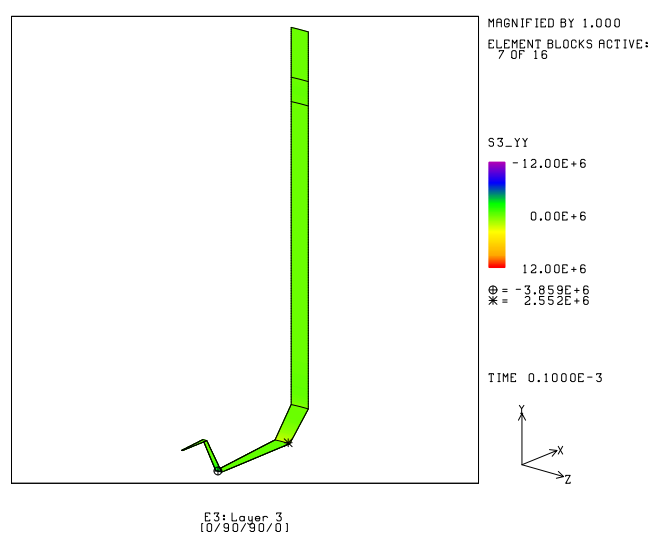

(b)

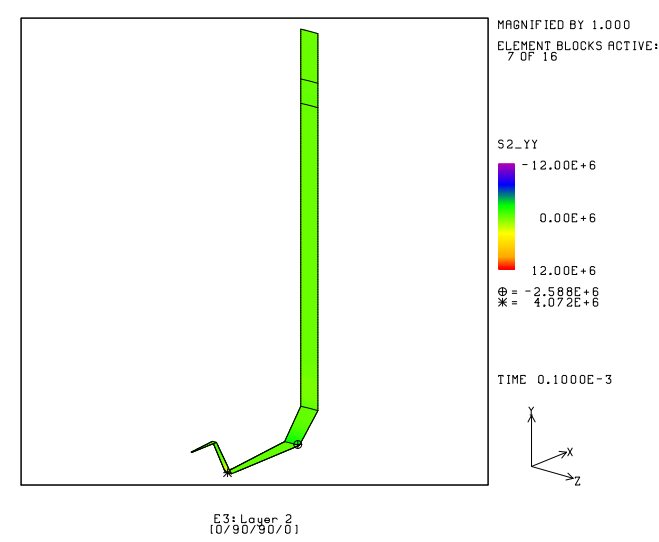

(d)

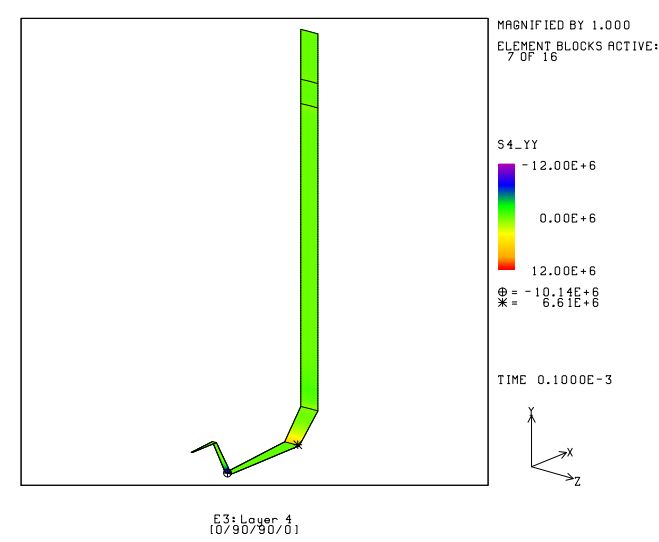

Figure 4.16: Axial stresses due to internal pressurization for the [0/90/90/0] container modeled using primarily shell elements: (a) layer 1 (outermost layer); (b) layer 2; (c) layer 3; (d) layer 4 (innermost layer). 
(a)

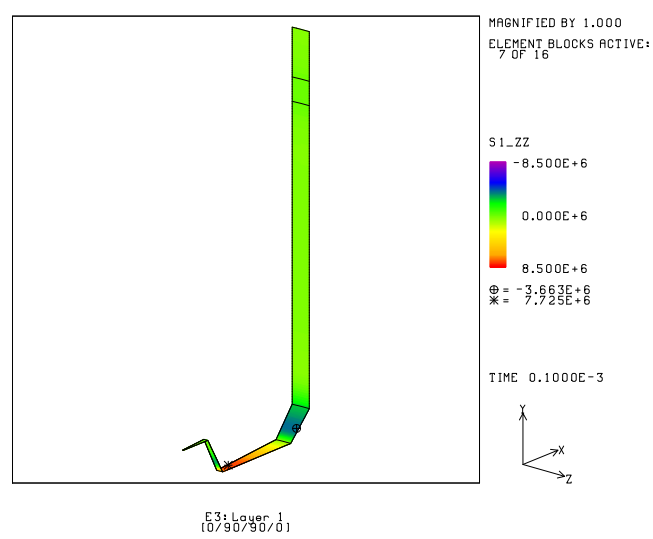

(c)

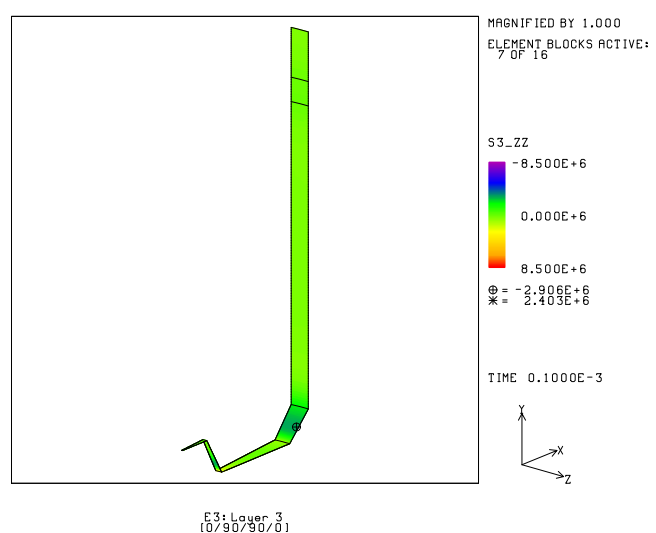

(b)

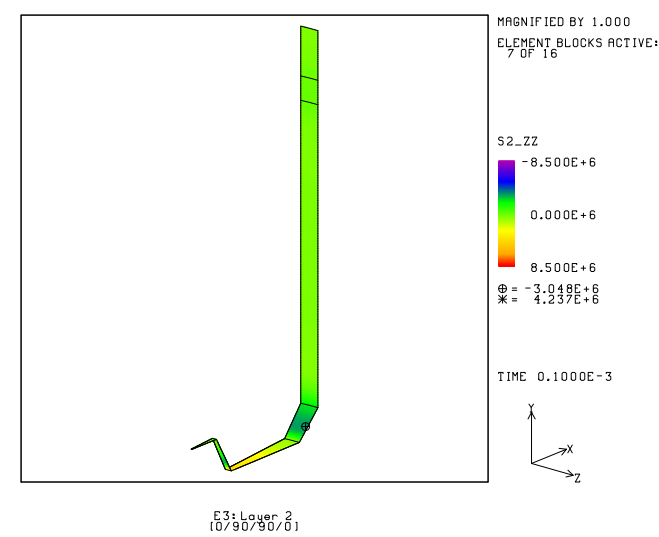

(d)

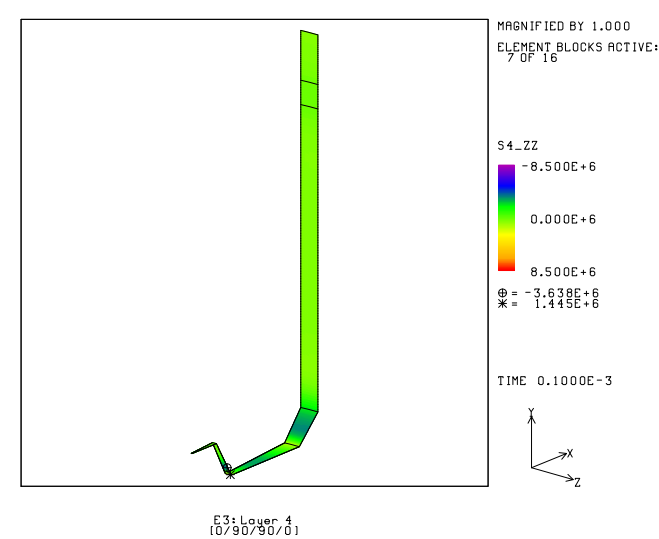

Figure 4.17: Circumferential stresses due to internal pressurization for the [0/90/90/0] container modeled using primarily shell elements: (a) layer 1 (outermost layer); (b) layer 2; (c) layer 3; (d) layer 4 (innermost layer). 
(a)

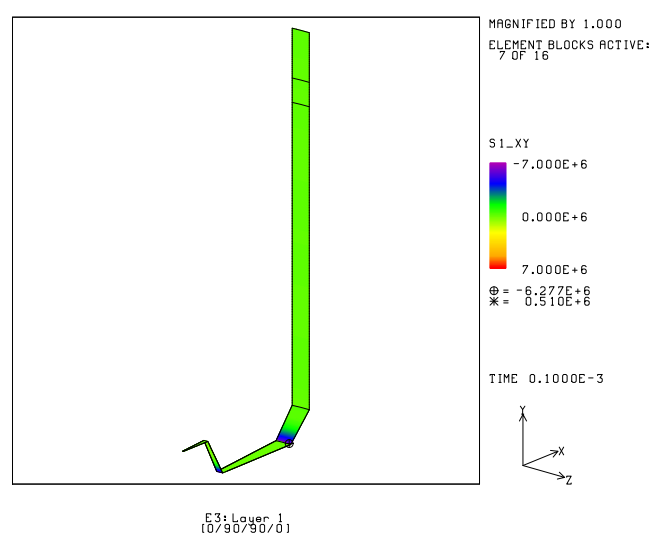

(c)

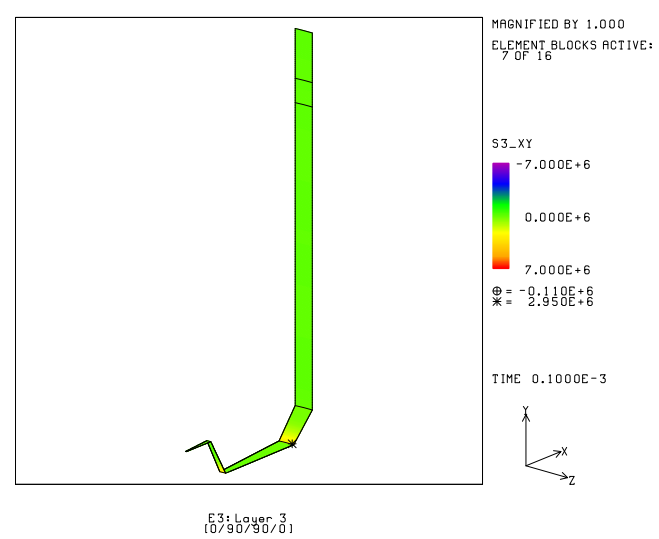

(b)

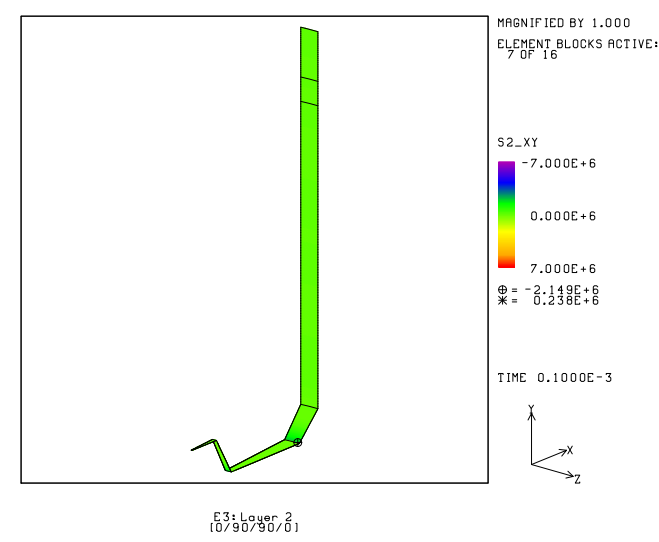

(d)

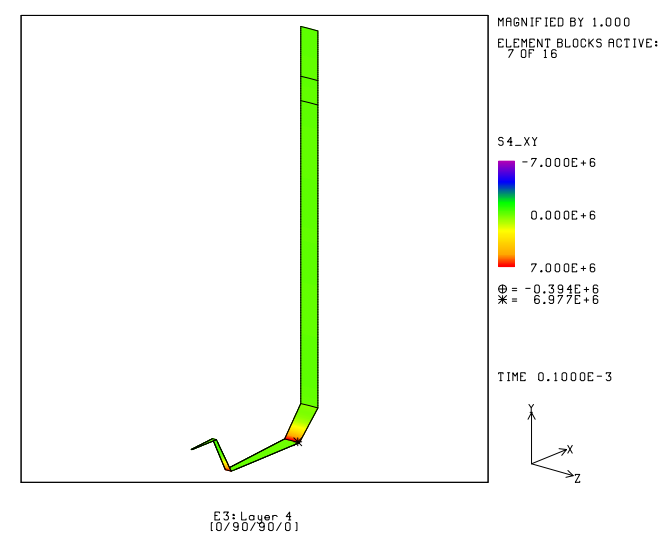

Figure 4.18: Radial-axial shear stresses due to internal pressurization for the [0/90/90/0] container modeled using primarily shell elements: (a) layer 1 (outermost layer); (b) layer 2; (c) layer 3; (d) layer 4 (innermost layer). 
(a)

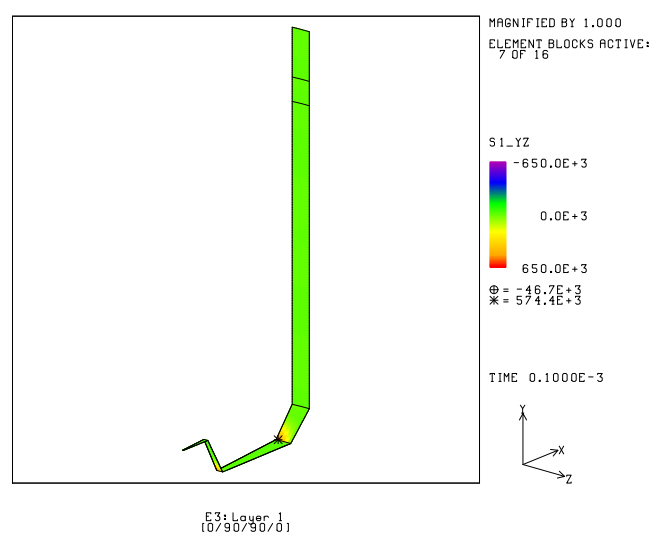

(c)

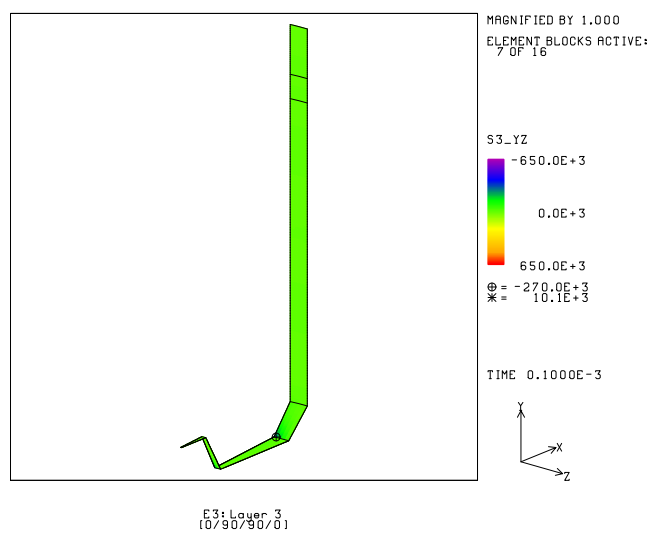

(b)

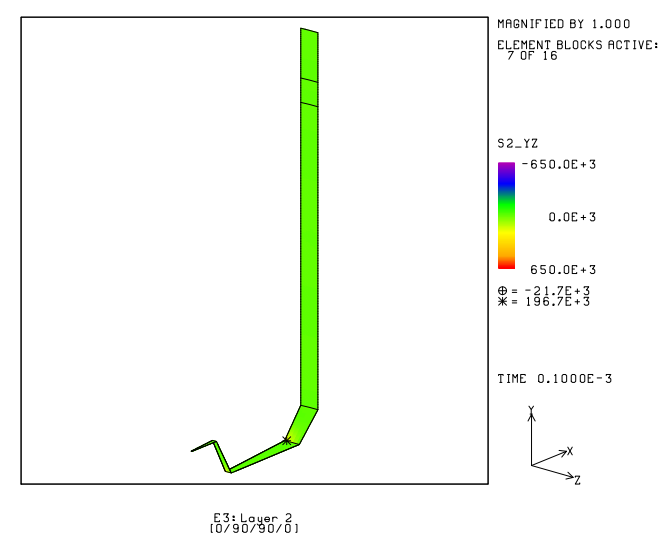

(d)

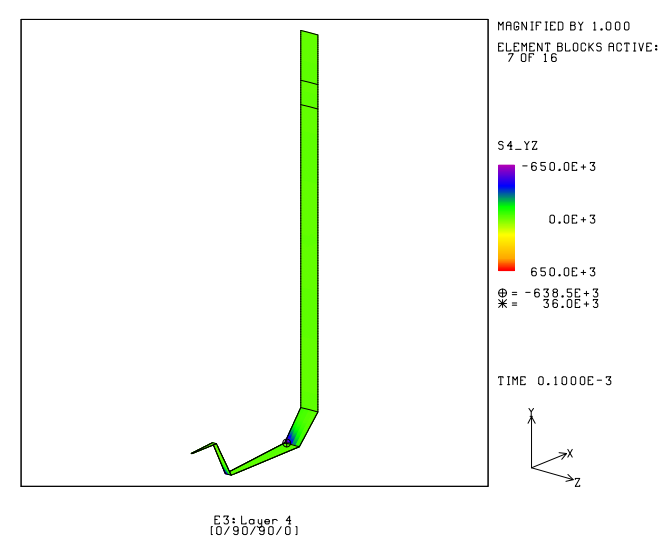

Figure 4.19: Negative of axial-circumferential shear stresses due to internal pressurization for the $[0 / 90 / 90 / 0]$ container modeled using primarily shell elements: (a) layer 1 (outermost layer); (b) layer 2; (c) layer 3; (d) layer 4 (innermost layer). 
(a)

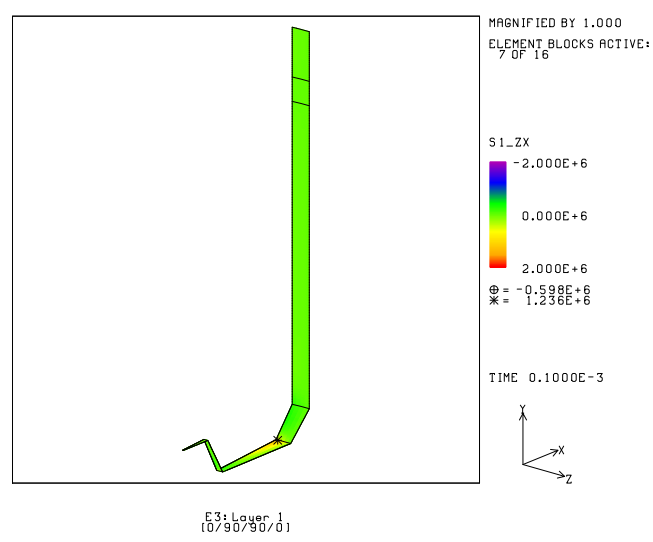

(c)

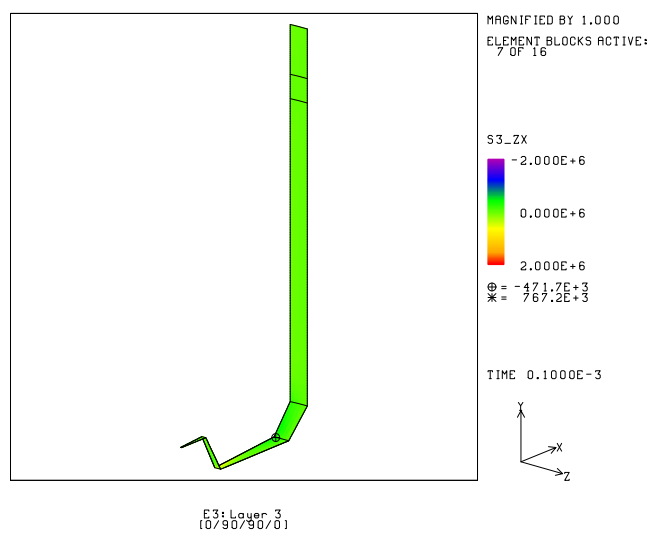

(b)

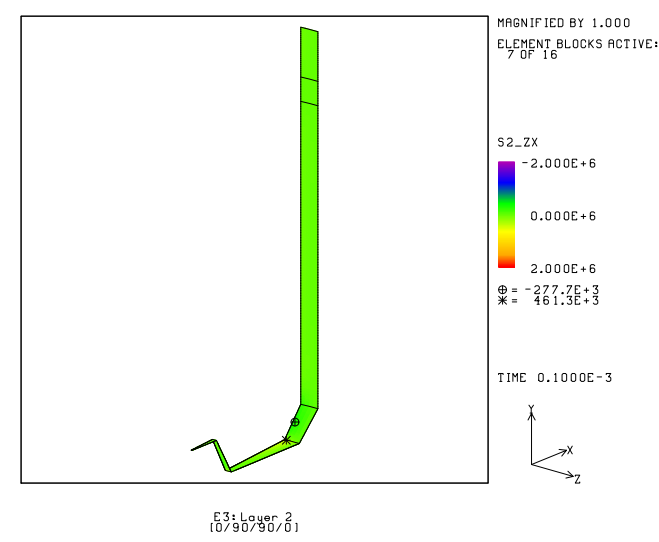

(d)

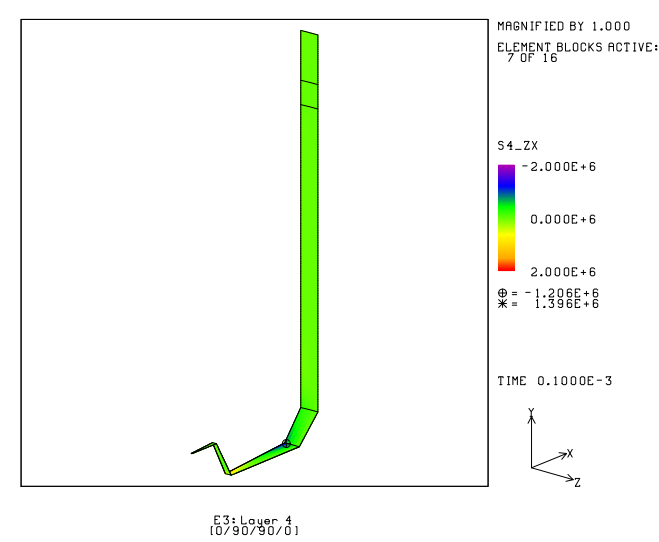

Figure 4.20: Negative of circumferential-radial shear stresses due to internal pressurization for the $[0 / 90 / 90 / 0]$ container modeled using primarily shell elements: (a) layer 1 (outermost layer); (b) layer 2; (c) layer 3; (d) layer 4 (innermost layer). 


\section{Comparison of Shell and Continuum Element Approaches for Composite Modeling}

In many cases of finite element analysis, using shell elements to model composite structures is more convenient and efficient compared to using continuum elements. First of all, in the model creation stage, it is easier to create meshes where a single shell element is used in the thickness direction to represent a laminated structure than to have to create separate element blocks for each layer as is required for a continuum approach. However, the specification of the resulting material model for the shell case requires careful attention in the case of non-symmetric laminates for which the sign of the entries in the extensional-bending coupling stiffness matrix $[B]$ is tied to the direction of the shell normal. That is, in such cases, it is necessary to compute $[B]$ with the stacking sequence specified with the top layer corresponding to the side of shell element defined by the positive element normal. Recall from basic composite theory that the signs of entries in the extensional stiffness matrix $[A]$ and the bending stiffness matrix $[D]$ do not depend on which side of the laminate is specified as the top for nonsymmetric lay-ups. The same holds true for the transverse shear stiffness matrix $\left[A_{t s}\right]$. If the stacking sequence used in computing $[B]$ is inadvertently the reverse of what it should be (e.g., [10/20/30/40] instead of [40/30/20/10]), the magnitudes of the entries in $[B]$ will be correct, but the signs will be the opposite of what is required. Such a mistake will likely lead to erroneous results for the integrated force and force-couple resultants in addition to the layerwise stresses, depending on the exact load applied and the non-symmetric stacking sequence being examined. Hence, care must be taken in such cases to match the sign of the entries in $[B]$ to the direction of the positive normal of shell elements in question. On the other hand, since the principal material directions are directly specified for each layer in the continuum approach, it is less likely that a mistake will be made in reversing the stacking sequence for the case of a non-symmetric laminate.

Another difference between the two approaches in ADAGIO/PRESTO concerns the results output. For the case where continuum elements are used, the layerwise stresses are directly available, but the integrated force and force-couple resultants must be calculated in a post-processing stage, if they are desired. In most cases, the layerwise 
stress distribution is of more interest. However, for the current shell element approach in ADAGIO/PRESTO, the reverse situation results where the integrated force and force-couple resultants are directly available and the layerwise stress distribution must be determined using ALGEBRA or another post-processing program. At present, an alternative layered shell approach is being implemented in ADAGIO/PRESTO where layerwise stresses will be directly available.

In some cases, the kinematic assumptions in the Mindlin shell theory used in ADAGIO are invalid for the problem at hand. It is quite possible that the normal to the laminate midplane in the undeformed configuration will not remain a straight line as the structure deforms. In most situations this will not be the case, but the user should always remain cognizant of the fact that Mindlin and other shell theories always incorporate more restrictive deformation assumptions than a general three-dimensional continuum theory. Whether the shell kinematic constraints are appropriate or not are, of course, problem dependent and sometimes not known a priori.

Another potential issue with using shell elements to represent a composite structure results from the fact that traditional shell elements are of zero thickness, regardless of the material model being employed. For the current input interface available for the composite shell model in ADAGIO, the $[A],\left[A_{t s}\right],[B]$, and $[D]$ matrices which correspond to pre-integrating the material response through the thickness direction are input directly. In many cases, the structure being analyzed is purely a single laminated composite structure for which the fact that traditional shell elements have zero thickness is not an issue. However, this may not be the case if a structure is being analyzed where a composite laminate is sandwiched between other materials, especially if continuum elements are used for those other materials. In such situation, using a zero thickness shell element will result in the surrounding materials having the incorrect thickness or wrong offset, either of which may or may not significantly affect the analysis results.

Next to accuracy of results and the time and effort required to create a model (i.e., the mesh and input deck generation), the biggest concern of an analyst is likely to be the computational effort required to achieve a solution. At a minimum, at least one hex element must be used per layer for the continuum modeling approach, whereas a single shell element can used through the thickness of a laminate. The shell and 
hex elements typically used in ADAGIO/PRESTO each have a total of 24 degrees-offreedom. Hence, for a multilayer composite, using a continuum element approach will result in a larger number of unknowns that need to be calculated. In some situations, it may be necessary to use even more than one hex element through the thickness of each composite layer. Exactly how many elements through the thickness of a layer are required is, of course, problem dependent. Thus, in many cases, the computational effort required for modeling composites using shell elements in ADAGIO is less than that required when hex/brick continuum elements are used. However, in some cases, ADAGIO has a much harder time converging, if at all, when shell elements are used.

Many problems of interest involve axisymmetric geometries. However, in analyzing these and other composite structures, analysts must be careful not to rely too much on intuition gained from isotropic analyses. For instance, in the case of an isotropic structure possessing axisymmetric geometry under axisymmetric loads, no circumferential displacements will occur. Rather, in such a case, the structure can only expand/contract along the radial and axial directions. Hence, such a situation can be modeled by considering only a small sector (pie cut) and applying boundary conditions on the circumferential edges specifying that no circumferential displacements occur. However, in the case of a laminated composite structure possessing axisymmetric geometry under axisymmetric loads, circumferential displacements may or may not occur depending on the laminate stacking sequence. Thus, when a small sector is modeled for a such composite structure, it is not correct to simply apply a condition of no circumferential displacements along the sector circumferential edges. Rather, true cyclic periodic boundary conditions must be used such that the sector modeled deforms in a way that the periodicity is preserved in the response. That is, the response is still axisymmetric in that it is independent of the angular position when expressed using an appropriate cylindrical coordinate system, even though it may involve circumferential displacements. For example, if a $10^{\circ}$ sector is modeled, the deformations must be such that taking 36 of these deformed $10^{\circ}$ sectors, the ends would still fit together to form a complete $360^{\circ}$ ring with no gaps.

In general, continuum finite element modeling of composite structures appears to be more robust than shell finite element modeling in terms of determining the laminated behavior accurately and in achieving a solution using ADAGIO. However, the 
price paid is that the model creation phase can be more time consuming for an analyst and the model solution phase can be more computationally expensive compared to a shell model which can achieve the same accuracy for many problems. Of course, many times a little bit of effort upfront can be used to create a model which is not needlessly larger than required. For instance, periodic boundary conditions can be used for axisymmetric structures. There is, however, a practical limit on how small such a sector can be in such a case. Regardless of whether continuum or shell elements are used, careful attention must be paid when creating and analyzing a composite model, as much of the intuition acquired from the analysis of isotropic structures cannot be used. 


\section{Summary}

There have been several significant accomplishments over the course of this ASC AD project that are aligned with the general goals of Advanced Deployment projects:

1. A coding error was uncovered and corrected in the "nlve thermoset" model being employed in the ADAGIO computations.

2. For the first time, the isotropic nonlinear viscoelastic material model has been run in parallel using the SIERRA code.

3. A realistic DP application has been analyzed for a customer demonstrating new capabilities that are simply unavailable in commercial codes.

4. Additional flexibility was added to the orientation initialization routines of the orthotropic linear elastic model, based upon needs identified by the composite container analyses performed here.

5. The ability to analyze large composite problems using Sandia's SIERRA/ADAGIO code was successfully demonstrated. Previously, any such composite modeling at Sandia required the use of commercial codes which are limited in terms of problem size and in some cases by the unavailability of proper cyclic periodic boundary conditions.

6. A comparative study of modeling composites using continuum versus shell elements has been completed and the resulting general guidelines for analysts have been detailed herein. 


\section{$7 \quad$ References}

${ }^{1}$ Hammerand, D.C., Laminated Composites Modeling in ADAGIO/PRESTO, Sandia Report SAND2004-2143, Sandia National Laboratories, Albuquerque, NM, May 2004. 


\section{Appendix A: ADAGIO Input Deck for the Adhesive Joint Problem}

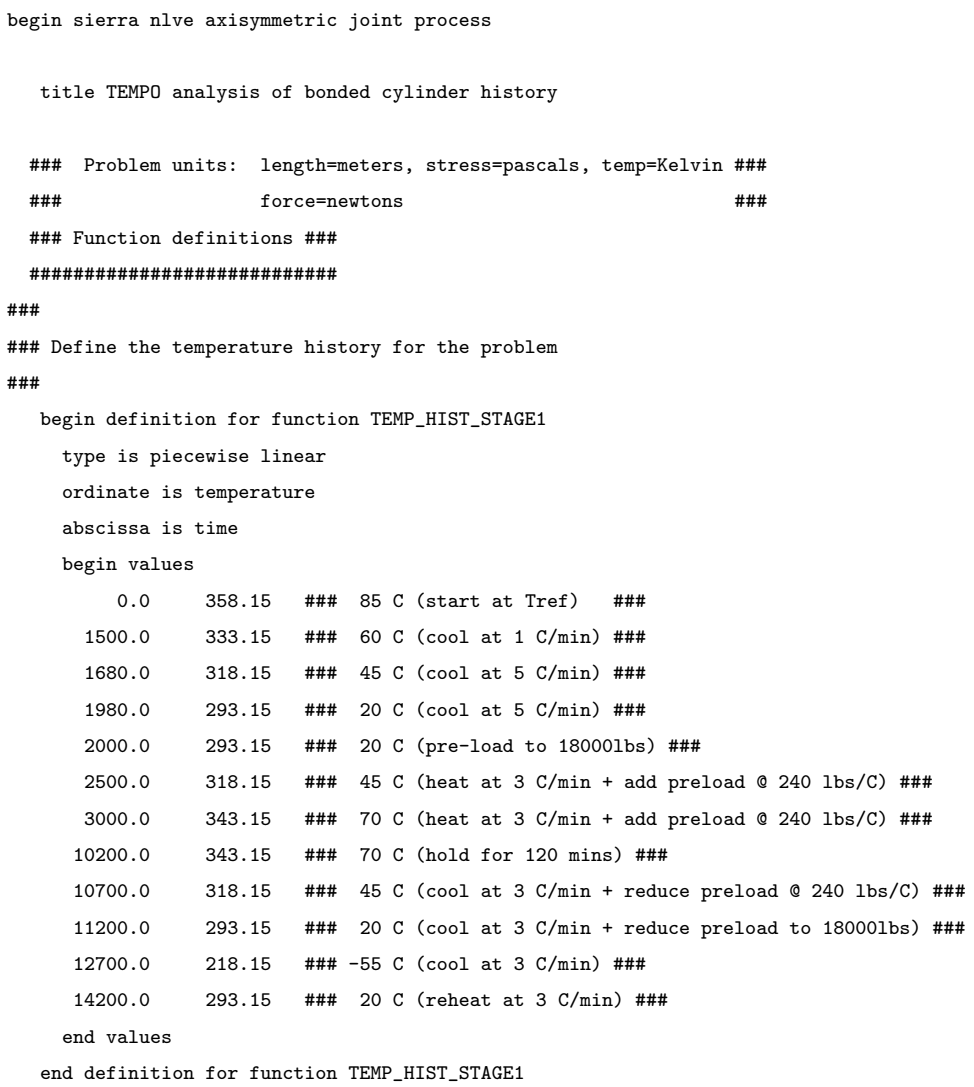




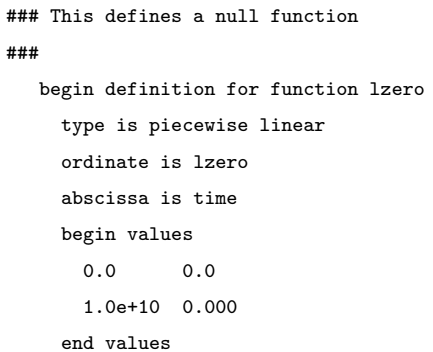

end definition for function lzero

\#\#\#

\#\#\# Define the polymer thermal strain as a function of temperature.

\#\#\# This is merely a place holder to populate a SIERRA input.

\#\#\# The nonlinear viscoelastic thermoset model is thermodynamically consistent

\#\#\# for finite strains.

\#\#\# In finite strain mechanics, there is no thermal strain definition.

\#\#\# Rather the constitutive equation predicts a pressure corresponding

\#\#\# a temperature change. SIERRA wants something anyway so here it is.

begin definition for function ADH_THERMAL_STRAIN

type is piecewise linear

ordinate is strain

abscissa is temperature

begin values

$\begin{array}{rr}-1000.0 & 0.0\end{array}$

$0.0 \quad 0.0$

$1000.0 \quad 0.0$

end values

end definition for function ADH_THERMAL_STRAIN

\#\#\#

\#\#\# This is the typical thermal strain definition as a function of temperature

\#\#\# for the metal.

begin definition for function AL_THERMAL_STRAIN

type is piecewise linear

ordinate is strain

abscissa is temperature

begin values

$-100.0-0.00234$ \#\#\# 6061T6 Alum Linear CTE=2.34E-5/C \#\#\#

$500.0 \quad 0.0117$

end values

end definition for function AL_THERMAL_STRAIN

\#\#\# Direction definitions \#\#\#

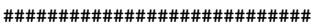

define direction $\mathrm{x}$ with vector $\begin{array}{llll}1.0 & 0.0 & 0.0\end{array}$

define direction $y$ with vector 0.01 .00 .0

define direction $\mathrm{z}$ with vector 0.00 .01 .0

\#\#\# Material definitions \#\#\#

\#\#\#\#\#\#\#\#\#\#\#\#\#\#\#\#\#\#\#\#\#\#\#\#\#\#

begin property specification for material ALuminum_6061T6

density $\quad=2710.0$

thermal strain function $=$ AL_THERMAL_STRAIN

begin parameters for model elastic

youngs modulus $=6.9 \mathrm{e}+10$

poissons ratio $=0.33$

end parameters for model elastic 
end property specification for material ALuminum_6061T6

begin property specification for material ALuminum_1100

$$
\text { density } \quad=2710.0
$$

thermal strain function $=$ AL_THERMAL_STRAIN

begin parameters for model elastic

youngs modulus $=6.9 \mathrm{e}+10$

poissons ratio $=0.33$

end parameters for model elastic

end property specification for material ALuminum_1100

begin property specification for material ALuminum_1100soft

density $\quad=2710.0$

thermal strain function $=$ AL_THERMAL_STRAIN

begin parameters for model elastic

youngs modulus $=1.0 \mathrm{E}+6$

poissons ratio $=0.33$

end parameters for model elastic

end property specification for material ALuminum_1100soft

begin property specification for material black_adhesive

$$
\text { density } \quad=1176.0
$$

thermal strain function $=$ ADH_THERMAL_STRAIN

begin parameters for model nlve_thermoset

bulk modulus $=6.0 \mathrm{e} 9$

shear modulus $=0.68 \mathrm{e} 9$

1 psi prony $1=0.0$

1 psi prony $2=0.0$

1 psi prony $3=0.0$

1psi prony $4=0.0$

1 psi prony $5=0.0$

1 psi prony $6=0.0$

1psi prony $7=0.0$

1 psi prony $8=0.0$

1 psi prony $9=0.0$

1 psi prony $10=0.0$

1 psi prony $11=0.0$

$1 \mathrm{psi}$ prony $12=0.0$

1 psi prony $13=0.0$

1 psi prony $14=0.0$

$1 \mathrm{psi}$ prony $15=0.0$

1psi prony $16=0.0$

1 psi prony $17=0.0$

$1 \mathrm{psi}$ prony $18=0.0$

1 psi prony $19=0.0$

1 psi prony $20=0.0$

$1 \mathrm{psi}$ prony $21=0.0$

$1 \mathrm{psi}$ prony $22=0.0$

1 psi prony $23=0.0$

$1 \mathrm{psi}$ prony $24=0.0$

$1 \mathrm{psi}$ prony $25=0.0$

1 psi prony $26=0.0$

$1 \mathrm{psi}$ prony $27=0.0$

1 psi prony $28=0.0$

1psi prony 29 $=0.0$

$1 \mathrm{psi}$ prony $30=0.0$

2 psi prony $1=0.0$ 
2 psi prony $2=0.0$ 2 psi prony $3=0.0$ 2 psi prony $4=0.0$ 2 psi prony $5=0.0$ 2 psi prony $6=0.0$ 2 psi prony $7=0.0$ 2 psi prony $8=0.0$ 2 psi prony $9=0.0$ 2 psi prony $10=0.0$ 2psi prony $11=0.0$ 2 psi prony $12=0.0$ 2 psi prony $13=0.0$ 2psi prony $14=0.0$ 2 psi prony $15=0.0$ 2psi prony $16=0.0$ 2 psi prony $17=0.0$ 2 psi prony $18=0.0$ 2psi prony $19=0.0$ 2 psi prony 20 $=0.0$ 2 psi prony $21=0.0$ 2 psi prony 22 $=0.0$ 2psi prony $23=0.0$ 2 psi prony $24=0.0$ 2psi prony 25 $=0.0$ 2 psi prony $26=0.0$ 2 psi prony $27=0.0$ $2 \mathrm{psi}$ prony $28=0.0$ 2psi prony 29 $=0.0$ 2 psi prony $30=0.0$ 3 psi prony $1=0.0$ 3psi prony $2=0.0$ 3psi prony $3=0.0$ 3 psi prony $4=0.0$ 3psi prony $5=0.0$ 3psi prony $6=0.0$ 3 psi prony $7=0.0$ 3psi prony $8=0.0$ 3psi prony $9=0.0$

3 psi prony $10=0.0$ 3 psi prony $11=0.0$ 3 psi prony $12=0.0$ 3 psi prony $13=0.0$ 3 psi prony $14=0.0$ 3psi prony $15=0.0$ 3 psi prony $16=0.0$ 3psi prony $17=0.0$ 3 psi prony $18=0.0$ 3 psi prony $19=0.0$ 3 psi prony 20 $=0.0$ 3psi prony $21=0.0$ 3 psi prony 22 $=0.0$ 3psi prony 23 $=0.0$ 3 psi prony $24=0.0$ 3psi prony 25 $=0.0$ 3psi prony 26 $=0.0$ 3 psi prony $27=0.0$ 3 psi prony 28 $=0.0$ 3psi prony 29 $=0.0$ 3psi prony $30=0.0$ 4psi prony $1=0.0$ 4 psi prony $2=0.0$ 
4 psi prony $3=0.0$

4 psi prony $4=0.0$

4 psi prony $5=0.0$

4psi prony $6=0.0$

4psi prony $7=0.0$

4 psi prony $8=0.0$

4 psi prony $9=0.0$

4 psi prony $10=0.0$

4 psi prony $11=0.0$

4 psi prony $12=0.0$

$4 \mathrm{psi}$ prony $13=0.0$

$4 \mathrm{psi}$ prony $14=0.0$

4psi prony $15=0.0$

4 psi prony $16=0.0$

$4 \mathrm{psi}$ prony $17=0.0$

4 psi prony $18=0.0$

4 psi prony $19=0.0$

4 psi prony 20 $=0.0$

4 psi prony $21=0.0$

4 psi prony $22=0.0$

$4 \mathrm{psi}$ prony $23=0.0$

4 psi prony $24=0.0$

4 psi prony $25=0.0$

$4 \mathrm{psi}$ prony $26=0.0$

4psi prony $27=0.0$

4 psi prony $28=0.0$

$4 \mathrm{psi}$ prony $29=0.0$

4psi prony $30=0.0$

1 psi ref $=6.4042 \mathrm{e}+05$

i1 deriv $1 \mathrm{psi}=0.0$

2 psi ref $=5.6998 \mathrm{e}+05$

$\mathrm{t}$ deriv $2 \mathrm{psi}=-1.2755 \mathrm{e}+03$

3 psi ref $=1.1684 \mathrm{e}+03$

$\mathrm{t}$ deriv $3 p s i=-3.1239 \mathrm{e}+00$

4 psi ref $=7.2275 \mathrm{e}-01$

$\mathrm{t}$ deriv $4 \mathrm{psi}=6.9662 \mathrm{e}-04$

$\mathrm{t} 2$ deriv $4 \mathrm{psi}=4.3610 \mathrm{e}-06$

reference temp $=358.15$

reference density $=1176$

wlf $\mathrm{c} 1=10$

wlf $\mathrm{c} 2=67$

b shift constant $=3.0706 \mathrm{e}+05$

shift ref value $=3.1390 \mathrm{e}+04$

wwbeta $1 \mathrm{psi}=0.18$

wwtau $1 \mathrm{psi}=5$

wwbeta $2 \mathrm{psi}=0.18$

wwtau 2psi $=0.00015$

wwbeta $3 \mathrm{psi}=0.18$

wwtau 3psi $=5$

wwbeta $4 \mathrm{psi}=0.15$

wwtau 4 psi $=2$

double integ factor $=0$

rubbery bulk $\bmod =4800000000$

i1 deriv r_bulk $=0$

glassy bulk mod $=6000000000$

i1 deriv g_bulk $=0$

rubbery shear $\bmod =9700000$

$t$ deriv $r_{-}$shear $=0$

i2 deriv $r_{-}$shear $=0$

glassy shear $\bmod =680000000$

$t$ deriv g_shear $=-1500000$ 
rubbery vol cte $=0.00053$

$\mathrm{t}$ deriv $r_{-} c t e=1 \mathrm{e}-7$

glassy vol cte $=0.000195$

$\mathrm{t}$ deriv g_cte $=0$

rubbery hcapacity $=3020$

$t$ deriv r_hcapacity $=3$

glassy hcapacity $=2420$

$t$ deriv g_hcapacity $=3$

GLASS TRANSITION TEM $=358.15$

PSI EQ 2I $=4.0761 \mathrm{e}+06$

PSI EQ IT $=-2.1633 e+03$

PSI EQ $2 \mathrm{~T}=-7.2857 \mathrm{e}+00$

PSI EQ $2 \mathrm{H}=8.2483 \mathrm{e}+03$

PSI EQ $3 I=0.0$

PSI EQ 2IT $=-9000.0$

PSI EQ I2T $=1.0284 \mathrm{e}+01$

PSI EQ $3 \mathrm{~T}=7.6802 \mathrm{e}-03$

PSI EQ 2HT $=0.0$

PSI EQ 4I $=0.0$

PSI EQ 3IT $=0.0$

PSI EQ 2I2T $=0.0$

PSI EQ I3T $=0.0$

PSI EQ $4 \mathrm{~T}=-8.7074 \mathrm{e}-05$

PSI EQ $4 \mathrm{H}=0.0$

PSI POT IT $=-1.1684 \mathrm{e}+03$

PSI POT $2 \mathrm{~T}=-7.2275 \mathrm{e}-01$

PSI POT 2IT $=0.0$

PSI POT I2T $=6.2479 \mathrm{e}+00$

PSI POT $3 \mathrm{~T}=-2.0899 \mathrm{e}-03$

PSI POT $4 \mathrm{~T}=-2.6166 \mathrm{e}-05$

relax time $1=1 \mathrm{e}-13$

relax time $2=1 \mathrm{e}-12$

relax time $3=1 \mathrm{e}-11$

relax time $4=1 \mathrm{e}-10$

relax time $5=1 \mathrm{e}-09$

relax time $6=1 \mathrm{e}-08$

relax time $7=1 \mathrm{e}-07$

relax time $8=1 \mathrm{e}-06$

relax time $9=3.16 \mathrm{e}-06$

relax time $10=1.0 \mathrm{e}-05$

relax time $11=3.16 \mathrm{e}-05$

relax time $12=1.0 \mathrm{e}-04$

relax time $13=3.16 \mathrm{e}-04$

relax time $14=1.0 \mathrm{e}-03$

relax time $15=3.16 \mathrm{e}-03$

relax time $16=1.0 \mathrm{e}-02$

relax time $17=3.16 \mathrm{e}-02$

relax time $18=1.0 \mathrm{e}-01$

relax time $19=3.16 \mathrm{e}-01$

relax time $20=1.0$

relax time $21=3.16$

relax time $22=10.0$

relax time $23=31.6$

relax time $24=100$

relax time $25=316$

relax time $26=1000$

relax time $27=3160$

relax time $28=10000$

relax time $29=31600$

relax time $30=100000$

end parameters for model nlve_thermoset 
end property specification for material black_adhesive

\#\#\# BV Problem definitions \#\#\#

\#\#\#\#\#\#\#\#\#\#\#\#\#\#\#\#\#\#\#\#\#\#\#\#\#\#

begin finite element model loading.

Database Name $=$ axicy19045.g

Database Type $=$ exodusII

begin parameters for block block_1 material ALuminum_6061T6

solid mechanics use model elastic

end parameters for block block_1

begin parameters for block block_2

material black_adhesive

solid mechanics use model nlve_thermoset

end parameters for block block_2

begin parameters for block block_3

material ALuminum_6061T6

solid mechanics use model elastic

end parameters for block block_3

begin parameters for block block_ 5 material ALuminum_1100soft

solid mechanics use model elastic

end parameters for block block_5

end finite element model loading1

begin finite element model loading2

Database Name $=$ axicy19045.g

Database Type $=$ exodusII

begin parameters for block block_1

material ALuminum_6061T6

solid mechanics use model elastic

end parameters for block block_1

begin parameters for block block_2

material black_adhesive

solid mechanics use model nlve_thermoset

end parameters for block block_2

begin parameters for block block_3

material ALuminum_6061T6

solid mechanics use model elastic

end parameters for block block_3

begin parameters for block block_ 5 material ALuminum_1100

solid mechanics use model elastic

end parameters for block block_ 5

end finite element model loading2 


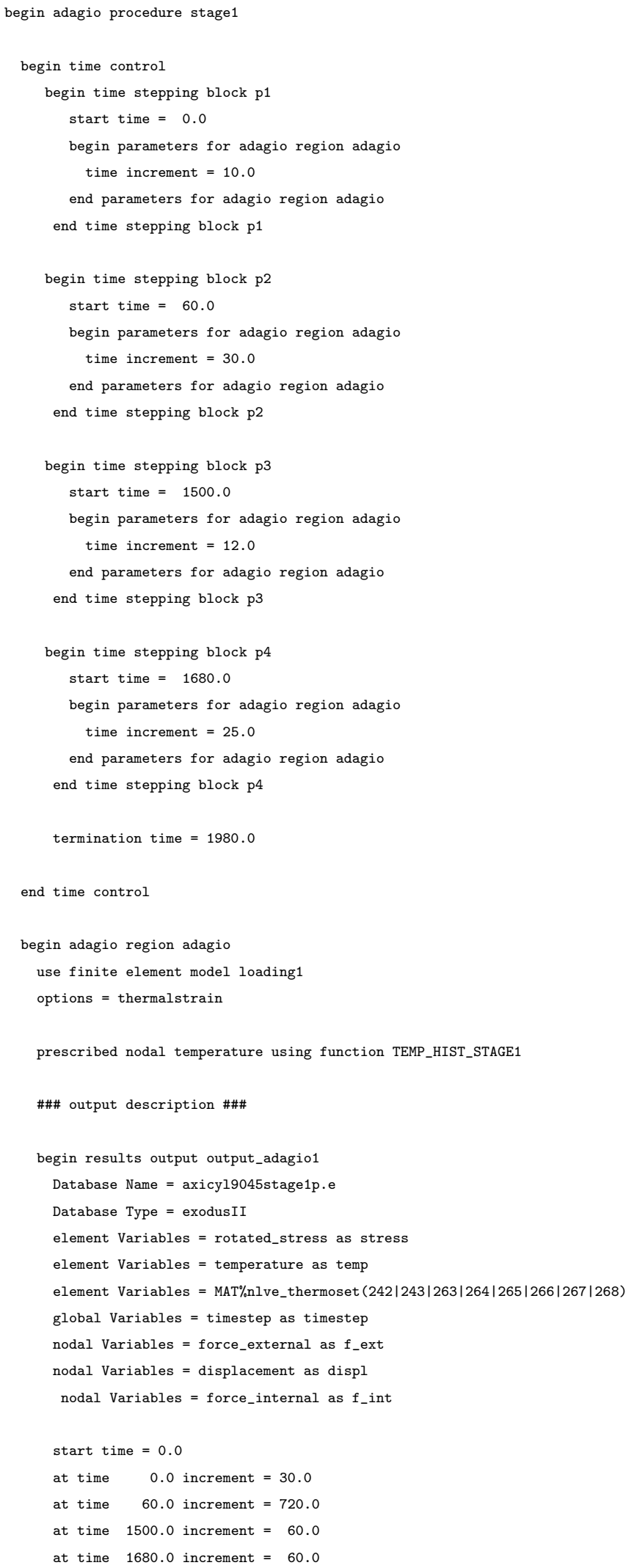


at time 1980.0 increment $=2.0$

termination time $=1980.0$

end results output output_adagio1

\#\#\# definition of $\mathrm{BCs} \# \# \#$

begin prescribed displacement

node set $=$ nodelist_20

direction $=z$

function $=$ lzero

scale factor $=0.0$

end prescribed displacement

begin prescribed displacement

node set $=$ nodelist_21

direction $=\mathrm{x}$

function $=$ lzero

scale factor $=0.0$

end prescribed displacement

begin prescribed displacement

node set $=$ nodelist_ 8

direction $=\mathrm{y}$

function $=$ lzero

scale factor $=0.0$

end prescribed displacement

\#\#\#\# Contacts \#\#\#\#\#

begin contact definition jstick 1

enforcement $=$ tied

contact surface surf_14 contains surface_14

contact surface surf_15 contains surface_15

begin interaction

master $=$ surf_14

slave $=$ surf_15

normal tolerance $=1.0 \mathrm{e}-5$

tangential tolerance $=1.0 \mathrm{e}-5$

capture tolerance $=1.0 \mathrm{e}-7$

tension release $=1.0 \mathrm{E}+25$

end interaction

end contact definition jstick1

begin contact definition sstick1

enforcement $=$ tied

contact surface surf_16 contains surface_16

contact surface surf_17 contains surface_17

begin interaction

master $=$ surf_16

slave $=$ surf_17

normal tolerance $=1.0 \mathrm{e}-5$

tangential tolerance $=1.0 \mathrm{e}-5$

capture tolerance $=1.0 \mathrm{e}-7$

tension release $=1.0 \mathrm{E}+25$

end interaction 
end contact definition sstick1

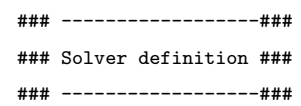

Loadstep predictor using line search type secant

begin adagio solver $\mathrm{cg}$

Target Relative Residual $=1.0 \mathrm{e}-6$

Target Residual $=0.008$ during $\mathrm{p} 1$

Target Residual $=0.008$ during $\mathrm{p} 2$

Target Residual $=0.008$ during $\mathrm{p} 3$

Target Residual $=0.008$ during $\mathrm{p} 4$

Maximum Iterations $=30000$

Minimum Iterations $=10$

Orthogonality measure for reset $=0.20$

Line Search type secant

iteration print $=50$

nodal preconditioning type $=$ elastic

end adagio solver cg

predictor scale factor $=1.0$ during $\mathrm{p} 1$

predictor scale factor $=1.0$ during $\mathrm{p} 2$

predictor scale factor $=1.0$ during $\mathrm{p} 3$

predictor scale factor $=1.0$ during $\mathrm{p} 4$

end adagio region adagio

end adagio procedure stage1

\#\#\#\#\#\#\#\#\#\#\#\#\#\#\#\#\#\#\#\#\#\#\#\#\#\#\#\#\#\#\#\#\#\#\#\#\#\#\#\#\#\#\#\#\#\#\#\#\#\#\#\#\#\#\#\#\#\#\#\#\#\#\#\#\#\#\#\#\#

begin adagio procedure stage 2

begin procedural transfer AgioToAgio

include variables from region adagio in block block_1

include variables from region adagio in block block_2

include variables from region adagio in block block_3

include variables from region adagio in block block_5

end procedural transfer AgioToAgio

begin time control

begin time stepping block p5

start time $=1980.0$

begin parameters for adagio region adagio

time increment $=0.1$

end parameters for adagio region adagio

end time stepping block p5

begin time stepping block p6

start time $=1981.0$

begin parameters for adagio region adagio

time increment $=0.25$

end parameters for adagio region adagio

end time stepping block $\mathrm{p} 6$

begin time stepping block p7

start time $=2000.0$

begin parameters for adagio region adagio 


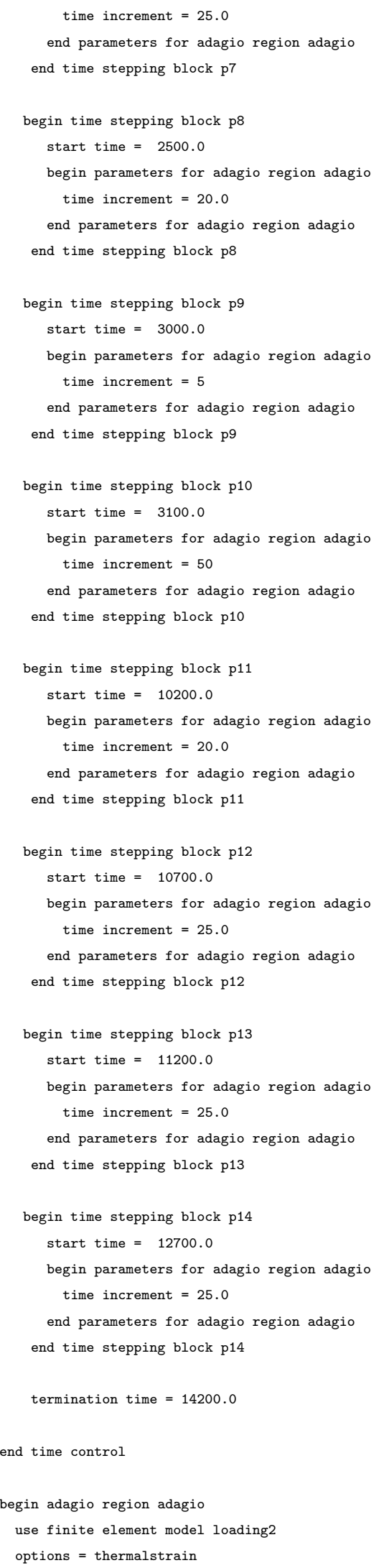


prescribed nodal temperature using function TEMP_HIST_STAGE1

\#\#\# output description \#\#\#

begin results output output_adagio2

Database Name $=$ axicy19045stage2.e

Database Type $=$ exodusII

element Variables $=$ rotated_stress as stress

element Variables $=$ temperature as temp

element Variables $=$ MAT\%nlve_thermoset $(242|243| 263|264| 265|266| 267 \mid 268)$

global Variables $=$ timestep as timestep

nodal Variables $=$ force_external as $f_{-}$ext

nodal Variables $=$ displacement as displ

$\#$

nodal Variables $=$ force_internal as $f_{-}$int

start time $=1980.0$

at time 1980.0 increment $=2.0$

at time 2000.0 increment $=100.0$

at time 2500.0 increment $=100.0$

at time 3000.0 increment $=1000.0$

at time 10200.0 increment $=100.0$

at time 10700.0 increment $=100.0$

at time 11200.0 increment $=300.0$

at time 12700.0 increment $=300.0$

termination time $=14200.0$

end results output output_adagio2

\#\#\# definition of $\mathrm{BCs} \# \# \#$

begin prescribed displacement

node set $=$ nodelist_20

direction $=z$

function $=$ lzero

scale factor $=0.0$

end prescribed displacement

begin prescribed displacement

node set $=$ nodelist_21

direction $=\mathrm{x}$

function $=$ lzero

scale factor $=0.0$

end prescribed displacement

begin prescribed displacement

node set $=$ nodelist_ 8

direction $=\mathrm{y}$

function = lzero

scale factor $=0.0$

end prescribed displacement

begin pressure

surface $=$ surface $\_13$

function $=$ apress

scale factor $=-1.0$

end pressure

\#\#\#\# Contacts \#\#\#\#\#\#

begin contact definition jstick2 


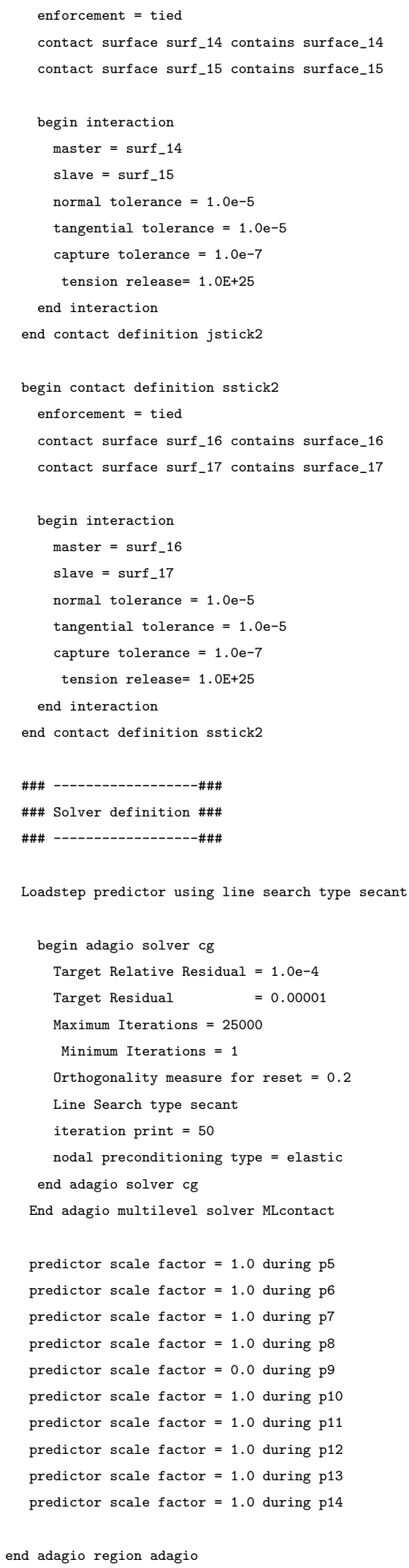


end adagio procedure stage 2

end sierra nlve axisymmetric joint process 


\section{Appendix B: ADAGIO Input Deck for $[30 / 60 / 50 / 10]$ Ring B Modeled Using Continuum Elements}

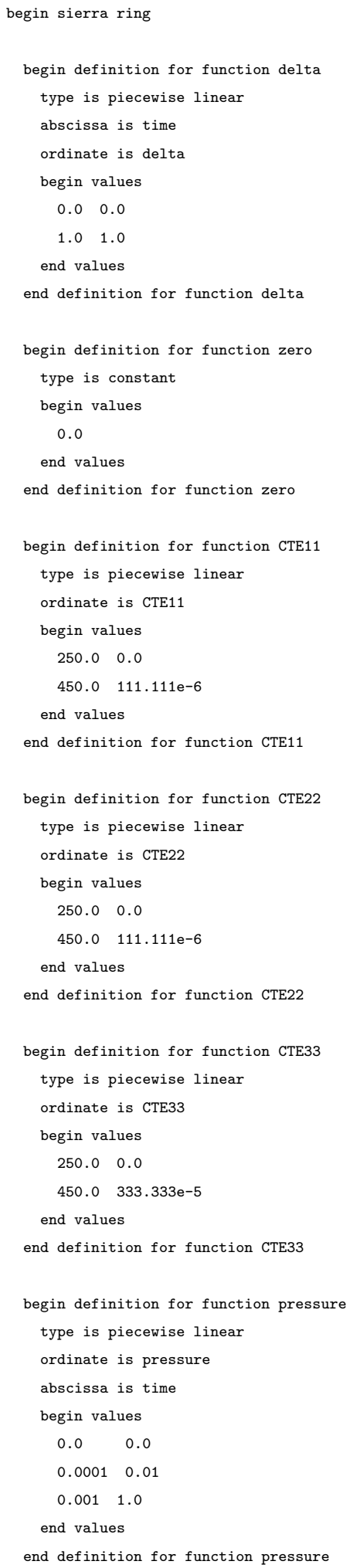




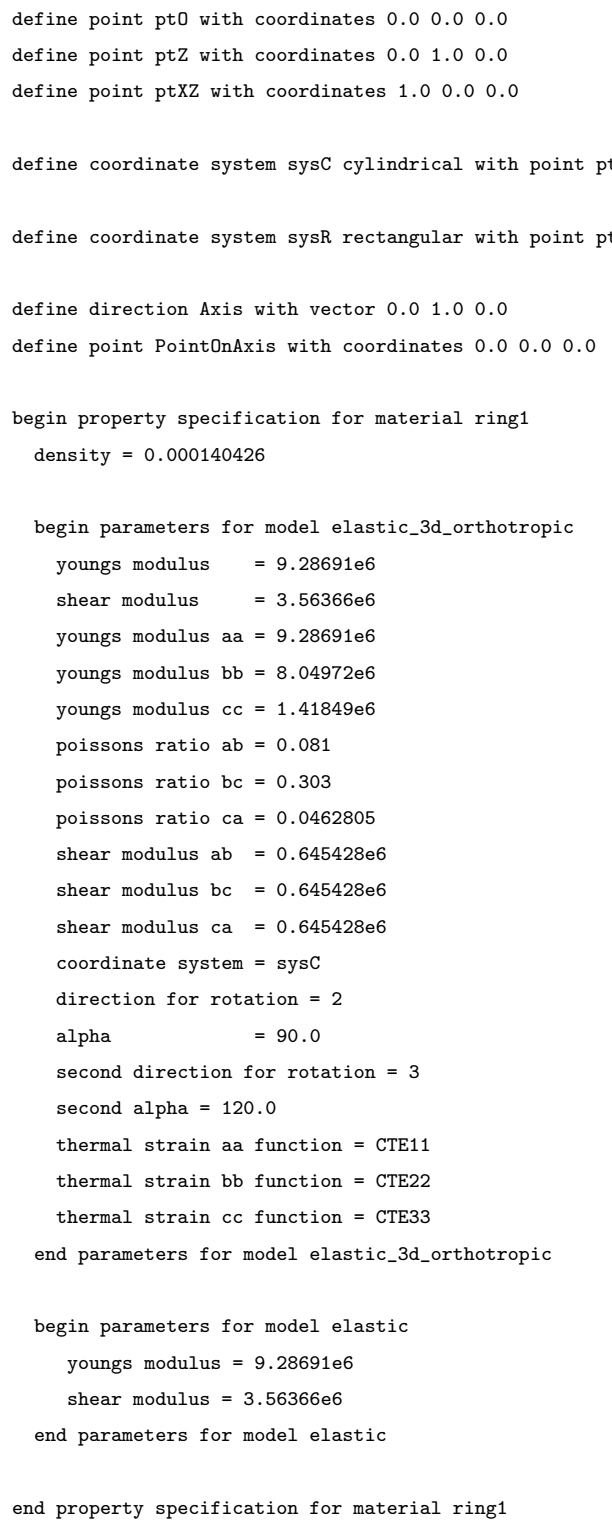

begin property specification for material ring2 density $=0.000140426$

begin parameters for model elastic_3d_orthotropic

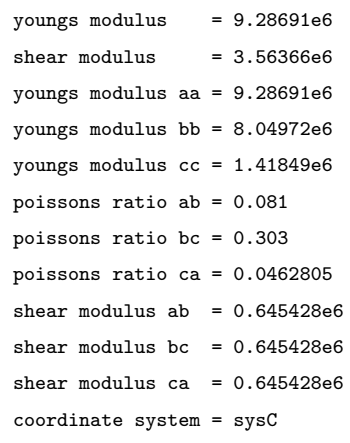




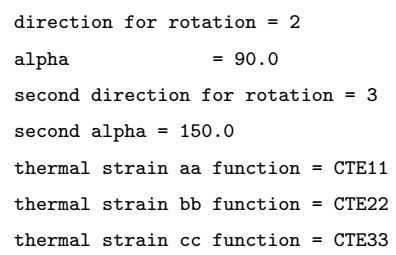

end property specification for material ring2

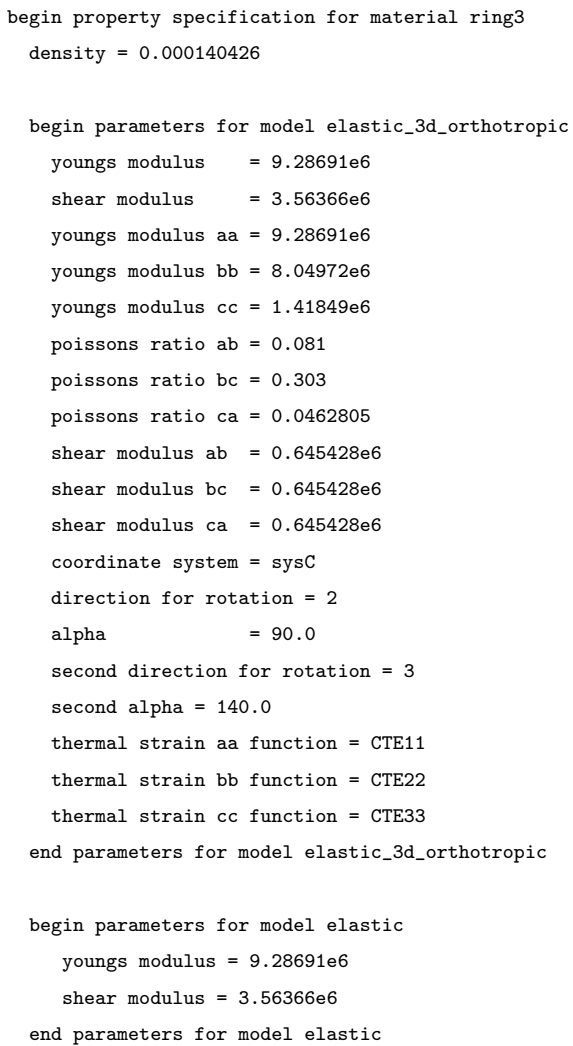


number of time steps $=10$

end parameters for adagio region contain_region

end time stepping block $\mathrm{p} 1$

termination time $=1 \cdot \mathrm{e}-3$

end time control

begin adagio region contain_region

use finite element model mesh1

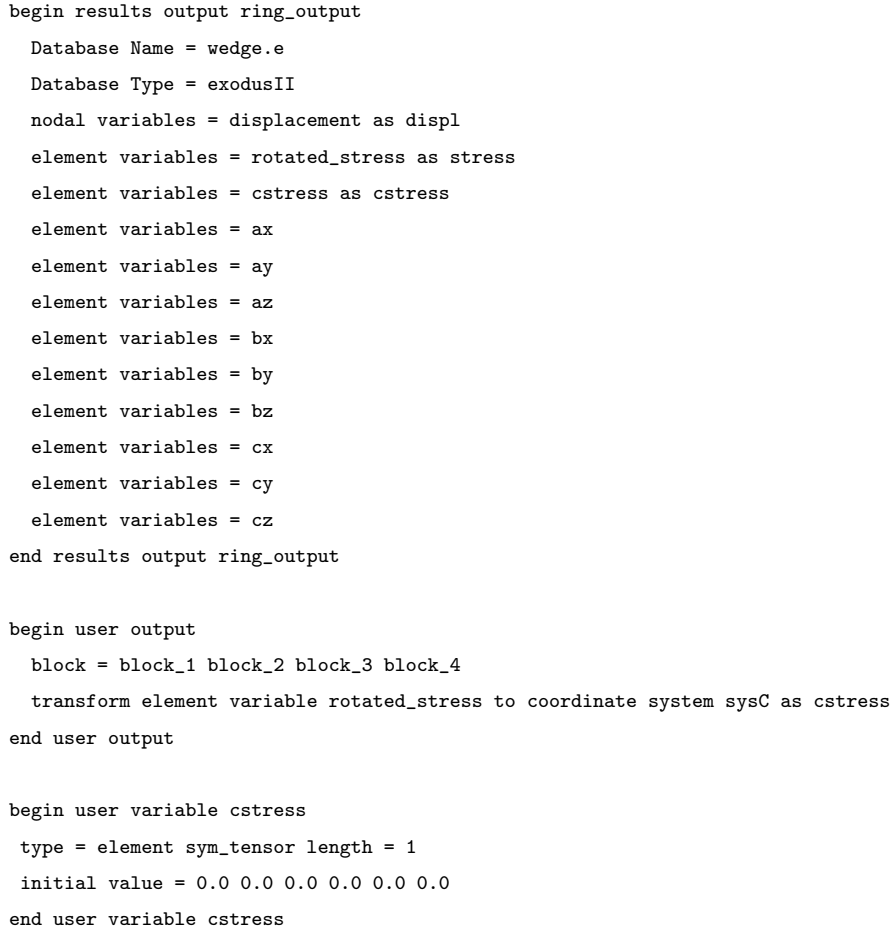




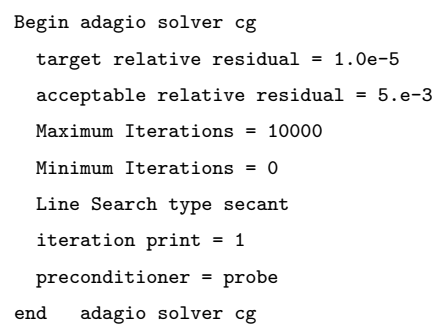

end adagio region contain_region

end adagio procedure load

end sierra ring 


\section{Appendix C: ADAGIO Input Deck for [30/60/50/10] Ring B Modeled Using Shell Elements}

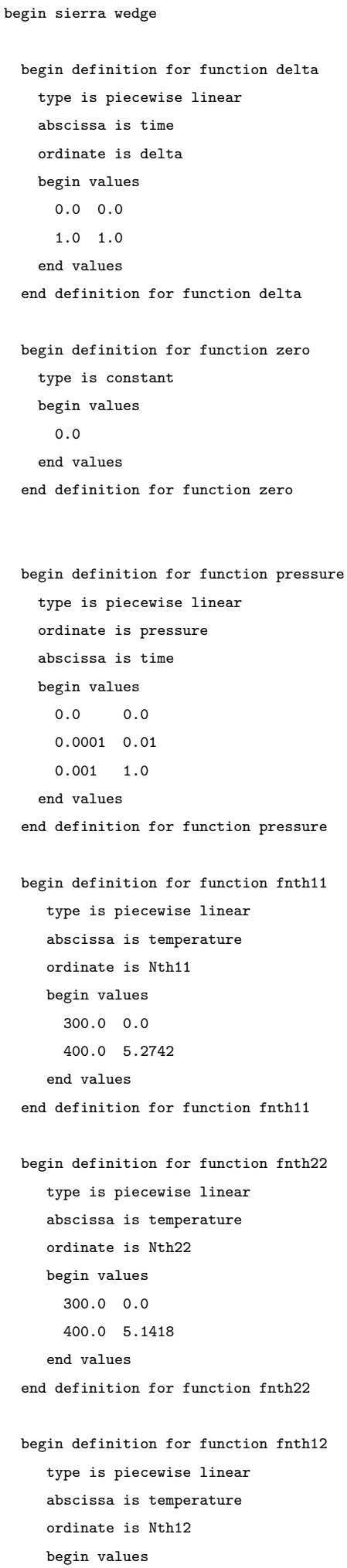




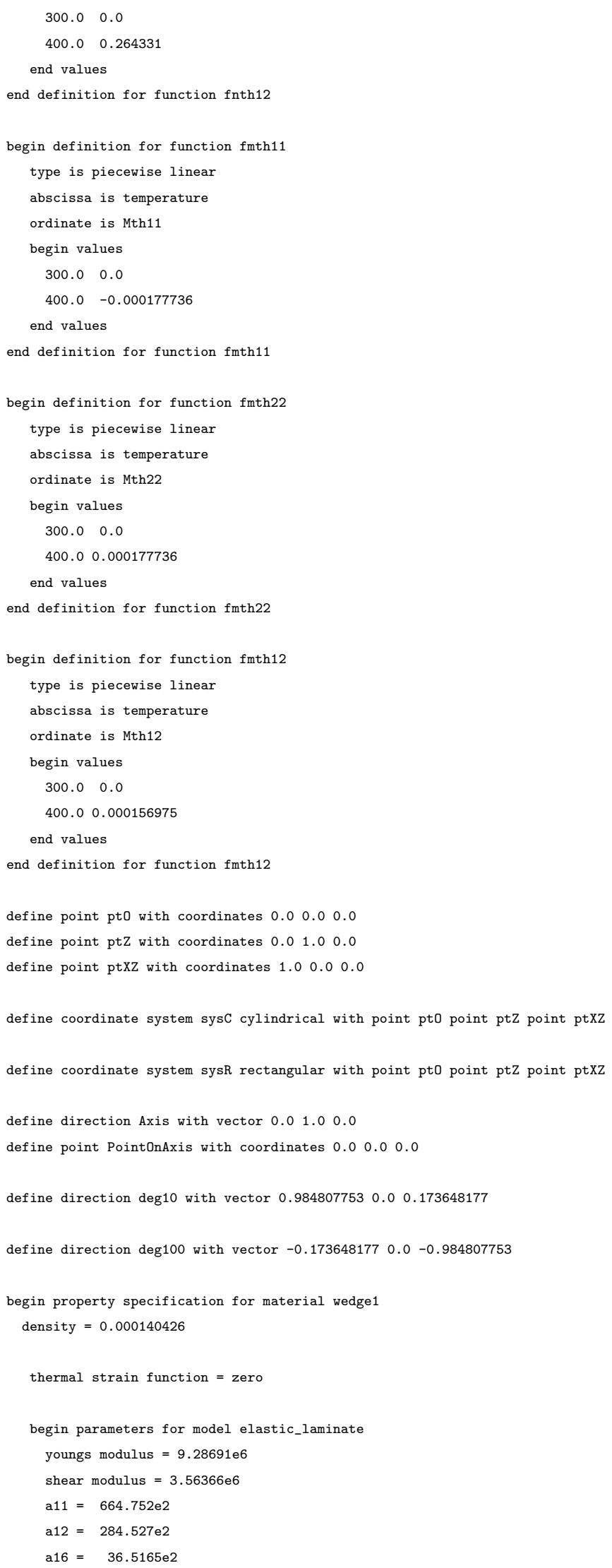


end parameters for adagio region contain_region

end time stepping block $\mathrm{p} 1$

termination time $=1 \cdot \mathrm{e}-3$

end time control

begin adagio region contain_region

use finite element model mesh1

begin results output wedge_output

Database Name $=$ wedge.e

Database Type $=$ exodusII

nodal variables $=$ displacement as displ

element Variables $=$ memb_stress as stress_memb

element Variables = top_stress as stress_top

element Variables $=$ bottom_stress as stress_bot

element Variables $=\mathrm{cm}_{-}$stress

element Variables $=c t_{-}$stress

element Variables $=$ cb_stress

element Variables $=$ element_thickness as thick

element Variables = element_area as area

element Variables $=$ axis1_dir as axis1

element Variables $=$ axis2_dir as axis2

end results output wedge_output

begin user output

block $=$ block_1

transform element variable memb_stress to coordinate system sysC as $\mathrm{cm}_{-}$stress

end user output

begin user variable $\mathrm{cm}_{-}$stress

type $=$ element sym_tensor length $=1$

initial value $=0.0 \quad 0.0 \quad 0.0 \quad 0.0 \quad 0.0 \quad 0.0$

end user variable $\mathrm{cm}_{-}$stress

begin user output

block $=$ block_1

transform element variable top_stress to coordinate system sysC as ct_stress

end user output

begin user variable ct_stress

type $=$ element sym_tensor length $=1$

initial value $=0.0 \quad 0.0 \quad 0.0 \quad 0.0 \quad 0.0 \quad 0.0$

end user variable ct_stress

begin user output

block $=$ block_ 1

transform element variable bottom_stress to coordinate system sysC as cb_stress

end user output

begin user variable cb_stress

type $=$ element sym_tensor length $=1$

initial value $=\begin{array}{llllll}0.0 & 0.0 & 0.0 & 0.0 & 0.0 & 0.0\end{array}$

end user variable cb_stress

\# inside surface: pressure loading

begin pressure

surface $=$ surface_100 


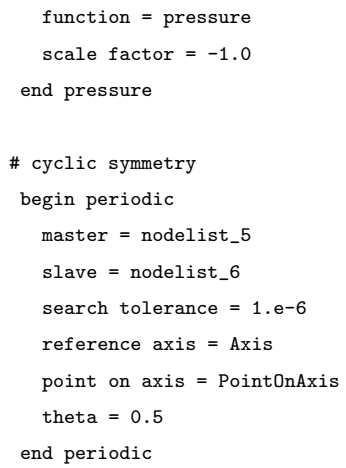




\section{Distribution:}

\begin{tabular}{|c|c|c|}
\hline 1 & 0196 & Rouse, Jerry, 1524 \\
\hline L & 0372 & Bishop, Joseph E., 1527 \\
\hline 1 & 0372 & Breivik, Nicole L., 1526 \\
\hline 1 & 0372 & Dempsey, Frank, 1526 \\
\hline 1 & 0372 & Dion, Kristin, 1526 \\
\hline 1 & 0372 & Duong, Henry, 1527 \\
\hline 1 & 0372 & Gruda, Jeffrey D., 1526 \\
\hline 1 & 0372 & Gwinn, Kenneth W., 1526 \\
\hline 1 & 0372 & Harding, David, 1526 \\
\hline 1 & 0372 & Hinnerichs, Terry D., 1526 \\
\hline 1 & 0372 & Holland, John, 1527 \\
\hline 1 & 0372 & Jones, Timothy C., 1526 \\
\hline 1 & 0372 & Jung, Joseph, 1527 \\
\hline 1 & 0372 & Kmetyk, Luba, 1527 \\
\hline 1 & 0372 & Longcope, Donald B., 1527 \\
\hline 1 & 0372 & May, Rodney A., 1526 \\
\hline 1 & 0372 & Metzinger, Kurt E., 1526 \\
\hline 1 & 0372 & Neidigk, Matthew, 1526 \\
\hline 1 & 0372 & Ostien, Jakob T., 1527 \\
\hline 1 & 0372 & Skousen, Troy J., 1527 \\
\hline 1 & 0372 & Wellman, Gerald W., 1527 \\
\hline 1 & 0376 & Adams, Charlie R., 1525 \\
\hline 1 & 0376 & Arguello, J. G. Lupe, 1526 \\
\hline 1 & 0376 & Rath, Jonathan S., 1527 \\
\hline 1 & 0376 & Stone, C. Mike, 1527 \\
\hline 1 & 0380 & Blanford, Mark L., 1542 \\
\hline 1 & 0380 & Crane, Nathan K., 1542 \\
\hline 1 & 0380 & Gilmartin, William, 1542 \\
\hline 1 & 0380 & Gullerud, Arne, 1542 \\
\hline 1 & 0380 & Hales, Jason D., 1542 \\
\hline
\end{tabular}




\begin{tabular}{|c|c|c|}
\hline 1 & 0380 & Heinstein, Martin W., 1542 \\
\hline 1 & 0380 & Key, Samuel W., 1542 \\
\hline 1 & 0380 & Koteras, J. Richard, 1542 \\
\hline 1 & 0380 & Perschbacher, Brent, 1542 \\
\hline 1 & 0380 & Pierson, Kendall H., 1542 \\
\hline 1 & 0380 & Porter, Vicki, 1542 \\
\hline 1 & 0380 & Reese, Garth M., 1542 \\
\hline 1 & 0380 & Spencer, Benjamin, 1542 \\
\hline 1 & 0380 & Walsh, Timothy F., 1542 \\
\hline 1 & 0380 & Wilson, Christopher Riley, 1542 \\
\hline 1 & 0553 & Bateman, Vesta I., 1526 \\
\hline 1 & 0553 & Smallwood, David O., 1524 \\
\hline 1 & 0555 & Garrett, Mark S., 1522 \\
\hline 1 & 0555 & Nelson, Curtis F., 1524 \\
\hline 1 & 0555 & Stasiunas, Eric C., 1524 \\
\hline 1 & 0557 & Baca, Thomas J., 1525 \\
\hline 1 & 0557 & C de Baca, John, 1527 \\
\hline 1 & 0557 & Cap, Jerome S., 1527 \\
\hline 1 & 0557 & Carne, Thomas G., 1524 \\
\hline 1 & 0557 & Dorrell, Larry, 1525 \\
\hline 1 & 0557 & Edwards, Timothy, 1527 \\
\hline 1 & 0557 & Epp, David S., 1525 \\
\hline 1 & 0557 & Griffith, D. Todd, 1524 \\
\hline 1 & 0557 & Martinez, Dominic, 1525 \\
\hline 1 & 0557 & Mayes, Randall L., 1525 \\
\hline 1 & 0557 & O'Gorman, Christian C., 1525 \\
\hline 1 & 0557 & Roberts, Nathaniel D., 1525 \\
\hline 1 & 0557 & Simmermacher, Todd W., 1524 \\
\hline 1 & 0615 & Roach, Dennis P., 6252 \\
\hline 1 & 0835 & Naething, Richard M., 1542 \\
\hline 1 & 0836 & Shumacher, Shane C., 1516 \\
\hline
\end{tabular}




\begin{tabular}{|c|c|c|}
\hline 1 & 0847 & Bitsie, Fernando, 1524 \\
\hline 1 & 0847 & Dohrmann, Clark, 1524 \\
\hline 1 & 0847 & Field, Richard V., Jr., 1524 \\
\hline 1 & 0847 & Holzmann, Wil A., 1525 \\
\hline 1 & 0847 & Miller, A. Keith, 1525 \\
\hline 1 & 0847 & Redmond, James M., 1524 \\
\hline 1 & 0847 & Rice, Amy E., 1525 \\
\hline 1 & 0847 & Segalman, Daniel J., 1524 \\
\hline 1 & 0847 & Simmons, Leslie, 1525 \\
\hline 1 & 0847 & Starr, Michael J., 1524 \\
\hline 1 & 0847 & Tipton, D. Gregory, 1525 \\
\hline 1 & 0847 & Walther, Howard P., 1527 \\
\hline 1 & 0847 & Wilson, Peter J., 1520 \\
\hline 1 & 0888 & Adolf, Douglas B., 1821 \\
\hline 1 & 0888 & Clough, Roger L., 1821 \\
\hline 1 & 0893 & Chambers, Robert S., 1523 \\
\hline 1 & 0893 & Chaplya, Pavel, 1523 \\
\hline 1 & 0893 & Cox, James V., 1523 \\
\hline 10 & 0893 & Hammerand, Daniel, 1523 \\
\hline 1 & 0893 & Lavin, Colby, 1523 \\
\hline 1 & 0893 & Lo, David, 1523 \\
\hline 1 & 0893 & Neilsen, Mike K., 1523 \\
\hline 1 & 0893 & Pott, John, 1523 \\
\hline 1 & 0893 & Reedy, E. Dave, 1523 \\
\hline 1 & 0893 & Scherzinger, William, 1523 \\
\hline 1 & 0893 & File Copy, 1523 \\
\hline 1 & 0902 & Allen, Matthew Scott, 1524 \\
\hline 1 & 0902 & Fulcher, Clay W. G., 1527 \\
\hline 1 & 1310 & Massad, Jordan E., 1524 \\
\hline 1 & 1310 & Sumali, Anton, 1524 \\
\hline
\end{tabular}




$\begin{array}{lll}6 & 9042 & \text { Brown, Arthur A., 8763 } \\ 1 & 9042 & \text { Chen, Er-Ping, 8770 } \\ 1 & 9042 & \text { Kwon, Davina M., 8774 } \\ 1 & 9042 & \text { Ohashi, Yuki, 8774 } \\ 1 & 9403 & \text { Foulk, James W., 8763 } \\ 1 & 9403 & \text { Shepodd, Timothy J., 8762 } \\ 1 & 0899 & \text { Technical Library, 9616 } \\ 1 & 9018 & \text { Central Technical Files, 8945-1 }\end{array}$

